\title{
Probability density function of SDEs with unbounded and path-dependent drift coefficient
}

\author{
Dai Taguchi * and Akihiro Tanaka $^{\dagger}$
}

October 9, 2019

\begin{abstract}
In this paper, we first prove that the existence of a solution of SDEs under the assumptions that the drift coefficient is of linear growth and path-dependent, and diffusion coefficient is bounded, uniformly elliptic and Hölder continuous. We apply Gaussian upper bound for a probability density function of a solution of SDE without drift coefficient and local Novikov condition, in order to use Maruyama-Girsanov transformation. The aim of this paper is to prove the existence with explicit representations (under linear/super-linear growth condition), Gaussian two-sided bound and Hölder continuity (under sub-linear growth condition) of a probability density function of a solution of SDEs with path-dependent drift coefficient. As an application of explicit representation, we provide the rate of convergence for an EulerMaruyama (type) approximation, and an unbiased simulation scheme.

2010 Mathematics Subject Classification: 65C30; 62G07; 35K08; 60H35

Keywords: Probability density function; Maruyama-Girsanov theorem; Gaussian two-sided bound; Parametrix method; Euler-Maruyama scheme; Unbiased simulation; Hölder continuity
\end{abstract}

\section{Contents}

1 Introduction

2 SDEs with unbounded and path-dependent drift

3 PDF of a solution of SDEs with unbounded and path-dependent drift

3.1 Existence and representations . . . . . . . . . . . . . . . . . 9 9

3.2 Gaussian two-sided bound and continuity of pdf . . . . . . . . . . . . . . 11

3.3 SDEs with bounded and path-dependent drift . . . . . . . . . . . . . 20

*Research Institute for Interdisciplinary Science, Okayama University 3-1-1 Tsushima-naka, Kita-ku Okayama 700-8530, Japan, email: dai.taguchi.dai@gmail.com

${ }^{\dagger}$ Graduate School of Engineering Science, Osaka University, 1-3, Machikaneyama-cho, Toyonaka, Osaka, 5608531, Japan / Sumitomo Mitsui Banking Corporation, 1-1-2, Marunouchi, Chiyoda-ku, Tokyo, 100-0005, Japan, Email: tnkaki2000@gmail.com 
3.4 Sharp bounds for a pdf of Brownian motion with bounded drift . . . . . . . . . . . 24

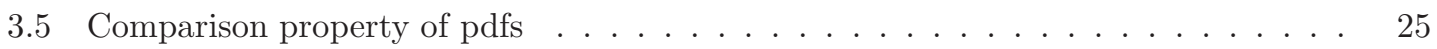

3.6 Application to an Euler-Maruyama type scheme . . . . . . . . . . . . 27

3.7 Markovian SDEs with unbounded drift . . . . . . . . . . . . . . . . . . . 30

3.8 Application to unbiased simulation scheme . . . . . . . . . . . . . . . 34

3.9 Hölder continuity of pdf . . . . . . . . . . . . . . . . . . . 35

4 One-dimensional SDEs with super-linear growth coefficients 38

5 Appendix 45

5.1 On some Beta type integral ........................... 45

\section{Introduction}

Let $X^{x}=\left(X_{t}^{x}\right)_{t \geq 0}$ be a solution of path-dependent $d$-dimensional stochastic differential equations (SDEs)

$$
\mathrm{d} X_{t}^{x}=b\left(t, X^{x}\right) \mathrm{d} t+\sigma\left(t, X_{t}^{x}\right) \mathrm{d} W_{t}, t \geq 0, X_{0}^{x}=x \in \mathbb{R}^{d},
$$

where $W=\left(W_{t}\right)_{t \geq 0}$ is a $d$-dimensional standard Brownian motion on a probability space $(\Omega, \mathcal{F}, \mathbb{P})$ with a filtration $\left(\mathcal{F}_{t}\right)_{t \geq 0}$ satisfying the usual conditions, and the drift coefficient $b:[0, \infty) \times$ $C\left([0, \infty) ; \mathbb{R}^{d}\right) \rightarrow \mathbb{R}^{d}$ and diffusion matrix $\sigma:[0, \infty) \times \mathbb{R}^{d} \rightarrow \mathbb{R}^{d \times d}$ are measurable.

The existence and regularity of a probability density function (pdf) of $X_{t}^{x}$ with respect to Lebesgue measure have been studied by many authors. If the drift $b:[0, \infty) \times \mathbb{R}^{d} \rightarrow \mathbb{R}^{d}$ is bounded Hölder continuous and diffusion matrix $\sigma$ is bounded, uniformly elliptic and Hölder continuous, then it is well-known that by using Levi's parametrix method (see, [15]), there exists the fundamental solution of parabolic type partial differential equations (Kolmogorov equation), and by FeynmanKac formula, it is a pdf of a solution of associated SDEs (see also, [35, 36, 39, 45]). Note that the parametrix method can be applied to the case of $L^{p}\left([0, T] \times \mathbb{R}^{d}\right)$-valued drift with $p \geq d+2$ [53], Hölder continuous (unbounded) drift [1], Brownian motion with signed measure belonging to the Kato class [29] and Hyperbolic Brownian motion with drift [26]. Moreover, Qian and Zheng [54, 55] provided a sharp two-sided bounds for a pdf of a Brownain motion with bounded drift coefficient by using pinned diffusion arguments (or regular conditional probability) and by choosing a parametrix as the pdf of bang-bang diffusion process. On the other hand, the existence, Gaussian two-sided bound and Hölder continuity for the fundamental solution of the parabolic equations in divergence form were proved by Aronson 2 and Nash [48. It is worth noting that Fabes and Kenig [13. provided an example of diffusion coefficient $\sigma$ such that the law of $X_{t}=x+\int_{0}^{t} \sigma\left(s, X_{s}\right) \mathrm{d} W_{s}$ is purely singular with respect to Lebesgue measure.

There are several methods to study a pdf as a probabilistic approach. One of the most useful tool is the Malliavin calculus (see, [27, 51, 61]). Kusuoka and Stroock [37] proved if the coefficients are Fréchet differentiable and diffusion coefficient uniformly elliptic, then a solution of path-dependent SDEs admits a smooth pdf. This results were extended by Takeuchi 63 , for the existence and smoothness of a joint pdf of finite dimensional distribution of SDEs. By using 
the Malliavin calculus, De Marco [10] studied a local existence of a pdf under local smoothness conditions on the coefficients, Kohatsu-Higa and Tanaka 32 studied the existence of a pdf of additive functionals of SDEs with bounded measurable drift coefficient, Hayashi, Kohatsu-Higa and Yûki [21, 22] studied the Hölder continuity, and recently, Olivera and Tudor [52] proved that by using Itô-Tanaka trick or Zvonkin transform (e.g. 65, 67]), a solution of SDE with Hölder continuous drift (unbounded) can be transformed by diffeomorphism to an equivalent equation with differentiable coefficients and a pdf. On the other hand, by using the stochastic control method, Sheu [60] study the Gaussian two-sided bound for a pdf of a solution of time-homogeneous Markovian SDEs $\mathrm{d} X_{t}=b\left(X_{t}\right) \mathrm{d} t+\sigma\left(X_{t}\right) \mathrm{d} W_{t}$.

The existence of a pdf of a solution of SDEs with path-dependents and non-smooth coefficients have been studied recently. Fournier and Printems 14 proved the existence of a pdf of a solution of one-dimensional SDEs with path-dependent coefficients, stochastic heat equations and Lévy driven SDEs, by using "one-step" Euler-Maruyama scheme and Fourier transform approach. As an extension of the approach in [14], Bally and Caramellino [3] provided an interpolation method, and proved the existence of a pdf for a solution of multi-dimensional path-dependent SDEs.

On the other hand as a perturbation approach, the parametrix method and MaruyamaGirsanov theorem are also useful tool in order to prove the existence of a pdf of a solution of SDEs with path-dependents coefficients. Frikha and $\mathrm{Li}$ [17] studied the existence of a weak solution of SDEs with path-dependent coefficients and its pdf by using the parametrix method. In the case of bounded and path-dependent drift coefficient, Makhlouf [42] and Kusuoka 38] studied the existence, explicit representation, Gaussian two-sided bound and Hölder continuity (see also [6, 7]). In particular, Makhlouf (Theorem 3.1 in [42]) showed that the following representation holds for a pdf, denoted by $p_{t}(x, \cdot)$, of Brownian motion with random drift $\mathrm{d} X_{t}=b_{t} \mathrm{~d} t+\mathrm{d} W_{t}, X_{0}=x$ :

$$
p_{t}(x, y)=g_{t}(x, y)+\int_{0}^{t} \mathbb{E}\left[\left\langle\nabla g_{t-s}\left(X_{s}, y\right), b_{s}\right\rangle\right] \mathrm{d} s, \text { a.e., } y \in \mathbb{R}^{d},
$$

where $g_{t}(x, y):=\frac{\exp \left(-|y-x|^{2} / 2 t\right)}{(2 \pi t)^{d / 2}}$, which is an analogue of the parametrix method. On the other hand, Kusuoka [38] showed that a pdf of a solution of path-dependent SDE (11) with bounded drift coefficient, denoted by $p_{t}(x, \cdot)$, has the following representation:

$$
p_{t}(x, y)=q(0, x ; t, y) \mathbb{E}\left[Z_{t}\left(1, Y^{0, x}\right) \mid Y_{t}^{0, x}=y\right] \text {, a.e., } y \in \mathbb{R}^{d},
$$

where $q(0, x ; t, \cdot)$ is the pdf of a solution of SDE without drift: $\mathrm{d} Y_{t}^{0, x}=\sigma\left(t, Y_{t}^{0, x}\right) \mathrm{d} W_{t}, Y_{0}^{0, x}=x$, $Z_{t}\left(1, Y^{0, x}\right)$ is the Girsanov density (see, Theorem 2.3 below), and $\mathbb{E}\left[\cdot \mid Y_{t}^{0, x}=y\right]$ is the expectation of a regular conditional probability given $Y_{t}^{0, x}=y$ for $y \in \mathbb{R}^{d}$. This representation is an analogue of Maruyama's result on the proof of Girsanov's theorem (see, Theorem 1 in 43 ). Note that these representations were also shown by Qian and Zheng [56] (see, Lemma 2.3 and Theorem 2.4 in [56]) for time-homogeneous diffusion processes with drift coefficient $b: \mathbb{R}^{d} \rightarrow \mathbb{R}^{d}$ satisfying at most linear growth and Novikov condition.

The aim of this paper is to prove that the existence of a weak solution of SDEs under the assumptions that the drift coefficient is of linear growth and path-dependent, and diffusion coefficient is bounded, uniformly elliptic and Hölder continuous. We apply the Gaussian upper bound for the pdf of a solution of SDE $\mathrm{d} Y_{t}^{0, x}=\sigma\left(t, Y_{t}^{0, x}\right) \mathrm{d} W_{t}, Y_{0}^{0, x}=x$ and "local" Novikov condition, in order to use Maruyama-Girsanov transformation. We will also show that the existence of a pdf 
and the representations (2) and (3) hold under linear growth condition on $b$. By using these representations, we show that Gaussian two-sided bound and Hölder continuity hold under sub-linear growth condition on $b$.

As an application of the representation of a pdf, we consider the rate of convergence for a pdf of the Euler-Maruyama scheme, which is a standard discrete approximation for a solution of SDEs (see, [30, 44]). It is worth noting that Maruyama's proof of Girsanov's theorem in [43] is based on this approximation. For the Euler-Maruyama scheme $X^{(x, n)}$, many authors studies the strong error $\mathbb{E}\left[\sup _{0 \leq t<T}\left|X_{t}^{x}-X_{t}^{(x, n)}\right|^{p}\right]^{1 / p}$, for some $p \geq 1$ and the weak error $\left|\mathbb{E}\left[f\left(X_{T}^{x}\right)\right]-\mathbb{E}\left[f\left(X_{T}^{(x, n)}\right)\right]\right|$ for some measurable function $f: \mathbb{R}^{d} \rightarrow \mathbb{R}$, (see, e.g. [5, 8, 30, 33, 40, 44, 46, 47, 49, 50, 64, 66, see also [1, 4, 12, 23, 26, 31, 34 as an unbiased simulation scheme based on the Euler-Maruyama scheme with random grid and parametrix expansions/Malliavin weight). On the other hand, recently, the convergence rate for a pdf of the Euler-Maruyama scheme studied by using the parametrix method and Malliavin calculus (see, e.g. [19, 36, 20, 50]). In this paper, we provide the rate of convergence by using the representation of (2) for pdfs of Brownian motion with path-dependent drift and its Euler-Maruyama approximation.

Recently, if the coefficients of SDE grow super-linearly, then the standard Euler-Maruyama approximation does not converge to a solution of the equation (see, Theorem 2.1 in [24]). In order to approximate a solution of Markov type SDE with super-linear growth coefficients, several tamed Euler-Maruyama approximations are proposed (see e.g., [25, 58, 59]). In this paper, inspired by [25, 58, 59], we use arguments of Fournier and Printems [14] with "one-step" tamed EulerMaruyama approximation in order to prove absolute continuity of the law of $X_{t}^{x}$ with respect to the Lebesgue measure under super-linear growth, Khasminskii and one-sided Lipschitz condition on the coefficients. It is worth noting that Romito [57] studied the existence and Besov regularity of the probability density function of a solution of SDEs with locally bounded drift, and locally Hölder continuous, elliptic diffusion coefficient, by using localization argument and one-step EulerMaruyama scheme. Therefore, the result proved in section 4 is included in the result of Romito (see, Theorem 4.1 in [57]). On the other hand, in our paper, we use directly one-step tamed Euler scheme, so the approach of proof is different.

This paper is structured as follows. In section 2, we prove the existence and weak uniqueness of a solution of SDE (1) with linear growth and path-dependent drift coefficient. In section 3 , we study a pdf of a solution of SDEs (1). We first provide two representations for a pdf in subsection 3.1. and prove a Gaussian two-sided bound in subsection 3.2. In subsection 3.3. we provide third representation for a pdf of a solution of SDEs with path-dependent and bounded drift, and as an application of this representation, in subsection [3.4, we prove a sharp bounds for a pdf of Brownian motion with drift which is inspired by [54, 55. (see also, 6]). In subsection [3.5. we consider a comparison property of pdfs, and in subsection 3.6. as an application of a comparison property for pdfs of a solution of SDE and its Euler-Maruyama scheme, we provide its rate of convergence. In subsection 3.7, we consider the parametrix method for a pdf of a solution of Markovian SDEs with unbounded drift, and in subsection 3.8 as an application of the parametrix method, we provide an unbiased simulation scheme introduced by Bally and Kohatsu-Higa [4]. In subsection 3.9, we study Hölder continuity of a pdf by using the explicit representation. In section 4. we prove the existence of a pdf of a solution of one-dimensional SDEs under super-linear growth conditions on the coefficients. In a short Appendix, we provide an explicit calculation for beta type integrals. 


\section{Notations}

We give some basic notations and definitions used throughout this paper. We consider the elements of $\mathbb{R}^{d}$ are column vectors and for $x \in \mathbb{R}^{d}$, we denote $x=\left(x^{1}, \ldots, x^{d}\right)^{\top}$. Let $T>0$ be fixed. We denote by $C\left([0, \infty) ; \mathbb{R}^{d}\right)$ the space of continuous functions $w:[0, \infty) \rightarrow \mathbb{R}^{d}$ with metric $\rho$ defined by $\rho\left(w, w^{\prime}\right)=\sum_{k=1}^{\infty} 2^{-k}\left(\max _{0 \leq t \leq k}\left|w_{t}-w_{t}^{\prime}\right| \wedge 1\right)$. Let us denote by $\mathcal{B}\left(C\left([0, \infty) ; \mathbb{R}^{d}\right)\right)$ the topological $\sigma$-field on $C\left([0, \infty) ; \mathbb{R}^{d}\right)$, and $\mathcal{B}_{t}\left(C\left([0, \infty) ; \mathbb{R}^{d}\right)\right)$ the sub- $\sigma$-field defined by $\left\{\rho_{t}^{-1}(A) \mid A \in\right.$ $\left.\mathcal{B}\left(C\left([0, \infty) ; \mathbb{R}^{d}\right)\right)\right\}$, where $\rho_{t}(w)(s):=w(t \wedge s)$, (see, Chapter IV in [27]). For $w \in C\left([0, \infty) ; \mathbb{R}^{d}\right)$, define $w_{t}^{*}:=\sup _{0 \leq s \leq t}\left|w_{s}\right|$. Let $C_{b}^{\infty}\left(\mathbb{R}^{d} ; \mathbb{R}^{q}\right)$ be the space of $\mathbb{R}^{q}$-valued functions such that all the derivatives are bounded. For an invertible $d \times d$-matrix $A=\left(A_{i, j}\right)_{1 \leq i, j \leq d}$, we denote $|A|^{2}:=$ $\sum_{i, j=1}^{d} A_{i, j}^{2}$ and $g_{A}(x, y)=\frac{\exp \left(-\frac{1}{2}\left\langle A^{-1}(y-x), y-x\right\rangle\right)}{(2 \pi)^{d / 2} \sqrt{\operatorname{det} A}}$, and $g_{c}(x, y)=g_{c I}(x, y)$, for $c \in \mathbb{R} \backslash\{0\}$ where the matrix $I$ is the identity matrix. We denote the sign function by $\operatorname{sgn}(x):=-\mathbf{1}_{(-\infty, 0]}(x)+\mathbf{1}_{(0, \infty)}(x)$ for $x \in \mathbb{R}$, and the gamma function by $\Gamma(x):=\int_{0}^{\infty} t^{x-1} e^{-t} \mathrm{~d} t$ for $x \in(0, \infty)$. We will use Hermite polynomials associated with the Gaussian density of order 1 and 2 denoted by $H^{i}$ and $H^{i, j}$, that is, for an invertible matrix $A, H_{A}^{i}(y):=-\left(A^{-1} y\right)^{i}$ and $H_{A}^{i, j}(y):=\left(A^{-1} y\right)^{i}\left(A^{-1} y\right)^{j}-\left(A^{-1}\right)_{i, j}$.

\section{SDEs with unbounded and path-dependent drift}

In this section, we will show that weak existence and uniqueness in law for a solution of SDE (1) on $[0, T]$.

It is known that if $\sigma$ is identity matrix $I_{d}$ and $b:[0, T] \times C\left([0, T] ; \mathbb{R}^{d}\right) \rightarrow \mathbb{R}^{d}$ is a progressively measurable functional on $C\left([0, T] ; \mathbb{R}^{d}\right)$ satisfying the linear growth condition $|b(t, w)| \leq K\left(1+w_{t}^{*}\right)$, for all $(t, w) \in[0, T] \times C\left([0, T] ; \mathbb{R}^{d}\right)$, then there exists a unique weak solution of SDE (1) (see, Proposition 5.3.6 and Remark 5.3.8 in [28]). The idea of proof is based on Maruyama-Girsanov transform with "local" Novikov condition (see, Corollary 3.5.14 and 3.5.16 in [28]). In order to extend this result to non-constant diffusion matrix, we use the Gaussian upper bound for a pdf of a solution of SDE without drift coefficient (see, (5) below).

We need the following assumptions on the coefficients $b$ and $\sigma$.

Assumption 2.1. We suppose that the coefficients $b=\left(b^{(1)}, \ldots, b^{(d)}\right)^{\top}:[0, \infty) \times C\left([0, \infty) ; \mathbb{R}^{d}\right) \rightarrow$ $\mathbb{R}^{d}$ and $\sigma=\left(\sigma_{i, j}\right)_{1 \leq i, j \leq d}:[0, \infty) \times \mathbb{R}^{d} \rightarrow \mathbb{R}^{d \times d}$ satisfy the following conditions:

(i) The drift coefficient $b$ is $\mathcal{B}([0, \infty)) \otimes \mathcal{B}\left(C\left([0, \infty) ; \mathbb{R}^{d}\right)\right) / \mathcal{B}\left(\mathbb{R}^{d}\right)$-measurable and for each fixed $t>0$, the map $C\left([0, \infty) ; \mathbb{R}^{d}\right) \ni w \mapsto b(t, w) \in \mathbb{R}^{d}$ is $\mathcal{B}_{t}\left(C\left([0, \infty) ; \mathbb{R}^{d}\right)\right) / \mathcal{B}\left(\mathbb{R}^{d}\right)$-measurable (see, Chapter IV, Definition 1.1 in [27]), and is of linear growth, that is, for each $T>0$, there exists $K(b, T)>0$ such that for any $(t, w) \in[0, T] \times C\left([0, T] ; \mathbb{R}^{d}\right)$,

$$
|b(t, w)| \leq K(b, T)\left(1+w_{t}^{*}\right)
$$

(ii) $a:=\sigma \sigma^{\top}$ is $\alpha$-Hölder continuous in space and $\alpha / 2$-Hölder continuous in time with $\alpha \in(0,1]$, that is,

$$
\|a\|_{\alpha}:=\sup _{t \in[0, \infty), x \neq y} \frac{|a(t, x)-a(t, y)|}{|x-y|^{\alpha}}+\sup _{x \in \mathbb{R}^{d}, t \neq s} \frac{|a(t, x)-a(s, x)|}{|t-s|^{\alpha / 2}}<\infty
$$


(iii) The diffusion coefficient $\sigma$ is bounded and uniformly elliptic, that is, there exist $\underline{a}, \bar{a}>0$ such that for any $(t, x, \xi) \in[0, \infty) \times \mathbb{R}^{d} \times \mathbb{R}^{d}$,

$$
\underline{a}|\xi|^{2} \leq\langle a(t, x) \xi, \xi\rangle \leq \bar{a}|\xi|^{2} .
$$

Example 2.1. Suppose that $\nu: \mathbb{S}:=[0, \infty) \times \mathbb{R}^{d} \times[0, \infty) \times\left(\mathbb{R}^{d}\right)^{\mathbb{N}} \times \mathbb{R}^{\ell} \rightarrow \mathbb{R}^{d}$ is measurable and satisfies that there exists $K>0$ and $\theta:=\left\{\theta_{i}\right\}_{i \in \mathbb{N}} \in[0, \infty)^{\mathbb{N}}$ such that $\|\theta\|_{\ell_{1}}:=\sum_{i \in \mathbb{N}} \theta_{i}<\infty$ and for any $\chi=\left(t, w, z,\left\{u_{i}\right\}_{i \in \mathbb{N}}, v\right) \in \mathbb{S}$,

$$
|\nu(\chi)| \leq K\left(1+\|\chi\|_{\theta}\right)
$$

where $\|\chi\|_{\theta}^{2}:=t^{2}+|w|^{2}+|z|^{2}+\sum_{i \in \mathbb{N}} \theta_{i}\left|u_{i}\right|^{2}+|v|^{2}$. We define $A_{t}: C\left([0, \infty) ; \mathbb{R}^{d}\right) \rightarrow \mathbb{S}$ by

$$
A_{t}(w)=\left(t, w_{t}, \max _{0 \leq s \leq t} \zeta\left(s, w_{s}\right),\left\{w_{\tau_{i}(t)}\right\}_{i \in \mathbb{N}}, \int_{0}^{t} c\left(s, w_{s}\right) \mathrm{d} s,\right) \in \mathbb{S}, w \in C\left([0, \infty) ; \mathbb{R}^{d}\right),
$$

where $\tau_{i}(t):=\left(t-\tau_{i}\right) \mathbf{1}_{\left(\tau_{i}, \infty\right)}(t), \tau_{i}>0, i \in \mathbb{N}, \zeta:[0, \infty) \times \mathbb{R}^{d} \rightarrow[0, \infty)$ and $c:[0, \infty) \times \mathbb{R}^{d} \rightarrow \mathbb{R}^{\ell}$ are measurable and of linear growth. Then since $\zeta$ and $c$ are of linear growth, there exists $C>0$ such that for any $w \in C\left([0, \infty) ; \mathbb{R}^{d}\right),\left\|A_{t}(\omega)\right\|_{\theta} \leq C\left(1+\left(1+\|\theta\|_{\ell_{1}}\right) w_{t}^{*}\right)$, thus $b=\nu \circ$ A. satisfies Assumption 2.1 (i).

Recall that we fixed $T>0$ arbitrarily. Let us consider the following Markovian SDE without drift coefficient: for given $s \in[0, T)$,

$$
Y_{t}^{s, x}=x+\int_{s}^{t} \sigma\left(r, Y_{r}^{s, x}\right) \mathrm{d} W_{r}, t \in[s, T] .
$$

If the diffusion coefficient $\sigma$ satisfies the Assumption 2.1 (ii) and (iii), then a weak existence and uniqueness in law holds (see, e.g. Theorem 4.1 and 5.6 in [62]). Moreover, from Theorem 5.4 in [16], $Y_{t}^{s, x}$ admits the pdf (fundamental solution) $q(s, x ; t, \cdot)$ for any $t \in(s, T]$ and $q(s, x ; t, y)$ is the solution of the Kolmogorov backward equation:

$$
\left(\partial_{s}+L_{s}\right) q(s, x ; t, y)=0, \lim _{s \uparrow t} \int_{\mathbb{R}^{d}} f(y) q(s, x ; t, y) \mathrm{d} y=f(x), f \in C_{b}^{\infty}\left(\mathbb{R}^{d} ; \mathbb{R}\right),
$$

where $L_{s}$ is a differential operator defined by

$$
L_{s} f(x):=\frac{1}{2} \sum_{i, j=1}^{d} a_{i, j}(s, x) \frac{\partial^{2} f}{\partial x_{i} \partial x_{j}}(x),
$$

(see page 149 in [16]). Moreover, for any $f \in C_{b}^{\infty}\left(\mathbb{R}^{d} ; \mathbb{R}\right)$, the function $u(s, x ; t):=\mathbb{E}\left[f\left(Y_{t}^{s, x}\right)\right]$ is a solution to the following partial differential equation:

$$
\begin{aligned}
\left(\partial_{s}+L_{s}\right) u(s, x ; t) & =0, \quad(s, x) \in[0, t) \times \mathbb{R}^{d}, \\
u(t, x ; t) & =f(x), x \in \mathbb{R}^{d},
\end{aligned}
$$

(see, Theorem 5.3 in [16]).

The following lemma shows that $q(s, x ; t, \cdot)$ and its derivative with respect to $x$ satisfy the Gaussian bounded. 
Lemma 2.2. Suppose that Assumption[2.1-(ii) and (iii) holds. Then for all $t \in(s, T], Y_{t}^{s, x}$ admits the pdf $q(s, x ; t, \cdot)$ with respect to Lebesgue measure, and there exist $\widehat{C}_{ \pm}>0$ and $\widehat{c}_{ \pm}>0$ such that for any $(t, x, y) \in(s, T] \times \mathbb{R}^{d} \times \mathbb{R}^{d}$,

$$
\begin{aligned}
\widehat{C}_{-} g_{\widehat{c}_{-}(t-s)}(x, y) \leq q(s, x ; t, y) & \leq \widehat{C}_{+} g_{\widehat{c}_{+}(t-s)}(x, y), \\
\left|\partial_{x_{i}} q(s, x ; t, y)\right| & \leq \frac{\widehat{C}_{+}}{(t-s)^{1 / 2}} g_{\widehat{c}_{+}(t-s)}(x, y) .
\end{aligned}
$$

The upper bound (8) and (9) are shown in [15, Theorem 9.4.2. For the lower bound (8), we refer section 4.2 in [39, and 60] for time independent case, see also Chapter 7, section 6 in [9].

By using the Gaussian upper bound (8), we prove that the existence and uniqueness in law holds for SDE (1) under linear growth condition on $b$.

Theorem 2.3. Suppose that Assumption 2.1 holds. Then SDE (1) has a weak solution and uniqueness in law holds on $[0, T]$. In particular, for any measurable functional $f: C\left([0, T] ; \mathbb{R}^{d}\right) \rightarrow \mathbb{R}$ such that the expectation $\mathbb{E}\left[f\left(Y^{0, x}\right) Z_{T}\left(1, Y^{0, x}\right)\right]$ exists, it holds that

$$
\mathbb{E}\left[f\left(X^{x}\right)\right]=\mathbb{E}\left[f\left(Y^{0, x}\right) Z_{T}\left(1, Y^{0, x}\right)\right],
$$

where for $q \in \mathbb{R}, Z\left(q, Y^{0, x}\right)=\left(Z_{t}\left(q, Y^{0, x}\right)\right)_{t \in[0, T]}$ is a martingale defined by

$$
\begin{aligned}
Z_{t}\left(q, Y^{0, x}\right) & :=\exp \left(\sum_{j=1}^{d} \int_{0}^{t} q \mu^{j}\left(s, Y^{0, x}\right) \mathrm{d} W_{s}^{j}-\frac{1}{2} \int_{0}^{t}\left|q \mu\left(s, Y^{0, x}\right)\right|^{2} \mathrm{~d} s\right), \\
\mu(t, w) & :=\sigma\left(t, w_{t}\right)^{-1} b(t, w),(t, w) \in[0, T] \times C\left([0, T] ; \mathbb{R}^{d}\right) .
\end{aligned}
$$

Before proving Theorem 2.3 , we prove the following lemma which shows that the random variable $X_{T}^{x, *}$ is $L^{p}$-integrable for all $p>0$.

Lemma 2.4. Assume that $F:[0, \infty) \times C\left([0, \infty) ; \mathbb{R}^{d}\right) \rightarrow \mathbb{R}^{d}$ is $\mathcal{B}([0, \infty)) \otimes \mathcal{B}\left(C\left([0, \infty) ; \mathbb{R}^{d}\right)\right) / \mathcal{B}\left(\mathbb{R}^{d}\right)$ measurable and for each fixed $t>0$, the map $C\left([0, \infty) ; \mathbb{R}^{d}\right) \ni w \mapsto F(t, w) \in \mathbb{R}^{d}$ is $\mathcal{B}_{t}\left(C\left([0, \infty) ; \mathbb{R}^{d}\right)\right) / \mathcal{B}\left(\mathbb{R}^{d}\right)$ measurable, and is of linear growth, that is, for each $T>0$, there exists $K(F, T)>0$ such that for any $(t, w) \in[0, T] \times C\left([0, T] ; \mathbb{R}^{d}\right)$,

$$
|F(t, w)| \leq K(F, T)\left(1+w_{t}^{*}\right) .
$$

Suppose that Assumption 2.1 holds and let $X^{x}$ be a solution of SDE (11). Then for any $p>0$, there exists $C_{b, \sigma}(p, F, T)>0$ such that

$$
\mathbb{E}\left[\sup _{t \in[0, T]}\left|F\left(t, X^{x}\right)\right|^{p}\right]^{1 / p} \leq C_{b, \sigma}(p, F, T)(1+|x|) .
$$

Proof. It is suffices to show the statement for $p \geq 2$. Since $b$ is of linear growth and $\sigma$ is bounded, applying Jensen's inequality and Burkholder-Davis-Gundy's inequality, there exist $\widetilde{C}_{1}, \widetilde{C}_{2}>0$ such that for all $t \in[0, T]$,

$$
\mathbb{E}\left[\left|X_{t}^{x, *}\right|^{p}\right] \leq \widetilde{C}_{1}\left(1+|x|^{p}\right)+\widetilde{C}_{2} \int_{0}^{t} \mathbb{E}\left[\left|X_{s}^{x, *}\right|^{p}\right] \mathrm{d} s .
$$

Hence Gronwall's inequality implies the statement. 
Proof of Theorem 2.3. The proof is based on Corollary 3.5.16, Proposition 5.3.6, Remark 5.3.8 and Proposition 3.10 in [28.

We first prove that $Z\left(q, Y^{0, x}\right)$ is a martingale for all $q \in \mathbb{R}$. Since usual Novikov condition may be fail in this setting, we apply a local Novikov condition as follows. From Corollary 3.5.14 in [28, it suffices to prove that for any fixed $T>0$, there exist $n(T) \in \mathbb{N}$ and a sequence $\left\{t_{0}, \ldots, t_{n(T)}\right\}$ such that $0=t_{0}<t_{1}<\cdots<t_{n(T)}=T$ and

$$
\mathbb{E}\left[\exp \left(\frac{1}{2} \int_{t_{n-1}}^{t_{n}}\left|q \mu\left(s, Y^{0, x}\right)\right|^{2} \mathrm{~d} s\right)\right]<\infty, \text { for all } n=1, \ldots, n(T) .
$$

Let $M_{t}^{x}:=Y_{t}^{0, x}-x$. Then $M^{x}=\left(M_{t}^{x}\right)_{t \in[0, T]}$ is a martingale since $\sigma$ is bounded. By Assumption 2.1 (i) and (iii), we have

$$
\int_{t_{n-1}}^{t_{n}}\left|q \mu\left(s, Y^{0, x}\right)\right|^{2} \mathrm{~d} s \leq\left(t_{n}-t_{n-1}\right) \underline{a}|q K(b, T)|^{2}\left(1+|x|+M_{T}^{x, *}\right)^{2} .
$$

Note that $U_{t}^{x}:=\exp \left(\frac{1}{4}\left(t_{n}-t_{n-1}\right) \underline{a}|q K(b, T)|^{2}\left(1+|x|+\left|M_{t}^{x}\right|\right)^{2}\right)$ is a sub-martingale, so by using Doob's inequality (see, Theorem 1.3.8 (iv) in [28]), we have

$$
\begin{aligned}
\mathbb{E}\left[\exp \left(\frac{1}{2} \int_{t_{n-1}}^{t_{n}}\left|q \mu\left(r, Y^{0, x}\right)\right|^{2} \mathrm{~d} r\right)\right] & \leq \mathbb{E}\left[\exp \left(\frac{1}{2}\left(t_{n}-t_{n-1}\right) \underline{a}|q K(b, T)|^{2}\left(1+|x|+M_{T}^{x, *}\right)^{2}\right)\right] \\
& =\mathbb{E}\left[\left|U_{T}^{x, *}\right|^{2}\right] \leq 4 \mathbb{E}\left[\left|U_{T}^{x}\right|^{2}\right] .
\end{aligned}
$$

Using the Gaussian upper bound (8), we have

$$
\begin{aligned}
\mathbb{E}\left[\left|U_{T}^{x}\right|^{2}\right] & =\int_{\mathbb{R}^{d}} \exp \left(\frac{1}{2}\left(t_{n}-t_{n-1}\right) \underline{a}|q K(b, T)|^{2}(1+|x|+|y-x|)^{2}\right) q(0, x ; T, y) \mathrm{d} y \\
& \leq \widehat{C}_{+} \int_{\mathbb{R}^{d}} \exp \left(\frac{1}{2}\left(t_{n}-t_{n-1}\right)\left\{2 \underline{a}|q K(b, T)|^{2}\right\}\left\{(1+|x|)^{2}+|y-x|^{2}\right\}\right) g_{\widehat{c}_{+} T}(x, y) \mathrm{d} y .
\end{aligned}
$$

We choose $n(T) \in \mathbb{N}$ and the sequence $\left\{t_{0}, \ldots, t_{n(T)}\right\}$ satisfying

$$
t_{n}-t_{n-1} \leq \frac{1}{2 \underline{a}|q K(b, T)|^{2} \widehat{c}_{+} T},
$$

which provides $\mathbb{E}\left[\left|U_{T}^{x}\right|^{2}\right]<\infty$. This concludes that $Z\left(q, Y^{0, x}\right)$ is a martingale.

We define the new measure $\mathbb{Q}$ on the measurable space $\left(\Omega, \mathcal{F}_{T}\right)$ as

$$
\frac{\mathrm{d} \mathbb{Q}}{\mathrm{d} \mathbb{P}}=Z_{T}\left(1, Y^{0, x}\right) .
$$

Since $Z\left(1, Y^{0, x}\right)$ is a martingale, the measure $\mathbb{Q}$ is a probability measure on $\left(\Omega, \mathcal{F}_{T}\right)$. Moreover, from Maruyama-Girsanov theorem, $B=\left(B_{t}=\left(B_{t}^{1}, \ldots, B_{t}^{d}\right)^{\top}\right)_{0 \leq t \leq T}$, which is defined by for each $j=1, \ldots, d$,

$$
B_{t}^{j}:=W_{t}^{j}-\left\langle W^{j}, \sum_{\ell=1}^{d} \int_{0}^{\cdot} \mu^{\ell}\left(s, Y^{0, x}\right) \mathrm{d} W_{s}^{\ell}\right\rangle_{t}=W_{t}^{j}-\int_{0}^{t} \mu^{j}\left(s, Y^{x}\right) \mathrm{d} s,
$$


is a $d$-dimensional standard Brownian motion on the probability space $\left(\Omega, \mathcal{F}_{T}, \mathbb{Q}\right)$. Hence we have

$$
\mathrm{d} Y_{t}^{0, x}=\sigma\left(t, Y_{t}^{0, x}\right) \mathrm{d} W_{t}=b\left(t, Y^{0, x}\right) \mathrm{d} t+\sigma\left(t, Y_{t}^{0, x}\right) \mathrm{d} B_{t},
$$

thus, $Y^{x}$ is a solution of SDE (11) with $Y_{0}^{0, x}=x$ under the probability measure $\mathbb{Q}$.

Next, we prove the uniqueness in law. The proof is based on Proposition 5.3.10 in [28. Let $\left(X^{i}, W^{i}\right),\left(\Omega^{i}, \mathcal{F}^{i}, \mathbb{P}^{i}\right),\left\{\mathcal{F}_{t}^{i}\right\}_{t \geq 0}, i=1,2$ be two solution of SDE (11). For each $k \geq 1$, let

$$
\tau_{k}^{i}:=T \wedge \inf \left\{t \in[0, T] ; \int_{0}^{t}\left|\mu\left(s, X^{i}\right)\right|^{2} \mathrm{~d} s=k\right\} .
$$

From Lemma 2.4 $\tau_{k}^{i} \rightarrow T$ as $k \rightarrow \infty$, almost surely. Then, for each $k \in \mathbb{N}$ and $i=1,2$ $\left(Z_{t \wedge \tau_{k}^{i}}\left(-1, X^{i}\right)\right)_{t \in[0, T]}$ is a martingale on $\left(\Omega^{i}, \mathcal{F}_{T}^{i}, \mathbb{P}^{i}\right)$. For each $i=1,2$, we define new a measure on $\left(\Omega^{i}, \mathcal{F}_{T}^{i}\right)$ as

$$
\frac{\mathrm{d} \mathbb{Q}_{k}^{i}}{\mathrm{~d} \mathbb{P}^{i}}=Z_{T \wedge \tau_{k}^{i}}\left(-1, X^{i}\right)
$$

Then from Maruyama-Girsanov theorem, for $i=1,2,\left(B_{t \wedge \tau_{k}^{i}}^{i}=\left(B_{t \wedge \tau_{k}^{i}}^{i, 1}, \ldots, B_{t \wedge \tau_{k}^{i}}^{i, d}\right)^{\top}\right)_{0 \leq t \leq T}$, which is defined by for each $j=1, \ldots, d, B_{t}^{i, j}:=W_{t}^{i, j}-\int_{0}^{t} \mu^{j}\left(s, X^{i}\right) \mathrm{d} s$, are $d$-dimensional standard Brownian motion on the probability space $\left(\Omega^{i}, \mathcal{F}_{T}^{i}, \mathbb{Q}_{k}^{i}\right)$, and then, for each $k \geq 1$ and $i=1,2$, the process $\left(X_{t \wedge \tau_{k}^{i}}^{i}, B_{t \wedge \tau_{k}^{i}}^{i}\right)_{0 \leq t \leq T}$ is a solution of (5) under $\mathbb{Q}_{k}^{i}$. By the same way of Proposition 3.10 in 28], the uniqueness in law for SDE (5) implies the uniqueness in law for SDE (10).

Finally, we prove (10). By the uniqueness in law of $X^{x}$, for any measurable functional $f$ : $C\left([0, T] ; \mathbb{R}^{d}\right) \rightarrow \mathbb{R}$ such that the expectation $\mathbb{E}\left[f\left(Y^{x}\right) Z_{T}\left(1, Y^{x}\right)\right]$ exists, we have

$$
\mathbb{E}\left[f\left(X^{x}\right)\right]=\mathbb{E}_{\mathbb{Q}}\left[f\left(Y^{0, x}\right)\right]=\mathbb{E}\left[f\left(Y^{0, x}\right) \frac{\mathrm{d} \mathbb{Q}}{\mathrm{dP}}\right]=\mathbb{E}\left[f\left(Y^{0, x}\right) Z_{T}\left(1, Y^{0, x}\right)\right],
$$

which concludes the proof.

\section{PDF of a solution of SDEs with unbounded and path- dependent drift}

In this section, we show that the existence, representation, Gaussian two-sided bound and Hölder continuity for a pdf of a solution of SDEs with unbounded and path-dependent drift coefficient.

\subsection{Existence and representations}

We obtain the existence and representations for a pdf of a solution of SDE (1) under linear growth condition on $b$. 
Theorem 3.1. Suppose Assumption [2.1 holds. Then for any $(t, x) \in(0, T] \times \mathbb{R}^{d}, X_{t}^{x}$ admits a $p d f$, denoted by $p_{t}(x, \cdot)$, with respect to Lebesgue measure and it has the following representations

$$
\begin{aligned}
p_{t}(x, y) & =q(0, x ; t, y)+\int_{0}^{t} \mathbb{E}\left[\left\langle\nabla_{x} q\left(s, X_{s}^{x} ; t, y\right), b\left(s, X^{x}\right)\right\rangle\right] \mathrm{d} s, \text { a.e., } y \in \mathbb{R}^{d}, \\
& =q(0, x ; t, y) \mathbb{E}\left[Z_{t}\left(1, Y^{0, x}\right) \mid Y_{t}^{0, x}=y\right], \text { a.e., } y \in \mathbb{R}^{d},
\end{aligned}
$$

where $\mathbb{E}\left[\cdot \mid Y_{t}^{0, x}=y\right]$ is the expectation of a regular conditional probability given $Y_{t}^{0, x}=y$ for $y \in \mathbb{R}^{d}$.

Proof. We first show the second representation (12). From Theorem 2.3, $Z\left(1, Y^{0, x}\right)$ is a martingale, thus for any $f \in C_{b}^{\infty}\left(\mathbb{R}^{d} ; \mathbb{R}\right)$, it holds that

$$
\mathbb{E}\left[f\left(X_{t}^{x}\right)\right]=\mathbb{E}\left[f\left(Y_{t}^{0, x}\right) Z_{T}\left(1, Y^{0, x}\right)\right]=\mathbb{E}\left[f\left(Y_{t}^{0, x}\right) \mathbb{E}\left[Z_{T}\left(1, Y^{0, x}\right) \mid \mathcal{F}_{t}\right]\right]=\mathbb{E}\left[f\left(Y_{t}^{0, x}\right) Z_{t}\left(1, Y^{0, x}\right)\right] .
$$

On the other hand, from Theorem 1.3.3 in [27, there exists a regular conditional probability given $Y_{t}^{0, x}=y$ for $y \in \mathbb{R}^{d}$, denoted by $\mathbb{P}\left(\cdot \mid Y_{t}^{0, x}=y\right)$, such that

$$
\mathbb{E}\left[f\left(Y_{t}^{0, x}\right) Z_{t}\left(1, Y^{0, x}\right)\right]=\int_{\mathbb{R}^{d}} f(y) \mathbb{E}\left[Z_{t}\left(1, Y^{0, x}\right) \mid Y_{t}^{0, x}=y\right] \mathbb{P}\left(Y_{t}^{0, x} \in \mathrm{d} y\right),
$$

where $\mathbb{E}\left[\cdot \mid Y_{t}^{0, x}=y\right]$ is the expectation with respect to $\mathbb{P}\left(\cdot \mid Y_{t}^{0, x}=y\right)$. Therefore, it holds that for each fixed $(t, x) \in(0, T] \times \mathbb{R}^{d}$,

$$
p_{t}(x, y)=q(0, x ; t, y) \mathbb{E}\left[Z_{t}\left(1, Y^{0, x}\right) \mid Y_{t}^{0, x}=y\right] \in[0, \infty) \text {, a.e., } y \in \mathbb{R}^{d},
$$

which is the second representation (12).

Now we show the first representation (11). It suffices to prove that for any $f \in C_{b}^{\infty}\left(\mathbb{R}^{d} ; \mathbb{R}\right)$,

$$
\mathbb{E}\left[f\left(X_{t}^{x}\right)\right]=\int_{\mathbb{R}^{d}} f(y)\left\{q(0, x ; t, y)+\int_{0}^{t} \mathbb{E}\left[\left\langle\nabla_{x} q\left(s, X_{s}^{x} ; t, y\right), b(s, X)\right\rangle\right] \mathrm{d} s\right\} \mathrm{d} y .
$$

By the definition of $u(s, x ; t)$, we have

$$
\mathbb{E}\left[f\left(Y_{t}^{0, x}\right)\right]=u(0, x ; t), \quad \mathbb{E}\left[f\left(X_{t}^{x}\right)\right]=\mathbb{E}\left[u\left(t, X_{t}^{x} ; t\right)\right] .
$$

By using Itô's formula, it holds that for any $\varepsilon \in(0, t)$,

$$
\begin{aligned}
u\left(t-\varepsilon, X_{t-\varepsilon}^{x} ; t\right)= & u(0, x ; t)+\int_{0}^{t-\varepsilon}\left(\partial_{s}+L_{s}\right) u\left(s, X_{s}^{x} ; t\right) \mathrm{d} s+\int_{0}^{t-\varepsilon}\left\langle\nabla_{x} u\left(s, X_{s}^{x} ; t\right), b\left(s, X^{x}\right)\right\rangle \mathrm{d} s \\
& +\sum_{i, j=1}^{d} \int_{0}^{t-\varepsilon} \sigma_{i, j}\left(s, X_{s}^{x}\right) \partial_{x_{i}} u\left(s, X_{s}^{x} ; t\right) \mathrm{d} W_{s}^{j} .
\end{aligned}
$$

Since $u(s, x ; t)$ is a solution to the heat equation (7), it holds that

$$
u\left(t-\varepsilon, X_{t-\varepsilon}^{x} ; t\right)=u(0, x ; t)+\int_{0}^{t-\varepsilon}\left\langle\nabla_{x} u\left(s, X_{s}^{x} ; t\right), b(s, X)\right\rangle \mathrm{d} s
$$




$$
+\sum_{i, j=1}^{d} \int_{0}^{t-\varepsilon} \sigma_{i, j}\left(s, X_{s}^{x}\right) \partial_{x_{i}} u\left(s, X_{s}^{x} ; t\right) \mathrm{d} W_{s}^{j} .
$$

Since for $i=1, \ldots, d$, by using (9) and dominated convergence theorem,

$$
\partial_{x_{i}} u(s, x ; t)=\partial_{x_{i}} \mathbb{E}\left[f\left(Y_{t}^{s, x}\right)\right]=\int_{\mathbb{R}^{d}} f(y) \frac{\partial}{\partial x_{i}} q(s, x ; t, y) \mathrm{d} y,
$$

and it holds that for any $s \in[0, t)$ and $x \in \mathbb{R}^{d}$,

$$
\left|\partial_{x_{i}} u(s, x ; t)\right| \leq \frac{C\|f\|_{\infty}}{(t-s)^{1 / 2}},
$$

for some $C>0$. Therefore, the martingale property implies that the expectation of $\int_{0}^{t-\varepsilon} \sigma_{i, j}\left(s, X_{s}^{x}\right) \partial_{i} u\left(s, X_{s}^{x} ; t\right) \mathrm{d} W_{s}^{j}$ equals to zero, and by using Schwarz's inequality and Lemma 2.4 with $F=b$ and $p=1$,

$$
\mathbb{E}\left[\int_{0}^{t}\left|\left\langle\nabla_{x} u\left(s, X_{s}^{x} ; t\right), b\left(s, X^{x}\right)\right\rangle\right| \mathrm{d} s\right] \leq \sqrt{d} C\|f\|_{\infty} \mathbb{E}\left[\int_{0}^{t} \frac{\left|b\left(s, X^{x}\right)\right|}{(t-s)^{1 / 2}} \mathrm{~d} s\right]<\infty .
$$

Hence, by taking the expectation and Fubini's theorem, we have from (14) and (16)

$$
\mathbb{E}\left[u\left(t-\varepsilon, X_{t-\varepsilon}^{x} ; t\right)\right]=\mathbb{E}\left[f\left(Y_{t}^{0, x}\right)\right]+\int_{0}^{t-\varepsilon} \mathbb{E}\left[\left\langle\nabla_{x} u\left(s, X_{s}^{x} ; t\right), b\left(s, X^{x}\right)\right\rangle\right] \mathrm{d} s .
$$

Since $\sup _{(s, x) \in[0, t] \times \mathbb{R}^{d}}|u(s, x ; t)| \leq\|f\|_{\infty}$, by using the dominated convergence theorem and (16), (17),

$$
\begin{aligned}
\mathbb{E}\left[f\left(X_{t}^{x}\right)\right] & =\mathbb{E}\left[\lim _{\varepsilon \rightarrow 0+} u\left(t-\varepsilon, X_{t-\varepsilon}^{x} ; t\right)\right]=\lim _{\varepsilon \rightarrow 0+} \mathbb{E}\left[u\left(t-\varepsilon, X_{t-\varepsilon}^{x} ; t\right)\right] \\
& =\mathbb{E}\left[f\left(Y_{t}^{0, x}\right)\right]+\int_{0}^{t} \mathbb{E}\left[\left\langle\nabla_{x} u\left(s, X_{s}^{x} ; t\right), b\left(s, X^{x}\right)\right\rangle\right] \mathrm{d} s .
\end{aligned}
$$

Finally, from Lemma 2.4 with $F=b$ and $p=1$,

$$
\begin{aligned}
& \sum_{i=1}^{d} \int_{0}^{t} \int_{\mathbb{R}^{d}} \mathbb{E}\left[|f(y)|\left|\frac{\partial}{\partial x_{i}} q\left(s, X_{s}^{x} ; t, y\right)\right|\left|b^{i}\left(s, X^{x}\right)\right|\right] \mathrm{d} y \mathrm{~d} s \\
& \leq \sqrt{d} \widehat{C}_{+}\|f\|_{\infty} \int_{0}^{t} \int_{\mathbb{R}^{d}} \frac{1}{(t-s)^{1 / 2}} \mathbb{E}\left[g_{\widehat{c}_{+}(t-s)}\left(X_{s}^{x}, y\right)\left|b\left(s, X^{x}\right)\right|\right] \mathrm{d} y \mathrm{~d} s \\
& =\sqrt{d} \widehat{C}_{+}\|f\|_{\infty} \int_{0}^{t} \frac{1}{(t-s)^{1 / 2}} \mathbb{E}\left[\left|b\left(s, X^{x}\right)\right|\right] \mathrm{d} s<\infty .
\end{aligned}
$$

Therefore, from (15) and Fubini's theorem we obtain (13), which is the first representation (11).

\subsection{Gaussian two-sided bound and continuity of pdf}

In this subsection, we prove the Gaussian two-sided bound and continuity for a pdf of a solution of SDE (1) under the following sub-linear growth condition on the drift coefficient $b$. 
Assumption 3.2. We suppose that the drift coefficient $b$ satisfies the following condition : for any $\delta, t>0$, there exists $K_{t}(\delta)>0$ such that $K_{t}(\delta)$ is increasing with respect to $t$ and for all $t>0$ and $w \in C\left([0, t] ; \mathbb{R}^{d}\right)$,

$$
|b(t, w)| \leq \delta\left|w_{t}^{*}\right|+K_{t}(\delta)
$$

Remark 3.3. (i) Let $f: \mathbb{R}^{d} \rightarrow \mathbb{R}^{d}$ be a measurable function and of sub-linear growth, that is, $f$ is bounded on any compact subset of $\mathbb{R}^{d}$ and $|f(x)|=o(|x|)$ as $|x| \rightarrow \infty$, which is equivalent to the condition that for any $\delta>0$, there exists a constant $K(\delta)>0$ such that $|f(x)| \leq \delta|x|+K(\delta)$. Therefore, if $b$ satisfies Assumption 3.2, then we say that $b$ is of sub-linear growth.

(ii) Suppose that $b:[0, \infty) \times C\left([0, \infty) ; \mathbb{R}^{d}\right) \rightarrow \mathbb{R}^{d}$ satisfies the following growing condition: there exists $K>0$ and $\beta \in(0,1)$ such that

$$
|b(t, w)| \leq K\left(1+\left|w_{t}^{*}\right|^{\beta}\right), \text { for all }(t, w) \in[0, \infty) \times C\left([0, \infty) ; \mathbb{R}^{d}\right) .
$$

Then $b$ satisfies Assumption 3.2 with $K_{t}(\delta)=K\left\{1+(K / \delta)^{\beta /(1-\beta)}\right\}$ for all $t>0$. Indeed it holds that

$$
K\left(1+\left|w_{t}^{*}\right|^{\beta}\right) \leq \begin{cases}\delta\left|w_{t}^{*}\right|+K & \text { if }\left|w_{t}^{*}\right|>(K / \delta)^{1 /(1-\beta)} \\ K\left\{1+(K / \delta)^{\beta /(1-\beta)}\right\} & \text { if }\left|w_{t}^{*}\right| \leq(K / \delta)^{1 /(1-\beta)}\end{cases}
$$

Under the sub-linear growth condition on $b$, we prove a Gaussian two-sided bound and a continuity for a pdf of $X_{t}^{x}$.

Theorem 3.4. Suppose Assumption 2.1 and 3.2 hold. Let $p_{1}, p_{2}, p_{3}>1$ with $p_{1} \in\left(1, \frac{d}{d-1}\right)$ and $1 / p_{1}+1 / p_{2}+1 / p_{3}=1$.

(i) For each $(t, x) \in(0, T] \times \mathbb{R}^{d}$, the right hand side of (11) is continuous with respect to $y$, that is, $p_{t}(x, \cdot)$ has a continuous version.

(ii) There exist $C_{ \pm} \equiv C_{ \pm}\left(p_{1}\right)>0$ such that for any $(t, x) \in(0, T] \times \mathbb{R}^{d}$ and a.e. $y \in \mathbb{R}^{d}$, it holds that

$$
p_{t}(x, y) \geq \frac{C_{-} g_{2^{-1} \widehat{c}_{-} t}(x, y)}{1+\sup _{0 \leq s \leq t} \mathbb{E}\left[Z_{s}\left(1, Y^{0, x}\right)^{-p_{2}}\right]^{1 / p_{2}} \max _{i=1,2} \mathbb{E}\left[\left|b\left(s, Y^{0, x}\right)\right|^{i p_{3}}\right]^{1 / p_{3}}},
$$

and

$$
p_{t}(x, y) \leq C_{+}\left(1+\sup _{0 \leq s \leq t} \mathbb{E}\left[Z_{s}\left(1, Y^{0, x}\right)^{p_{2}}\right]^{1 / p_{2}} \max _{i=1,2} \mathbb{E}\left[\left|b\left(s, Y^{0, x}\right)\right|^{i p_{3}}\right]^{1 / p_{3}}\right) g_{p_{1} \widehat{c}_{+} t}(x, y) .
$$

(iii) Let $p_{t}(x, \cdot)$ be a continuous version of a pdf of $X_{t}^{x}$ for $(t, x) \in(0, T] \times \mathbb{R}^{d}$. For $r \in \mathbb{R}$, we define $t_{r}$ by

$$
t_{r}:=\min \left\{T, \frac{1}{2 K(b, T) \sqrt{3 \underline{a}\left(2 r^{2}-r\right) \widehat{c}_{+}}}\right\} .
$$


Then there exist $C_{ \pm}>0$ and $c_{ \pm}>0$ such that for any $(t, x, y) \in(0, T] \times \mathbb{R}^{d} \times \mathbb{R}^{d}$, it holds that if $t \in\left(0, t_{-p_{2}}\right]$, then

$$
\frac{C_{-} g_{2^{-1} \widehat{c}_{-} t}(x, y)}{\left(1+|x|^{2}\right) \exp \left(c_{-}\left(1+|x|^{2}\right) t\right)} \leq p_{t}(x, y) \leq C_{+}\left(1+|x|^{2}\right) \exp \left(c_{+}\left(1+|x|^{2}\right) t\right) g_{p_{1} \widehat{c}_{+} t}(x, y),
$$

and if $t \in\left(t_{-p_{2}}, t_{p_{2}}\right]$, then

$$
\frac{C_{-} g_{2^{-1} \widehat{c}_{-} t}(x, y)}{\left(1+|x|^{2}\right) \exp \left(\frac{|x|^{2}}{8 p_{2} \widehat{c}_{+} T}\right)} \leq p_{t}(x, y) \leq C_{+}\left(1+|x|^{2}\right) \exp \left(c_{+}\left(1+|x|^{2}\right) t\right) g_{p_{1} \widehat{c}_{+} t}(x, y),
$$

and if $t \in\left(t_{p_{2}}, T\right]$, then

$$
\frac{C_{-} g_{2^{-1} \widehat{c}_{-} t}(x, y)}{\left(1+|x|^{2}\right) \exp \left(\frac{|x|^{2}}{8 p_{2} \widehat{c}_{+} T}\right)} \leq p_{t}(x, y) \leq C_{+}\left(1+|x|^{2}\right) \exp \left(\frac{|x|^{2}}{8 p_{2} \widehat{c}_{+} T}\right) g_{p_{1} \widehat{c}_{+} t}(x, y) .
$$

Remark 3.5. Note that if $b$ is bounded, then from Theorem 3.4 (ii) and (21) below, $p_{t}(x, y)$ satisfies the Gaussian two-sided bound uniformly with respect to $x \in \mathbb{R}^{d}$, that is, there exist $C_{ \pm}>0$ and $c_{ \pm}$such that for any $x, y \in \mathbb{R}^{d}$ and $t \in(0, T]$,

$$
C_{-} g_{c_{-} t}(x, y) \leq p_{t}(x, y) \leq C_{+} g_{c_{+} t}(x, y)
$$

(see, also Theorem 2.5 in [38). However, if $b$ is of sub-linear growth, then $C_{ \pm}$in (19) might be depend on the initial value $x \in \mathbb{R}^{d}$. Note that an Ornstein-Uhlenbeck process $\mathrm{d} X_{t}=\kappa X_{t} \mathrm{~d} t+\mathrm{d} W_{t}$, $X_{0}=x$, the law of $X_{t}$ admits a pdf. However, it does not satisfies the Gaussian two-sided bound uniformly in $x \in \mathbb{R}$, (see, section 6.2 in [38).

Since $q(s, x ; t, y)$ is continuous in $y \in \mathbb{R}^{d}$ and satisfies the Gaussian two-sided bound, in order to obtain continuity and two-sided bound of $p_{t}(x, y)$, we need to consider

$$
\int_{0}^{t} \mathbb{E}\left[\left\langle\nabla_{x} q\left(s, X_{s}^{x} ; t, y\right), b\left(s, X^{x}\right)\right\rangle\right] \mathrm{d} s \quad \text { and } \quad \mathbb{E}\left[Z_{t}\left(1, Y^{0, x}\right) \mid Y_{t}^{0, x}=y\right] .
$$

We first introduce the following lemma which shows that the moment of the MaruyamaGirsanov density $Z_{t}\left(1, Y^{0, x}\right)$ is finite.

Lemma 3.6. Suppose Assumption 2.1 and 3.2 hold. Recall that $t_{r}$ is defined by (18) for $r \in \mathbb{R}$. For any $r \in \mathbb{R},(t, x) \in(0, T] \times \mathbb{R}^{d}$, it holds that

$$
\begin{aligned}
& \sup _{0 \leq s \leq t} \mathbb{E}\left[Z_{s}\left(1, Y^{0, x}\right)^{r}\right] \\
& \leq \begin{cases}1, & \text { if } 2 r^{2}-r \leq 0, t \in(0, T], \\
2^{1+d / 4} \widehat{C}_{+} \exp \left(\frac{3}{2} K(b, T)^{2} \underline{a}\left(2 r^{2}-r\right) t\left(1+|x|^{2}\right)\right), & \text { if } 2 r^{2}-r>0, t \in\left(0, t_{r}\right], \\
2^{1+d / 4}\left(\frac{T}{t_{r}}\right)^{d / 4} \widehat{C}_{+}^{1 / 2} \exp \left(\frac{3}{2} \underline{a}\left(2 r^{2}-r\right)\left|K_{T}\left(\delta_{r, T}\right)\right|^{2} t\right) \exp \left(\frac{|x|^{2}}{8 \widehat{c}_{+} T}\right), & \text { if } 2 r^{2}-r>0, t \in\left(t_{r}, T\right],\end{cases}
\end{aligned}
$$


where for $r \in \mathbb{R}$ and $t>0$,

$$
\delta_{r, t}:=\frac{1}{2 t \sqrt{3 \widehat{c}+\underline{a}\left(2 r^{2}-r\right)}} .
$$

Proof. For each $r \in \mathbb{R}$ and $s \in[0, t]$, by using Schwartz's inequality, we have

$$
\begin{aligned}
& \mathbb{E}\left[Z_{s}\left(1, Y^{0, x}\right)^{r}\right] \\
& =\mathbb{E}\left[\exp \left(r \sum_{j=1}^{d} \int_{0}^{s} \mu^{j}\left(u, Y^{0, x}\right) \mathrm{d} W_{u}^{j}-r^{2} \int_{0}^{s}\left|\mu\left(u, Y^{0, x}\right)\right|^{2} \mathrm{~d} u+\left(r^{2}-\frac{r}{2}\right) \int_{0}^{s}\left|\mu\left(u, Y^{0, x}\right)\right|^{2} \mathrm{~d} u\right)\right] \\
& \leq \mathbb{E}\left[Z_{s}\left(2 r, Y^{0, x}\right)\right]^{1 / 2} \mathbb{E}\left[\exp \left(\left(2 r^{2}-r\right) \int_{0}^{s}\left|\mu\left(u, Y^{0, x}\right)\right|^{2} \mathrm{~d} u\right)\right]^{1 / 2}
\end{aligned}
$$

From Theorem 2.3, $Z\left(2 r, Y^{0, x}\right)$ is martingale, thus $\mathbb{E}\left[Z_{s}\left(2 r, Y^{0, x}\right)\right]=1$. If $2 r^{2}-r \leq 0$, then (21) is bounded by 1 .

Now we assume that $2 r^{2}-r>0$. For $t \in\left(0, t_{r}\right]$, by using a linear growth condition on $b$, we have

$$
\sup _{0 \leq s \leq t} \mathbb{E}\left[Z_{s}\left(1, Y^{0, x}\right)^{r}\right] \leq \mathbb{E}\left[\exp \left(3 K(b, T)^{2} \underline{a}\left(2 r^{2}-r\right) t\left(1+|x|^{2}+\left|M_{t}^{x, *}\right|^{2}\right)\right)\right]^{1 / 2} .
$$

Since the map $z \mapsto \exp \left(\frac{3}{2} K(b, T)^{2} \underline{a}\left(2 r^{2}-r\right) t\left(1+|x|^{2}+|z|^{2}\right)\right)$ is a convex,

$$
U_{t}^{x}:=\exp \left(\frac{3}{2} K(b, T)^{2} \underline{a}\left(2 r^{2}-r\right) t\left(1+|x|^{2}+\left|M_{t}^{x}\right|^{2}\right)\right)
$$

is a sub-martingale, and using Doob's maximal inequality, we have

$$
\mathbb{E}\left[\exp \left(3 K(b, T)^{2} \underline{a}\left(2 r^{2}-r\right) t\left(1+|x|^{2}+\left|M_{t}^{x, *}\right|^{2}\right)\right)\right]=\mathbb{E}\left[\left|U_{t}^{x, *}\right|^{2}\right] \leq 4 \mathbb{E}\left[\left|U_{t}^{x}\right|^{2}\right] .
$$

Hence it follows from the Gaussian upper bound (8) that

$$
\begin{aligned}
\mathbb{E}\left[\left|U_{t}^{x}\right|^{2}\right] \leq & \widehat{C}_{+} \exp \left(3 K(b, T)^{2} \underline{a}\left(2 r^{2}-r\right) t\left(1+|x|^{2}\right)\right) \int_{\mathbb{R}^{d}} \exp \left(3 K(b, T)^{2} \underline{a}\left(2 r^{2}-r\right) t|y-x|^{2}\right) g_{\widehat{c}_{+} t}(x, y) \mathrm{d} y \\
= & 2^{d / 2} \widehat{C}_{+} \exp \left(3 K(b, T)^{2} \underline{a}\left(2 r^{2}-r\right) t\left(1+|x|^{2}\right)\right) \\
& \times \int_{\mathbb{R}^{d}} \exp \left(\left\{3 K(b, T)^{2} \underline{a}\left(2 r^{2}-r\right) t-\frac{1}{4 \widehat{c}_{+} t}\right\}|y-x|^{2}\right) g_{2 \widehat{c}_{+} t}(x, y) \mathrm{d} y .
\end{aligned}
$$

Therefore, since $t \leq t_{r}$ if and only if $3 K(b, T)^{2} \underline{a}\left(2 r^{2}-r\right) t-\frac{1}{4 \widehat{c}_{+} t} \leq 0$, we obtain the statement for $t \in\left(0, t_{r}\right]$.

For $t \in\left(t_{r}, T\right]$, using Assumption 2.1 (iii), Assumption 3.2 and (21), we have

$$
\sup _{0 \leq s \leq t} \mathbb{E}\left[Z_{s}\left(1, Y^{0, x}\right)^{r}\right] \leq \mathbb{E}\left[\exp \left(\left(2 r^{2}-r\right) \int_{0}^{t}\left|\mu\left(s, Y^{0, x}\right)\right|^{2} \mathrm{~d} s\right)\right]^{1 / 2}
$$




$$
\begin{aligned}
& \leq \mathbb{E}\left[\exp \left(3 \underline{a}\left(2 r^{2}-r\right) \int_{0}^{t} \delta^{2}\left(|x|^{2}+\left|M_{s}^{x, *}\right|^{2}\right)+\left|K_{T}(\delta)\right|^{2} \mathrm{~d} s\right)\right]^{1 / 2} \\
& \leq \exp \left(\frac{3}{2} \underline{a}\left(2 r^{2}-r\right)\left|K_{T}(\delta)\right|^{2} t\right) \mathbb{E}\left[\exp \left(3 \underline{a}\left(2 r^{2}-r\right) \delta^{2} t\left(|x|^{2}+\left|M_{t}^{x, *}\right|^{2}\right)\right)\right]^{1 / 2} .
\end{aligned}
$$

Note that the map $z \mapsto \exp \left(\frac{3}{2} \underline{a}\left(2 r^{2}-r\right) \delta^{2} t\left(|x|^{2}+|z|^{2}\right)\right)$ is convex, thus

$$
V_{t}^{x}:=\exp \left(\frac{3}{2} \underline{a}\left(2 r^{2}-r\right) \delta^{2} t\left(|x|^{2}+\left|M_{t}^{x}\right|^{2}\right)\right)
$$

is a sub-martingale, and using Doob's maximal inequality, we have

$$
\mathbb{E}\left[\exp \left(3 \underline{a}\left(2 r^{2}-r\right) \delta^{2} t\left(|x|^{2}+\left|M_{t}^{x, *}\right|^{2}\right)\right)\right]=\mathbb{E}\left[\left|V_{t}^{x, *}\right|^{2}\right] \leq 4 \mathbb{E}\left[\left|V_{t}^{x}\right|^{2}\right] .
$$

Recall that $M_{t}^{x}=Y_{t}^{0, x}-x$ and $Y_{t}^{0, x}$ has the pdf which satisfies the Gaussian upper bound (8), we have

$$
\begin{aligned}
\mathbb{E}\left[\left|V_{t}^{x}\right|^{2}\right] \leq & \left(\frac{T}{t_{r}}\right)^{d / 2} \widehat{C}_{+} \int_{\mathbb{R}^{d}} \exp \left(3 \underline{a}\left(2 r^{2}-r\right) \delta^{2} T\left(|x|^{2}+|y-x|^{2}\right)\right) g_{\widehat{c}_{+} T}(x, y) \mathrm{d} y \\
= & \left(\frac{2 T}{t_{r}}\right)^{d / 2} \widehat{C}_{+} \exp \left(3 \underline{a}\left(2 r^{2}-r\right) \delta^{2} T|x|^{2}\right) \\
& \times \int_{\mathbb{R}^{d}} \exp \left(\left\{3 \underline{a}\left(2 r^{2}-r\right) \delta^{2} T-\frac{1}{4 \widehat{c}_{+} T}\right\}|y-x|^{2}\right) g_{2 \widehat{c}_{+} T}(x, y) \mathrm{d} y .
\end{aligned}
$$

By choosing $\delta=\delta_{r, T}, 3 \underline{a}\left(2 r^{2}-r\right) \delta^{2} T-\frac{1}{4 \hat{c}_{+} T}=0$, thus we obtain the statement for $t \in\left(t_{r}, T\right]$.

Remark 3.7. Note that the sub-linear growth condition is necessary in order to show the $q$-th moment of $Z_{t}\left(1, Y^{0, x}\right)$, (see, e.g. Remark 3.3 in [50]).

The following lemma is useful for proving a Gaussian two-sided bound.

Lemma 3.8. Let $t \in(0, T], p>1$ and $p_{1}, p_{2}, p_{2}>1$ with $1 / p_{1}+1 / p_{2}+1 / p_{3}=1$. Suppose that Assumption 2.1 and 3.2 hold and $F:[0, \infty) \times C\left([0, \infty) ; \mathbb{R}^{d}\right) \rightarrow \mathbb{R}^{d}$ satisfies assumptions in Lemma 2.4.

(i) For any $(s, x, y) \in[0, t) \times \mathbb{R}^{d} \times \mathbb{R}^{d}$ it holds that

$$
\begin{aligned}
& \mathbb{E}\left[\left|\nabla_{x} q\left(s, X_{s}^{x} ; t, y\right)\right|\left|F\left(s, X^{x}\right)\right|\right] \\
& \leq \frac{\sqrt{d} \widehat{C}_{+} C_{0, \sigma}\left(p_{3}, F, T\right)(1+|x|) \mathbb{E}\left[Z_{t}\left(1, Y^{0, x}\right)^{p_{2}}\right]^{1 / p_{2}}}{\sqrt{t-s}} \mathbb{E}\left[\left|g_{\widehat{c}_{+}(t-s)}\left(Y_{s}^{0, x}, y\right)\right|^{p_{1}}\right]^{1 / p_{1}} .
\end{aligned}
$$

In particular, if $F$ is bounded and $p_{3}=\infty$,

$\mathbb{E}\left[\left|\nabla_{x} q\left(s, X_{s}^{x} ; t, y\right)\right|\left|F\left(s, X^{x}\right)\right|\right] \leq \frac{\sqrt{d} \widehat{C}_{+}\|F\|_{\infty}}{\sqrt{t-s}} \mathbb{E}\left[\left|g_{\widehat{c}_{+}(t-s)}\left(Y_{s}^{0, x}, y\right)\right|^{p_{1}}\right]^{1 / p_{1}} \mathbb{E}\left[Z_{t}\left(1, Y^{0, x}\right)^{p_{2}}\right]^{1 / p_{2}}$ 
(ii) For any $c>0$ and $(s, x, y) \in[0, t) \times \mathbb{R}^{d} \times \mathbb{R}^{d}$, it holds that

$$
\mathbb{E}\left[\left|g_{c(t-s)}\left(Y_{s}^{0, x}, y\right)\right|^{p}\right]^{1 / p} \leq \frac{\widehat{C}_{+}^{\frac{1}{p}}}{c^{\frac{d(p-1)}{2 p}}} \frac{\left\{p\left(c \vee \widehat{c}_{+}\right)\right\}^{\frac{d}{2}}}{\left(c \wedge \widehat{c}_{+}\right)^{\frac{d}{2 p}}}\left(\frac{t}{t-s}\right)^{\frac{d(p-1)}{2 p}} g_{p\left(c \vee \widehat{c}_{+}\right) t}(x, y) .
$$

Proof. (i). For any $s \in[0, t)$, using Theorem 2.3] Hölder's inequality and (9), we have,

$$
\begin{aligned}
& \mathbb{E}\left[\left|\nabla_{x} q\left(s, X_{s}^{x} ; t, y\right)\right|\left|F\left(s, X^{x}\right)\right|\right]=\mathbb{E}\left[\left|\nabla_{x} q\left(s, Y_{s}^{0, x} ; t, y\right)\right|\left|F\left(s, Y^{0, x}\right)\right| Z_{t}\left(1, Y^{0, x}\right)\right] \\
& \leq \frac{\sqrt{d} \widehat{C}_{+}}{\sqrt{t-s}} \mathbb{E}\left[g_{\widehat{c}_{+}(t-s)}\left(Y_{s}^{0, x}, y\right)\left|F\left(s, Y^{0, x}\right)\right| Z_{t}\left(1, Y^{0, x}\right)\right] \\
& \leq \frac{\sqrt{d} \widehat{C}_{+}}{\sqrt{t-s}} \mathbb{E}\left[\left|g_{\widehat{c}_{+}(t-s)}\left(Y_{s}^{0, x}, y\right)\right|^{p_{1}}\right]^{1 / p_{1}} \mathbb{E}\left[Z_{t}\left(1, Y^{0, x}\right)^{p_{2}}\right]^{1 / p_{2}} \mathbb{E}\left[\left|F\left(s, Y^{0, x}\right)\right|^{p_{3}}\right]^{1 / p_{3}} .
\end{aligned}
$$

By using Lemma 2.4 with $b \equiv 0$, we conclude the proof of (i).

(ii) By using the upper bounds (8) and Chapman-Kolmogorov equation, it holds that

$$
\begin{aligned}
& \mathbb{E}\left[\left|g_{c(t-s)}\left(Y_{s}^{0, x}, y\right)\right|^{p}\right]=\int_{\mathbb{R}^{d}} q(0, x ; s, z)\left|g_{c(t-s)}(z, y)\right|^{p} \mathrm{~d} z \leq \widehat{C}_{+} \int_{\mathbb{R}^{d}} g_{\widehat{c}_{+} s}(x, z)\left|g_{c(t-s)}(z, y)\right|^{p} \mathrm{~d} z \\
& \leq\left(\frac{c \vee \widehat{c}_{+}}{c \wedge \widehat{c}_{+}}\right)^{d / 2} \frac{\widehat{C}_{+}}{\{2 \pi c(t-s)\}^{\frac{d(p-1)}{2}}} \int_{\mathbb{R}^{d}} g_{\left(c \vee \widehat{c}_{+}\right) s}(x, z) g_{\left(c \vee \widehat{c}_{+}\right)(t-s)}(z, y) \mathrm{d} z \\
& =\left(\frac{c \vee \widehat{c}_{+}}{c \wedge \widehat{c}_{+}}\right)^{d / 2} \frac{\widehat{C}_{+}}{\{2 \pi c(t-s)\}^{\frac{d(p-1)}{2}}} g_{\left(c \vee \widehat{c}_{+}\right) t}(x, y),
\end{aligned}
$$

which concludes the statement.

For the proof of the Gaussian two-sided bound, we need the following lemma, which is an analogy of Lemma 2.3 in 38 .

Lemma 3.9. Let $r \in \mathbb{R}$ and $p_{1}, p_{2}, p_{3}>1$ with $p_{1} \in\left(1, \frac{d}{d-1}\right), 1 / p_{1}+1 / p_{2}+1 / p_{3}=1$. Suppose Assumption 2.1 and 3.2 hold. Then there exists $C_{r, p_{1}}>0$ such that for all $(t, x, y) \in(0, T] \times \mathbb{R}^{d} \times \mathbb{R}^{d}$,

$$
\begin{aligned}
& \sup _{0 \leq s<t} \mathbb{E}\left[q\left(s ; Y_{s}^{0, x} ; t, y\right) Z_{s}\left(1, Y^{0, x}\right)^{r}\right] \\
& \leq \widehat{C}_{+} g_{\widehat{c}_{+} t}(x, y)+C_{r, p_{1}} \sup _{0 \leq s \leq t} \mathbb{E}\left[Z_{s}\left(1, Y^{0, x}\right)^{r p_{2}}\right]^{1 / p_{2}} \max _{i=1,2} \mathbb{E}\left[b\left(s, Y^{0, x}\right)^{i p_{3}}\right]^{1 / p_{3}} g_{p_{1} \widehat{c}_{+} t}(x, y) .
\end{aligned}
$$

Proof. Let $s \in[0, t)$. By Itô's formula, $Z\left(1, Y^{x}\right)^{r}$ satisfies the following linear SDE

$$
Z_{s}\left(1, Y^{x}\right)^{r}=1+\frac{r(r-1)}{2} \int_{0}^{s}\left|\mu\left(r, Y^{0, x}\right)\right|^{2} Z_{u}\left(1, Y^{0, x}\right)^{r} \mathrm{~d} u+r \sum_{j=1}^{d} \int_{0}^{s} \mu^{j}\left(u, Y^{0, x}\right) Z_{u}\left(1, Y^{0, x}\right)^{r} \mathrm{~d} W_{u}^{j}
$$

and by using (6), we have

$$
q\left(s, Y_{s}^{0, x} ; t, y\right)=q(0, x ; t, y)+\sum_{i, j=1}^{d} \int_{0}^{s} \partial_{x_{i}} q\left(u, Y_{u}^{0, x} ; t, y\right) \sigma_{i, j}\left(u, Y_{u}^{0, x}\right) \mathrm{d} W_{u}^{j} .
$$


Hence by using integration by parts formula it holds that

$$
\begin{aligned}
q\left(s, Y_{s}^{0, x} ; t, y\right) Z_{s}\left(1, Y^{0, x}\right)^{r}= & q(0, x ; t, y)+r \int_{0}^{s}\left\langle\nabla_{x} q\left(u, Y_{u}^{0, x} ; t, y\right), b\left(u, Y^{0, x}\right)\right\rangle Z_{u}\left(1, Y^{0, x}\right)^{r} \mathrm{~d} u \\
& +\frac{r(r-1)}{2} \int_{0}^{s} q\left(u, Y_{u}^{0, x} ; t, y\right)\left|\mu\left(u, Y^{0, x}\right)\right|^{2} Z_{u}\left(1, Y^{0, x}\right)^{r} \mathrm{~d} u \\
& +M_{s}^{1}+M_{s}^{2},
\end{aligned}
$$

where

$$
\begin{aligned}
& M_{s}^{1}:=\sum_{i, j=1}^{d} \int_{0}^{s} Z_{u}\left(1, Y^{0, x}\right)^{r} \partial_{x_{i}} q\left(u, Y_{u}^{0, x} ; t, y\right) \sigma_{i, j}\left(u, Y_{u}^{0, x}\right) \mathrm{d} W_{u}^{j}, \\
& M_{s}^{2}:=r \sum_{j=1}^{d} \int_{0}^{s} q\left(u, Y_{u}^{0, x} ; t, y\right) \mu^{j}\left(u, Y^{0, x}\right) Z_{u}\left(1, Y^{0, x}\right)^{r} \mathrm{~d} W_{u}^{j} .
\end{aligned}
$$

By taking expectation, it holds that

$$
\begin{aligned}
\mathbb{E}\left[q\left(s, Y_{s}^{0, x} ; t, y\right) Z_{s}\left(1, Y^{0, x}\right)^{r}\right]= & q(0, x ; t, y)+r \mathbb{E}\left[\int_{0}^{s}\left\langle\nabla_{x} q\left(u, Y_{u}^{0, x} ; t, y\right), b\left(u, Y^{0, x}\right)\right\rangle Z_{u}\left(1, Y^{0, x}\right)^{r} \mathrm{~d} u\right] \\
& +\frac{r(r-1)}{2} \mathbb{E}\left[\int_{0}^{s} q\left(u, Y_{u}^{0, x} ; t, y\right)\left|\mu\left(u, Y^{0, x}\right)\right|^{2} Z_{u}\left(1, Y^{0, x}\right)^{r} \mathrm{~d} u\right]
\end{aligned}
$$

where we use the fact that the expectations of $M_{s}^{1}$ and $M_{s}^{2}$ are zero. Indeed, since $s \in[0, t)$, there exists $t_{0} \in[0, t)$ such that $s \leq t_{0}<t$. By using the moment estimate on $Z_{u}\left(1, Y^{0, x}\right)^{r}$ (see, Lemma 3.6), and the upper bound for $\partial_{x_{i}} q(u, x ; t, y)$ (see, (9)),

$\mathbb{E}\left[\left\langle M^{1}\right\rangle_{s}\right] \leq \frac{d \bar{a} \widehat{C}_{+}^{2}}{\left(2 \pi \widehat{c}_{+}\right)^{d}} \int_{0}^{t_{0}} \frac{\mathbb{E}\left[Z_{u}\left(1, Y^{0, x}\right)^{2 r}\right]}{(t-u)^{d+1}} \mathrm{~d} u \leq \frac{d \bar{a} \widehat{C}_{+}^{2}}{\left(2 \pi \widehat{c}_{+}\right)^{d}} \sup _{0 \leq u \leq t_{0}} \mathbb{E}\left[Z_{u}\left(1, Y^{0, x}\right)^{2 r}\right] \int_{0}^{t_{0}} \frac{1}{(t-u)^{d+1}} \mathrm{~d} u<\infty$,

and the moment estimate on $b\left(u, Y^{0, x}\right)$ (see, Lemma 2.4), the upper bound for $q(u, x ; t, y)$ (see, (8)) and Schwarz's inequality

$$
\begin{aligned}
\mathbb{E}\left[\left\langle M^{2}\right\rangle_{s}\right] & \leq \frac{r^{2} \widehat{C}_{+}^{2}}{\left(2 \pi \widehat{c}_{+}\right)^{d}} \int_{0}^{t_{0}} \frac{\mathbb{E}\left[\left|\mu\left(u, Y^{0, x}\right)\right|^{2} Z_{u}\left(1, Y^{0, x}\right)^{2 r}\right]}{(t-u)^{d}} \mathrm{~d} u \\
& \leq \frac{r^{2} \widehat{C}_{+}^{2}}{\left(2 \pi \widehat{c}_{+}\right)^{d}} \sup _{0 \leq u \leq t_{0}} \mathbb{E}\left[\left|\mu\left(u, Y^{0, x}\right)\right|^{4}\right]^{1 / 2} \sup _{0 \leq u \leq t_{0}} \mathbb{E}\left[Z_{u}\left(1, Y^{0, x}\right)^{4 r}\right]^{1 / 2} \int_{0}^{t_{0}} \frac{1}{(t-u)^{d}} \mathrm{~d} u<\infty .
\end{aligned}
$$

Hence $M^{1}=\left(M_{s}^{1}\right)_{s \in\left[0, t_{0}\right]}$ and $M^{2}=\left(M_{s}^{2}\right)_{s \in\left[0, t_{0}\right]}$ are martingale, and thus the expectations of $M_{s}^{1}$ and $M_{s}^{2}$ are zero. By using (8), Schwarz's inequality and Hölder's inequality with $1 / p_{1}+1 / p_{2}+$ $1 / p_{3}=1$, we have

$$
\begin{aligned}
& \mathbb{E}\left[q\left(s, Y_{s}^{0, x} ; t, y\right) Z_{s}\left(1, Y^{0, x}\right)^{r}\right] \\
& \leq \widehat{C}_{+} g_{\widehat{c}_{+} t}(x, y)+\int_{0}^{t} \frac{r \sqrt{d} \widehat{C}_{+}}{\sqrt{t-u}} \mathbb{E}\left[g_{\widehat{c}_{+}(t-u)}\left(Y_{u}^{0, x}, y\right)\left|b\left(u, Y^{x}\right)\right| Z_{u}\left(1, Y^{x}\right)^{r}\right] \mathrm{d} u
\end{aligned}
$$




$$
\begin{aligned}
& +\frac{r(r-1) \widehat{C}_{+} \underline{a}}{2} \int_{0}^{t} \mathbb{E}\left[g_{\widehat{c}_{+}(t-u)}\left(Y_{u}^{0, x}, y\right)\left|b\left(u, Y^{0, x}\right)\right|^{2} Z_{u}\left(1, Y^{0, x}\right)^{r}\right] \mathrm{d} u \\
\leq & \widehat{C}_{+} g_{\widehat{c}_{+} t}(x, y)+\int_{0}^{t} \frac{r \sqrt{d} \widehat{C}_{+}}{\sqrt{t-u}} \mathbb{E}\left[g_{\widehat{c}_{+}(t-u)}\left(Y_{u}^{0, x}, y\right)^{p_{1}}\right]^{1 / p_{1}} \mathbb{E}\left[Z_{u}\left(1, Y^{0, x}\right)^{r p_{2}}\right]^{1 / p_{2}} \mathbb{E}\left[\left|b\left(u, Y^{0, x}\right)\right|^{p_{3}}\right]^{1 / p_{3}} \mathrm{~d} u \\
& +\frac{r(r-1) \widehat{C}_{+} \underline{a}}{2} \int_{0}^{t} \mathbb{E}\left[g_{\widehat{c}_{+}(t-u)}\left(Y_{u}^{0, x}, y\right)^{p_{1}}\right]^{1 / p_{1}} \mathbb{E}\left[Z_{u}\left(1, Y^{0, x}\right)^{r p_{2}}\right]^{1 / p_{2}} \mathbb{E}\left[\left|b\left(u, Y^{0, x}\right)\right|^{2 p_{3}}\right]^{1 / p_{3}} \mathrm{~d} u .
\end{aligned}
$$

Therefore, it follows from Lemma 2.4 Lemma 3.6 and (23) that

$$
\begin{aligned}
& \mathbb{E}\left[q\left(s, Y_{s}^{0, x} ; t, y\right) Z_{s}\left(1, Y^{0, x}\right)^{r}\right] \\
& \leq \widehat{C}_{+} g_{\widehat{c}_{+} t}(x, y)+ C \sup _{0 \leq s \leq t}\left(\mathbb{E}\left[Z_{s}\left(1, Y^{0, x}\right)^{r p_{2}}\right]^{1 / p_{2}} \max _{i=1,2} \mathbb{E}\left[b\left(s, Y^{0, x}\right)^{i p_{3}}\right]^{1 / p_{3}}\right) \\
& \times \int_{0}^{t}\left\{\frac{1}{(t-u)^{\frac{d\left(p_{1}-1\right)}{2 p_{1}}+\frac{1}{2}}}+\frac{1}{(t-u)^{\frac{d\left(p_{1}-1\right)}{2 p_{1}}}}\right\} \mathrm{d} u g_{p_{1} \widehat{c}_{+} t}(x, y),
\end{aligned}
$$

for some $C>0$. Since $p_{1} \in\left(1, \frac{d}{d-1}\right)$ implies $\frac{d\left(p_{1}-1\right)+p_{1}}{2 p_{1}}<1$, we conclude the statement.

Proof of Theorem 3.4. (Continuity). By the construction of the fundamental solution of parabolic type PDE, $q(0, x ; t, \cdot)$ is continuous (see [15]), thus it is suffices to prove that for a given $y_{0} \in \mathbb{R}^{d}$,

$$
\int_{0}^{t} \mathbb{E}\left[\left\langle\nabla_{x} q\left(s, X_{s}^{x} ; t, y_{0}\right), b\left(s, X^{x}\right)\right\rangle\right] \mathrm{d} s=\lim _{y \rightarrow y_{0}} \int_{0}^{t} \mathbb{E}\left[\left\langle\nabla_{x} q\left(s, X_{s}^{x} ; t, y\right), b\left(s, X^{x}\right)\right\rangle\right] \mathrm{d} s .
$$

We first show that for each $s \in[0, t)$,

$$
\mathbb{E}\left[\left\langle\nabla_{x} q\left(s, X_{s}^{x} ; t, y_{0}\right), b\left(s, X^{x}\right)\right\rangle\right]=\lim _{y \rightarrow y_{0}} \mathbb{E}\left[\left\langle\nabla_{x} q\left(s, X_{s}^{x} ; t, y\right), b\left(s, X^{x}\right)\right\rangle\right] .
$$

For any $(s, y) \in[0, t) \times \mathbb{R}^{d}$, by using (9), we have

$$
\left|\left\langle\nabla_{x} q\left(s, X_{s}^{x} ; t, y\right), b\left(s, X^{x}\right)\right\rangle\right| \leq \frac{\sqrt{d} \widehat{C}_{+}\left|b\left(s, X^{x}\right)\right|}{\left(2 \pi \widehat{c}_{+}\right)^{\frac{d}{2}}(t-s)^{\frac{d+1}{2}}} .
$$

Since $\nabla_{x} q(0, x ; t, \cdot)$ is continuous (e.g., page 20 of [15]), from Lemma2.4 and dominated convergence theorem, we obtain (26).

Let $p_{1}, p_{2}, p_{3}>1$ with $p_{1} \in\left(1, \frac{d}{d-1}\right), 1 / p_{1}+1 / p_{2}+1 / p_{3}=1$. By using (22) with $F \equiv b$ and (23) in Lemma 3.8 we have

$$
\begin{aligned}
& \int_{0}^{t} \sup _{y \in \mathbb{R}^{d}} \mathbb{E}\left[\left|\left\langle\nabla_{x} q\left(s, X_{s}^{x} ; t, y\right), b\left(s, X^{x}\right)\right\rangle\right|\right] \mathrm{d} s \\
& \leq C_{p_{3}}(1+|x|) \sup _{0 \leq s \leq t} \mathbb{E}\left[Z_{s}\left(1, Y^{0, x}\right)^{p_{2}}\right]^{1 / p_{2}} \int_{0}^{t} \frac{1}{(t-s)^{\frac{d\left(p_{1}-1\right)}{2 p_{1}}+\frac{1}{2}}} \mathrm{~d} s \sup _{y \in \mathbb{R}^{d}} g_{p_{1} \widehat{c}_{+} t}(x, y)<\infty,
\end{aligned}
$$

for some $C_{p_{3}}>0$. Thus again using dominated convergence theorem, we conclude (25). 
(Upper and lower bound). The proof of upper and lower bound are based on [38] and the second representation (12). By using Schwarz's inequality, it holds that

$$
\begin{aligned}
1 & =\mathbb{E}\left[Z_{t}\left(1, Y^{0, x}\right)^{1 / 2} Z_{t}\left(1, Y^{0, x}\right)^{-1 / 2} \mid Y_{t}^{0, x}=y\right]^{2} \\
& \leq \mathbb{E}\left[Z_{t}\left(1, Y^{0, x}\right) \mid Y_{t}^{0, x}=y\right] \mathbb{E}\left[Z_{t}\left(1, Y^{0, x}\right)^{-1} \mid Y_{t}^{0, x}=y\right] \leq \infty, \text { a.e., } y \in \mathbb{R}^{d},
\end{aligned}
$$

which implies that

$$
0 \leq \frac{1}{\mathbb{E}\left[Z_{t}\left(1, Y^{0, x}\right)^{-1} \mid Y_{t}^{0, x}=y\right]} \leq \mathbb{E}\left[Z_{t}\left(1, Y^{0, x}\right) \mid Y_{t}^{0, x}=y\right] \text {, a.e., } y \in \mathbb{R}^{d} .
$$

Therefore, from (12), we have

$$
p_{t}(x, y) \geq \frac{q(0, x ; t, y)^{2}}{q(0, x ; t, y) \mathbb{E}\left[Z_{t}\left(1, Y^{0, x}\right)^{-1} \mid Y_{t}^{0, x}=y\right]} \geq 0, \text { a.e., } y \in \mathbb{R}^{d} .
$$

Now we show that for any $r \in \mathbb{R}$ and $s \in[0, t)$,

$$
q(0, x ; t, y) \mathbb{E}\left[Z_{s}\left(1, Y^{0, x}\right)^{r} \mid Y_{t}^{0, x}=y\right]=\mathbb{E}\left[q\left(s, Y_{s}^{0, x} ; t, y\right) Z_{s}\left(1, Y^{0, x}\right)^{r}\right] \text {, a.e., } y \in \mathbb{R}^{d} .
$$

It is sufficient to show that for any $A \in \mathcal{F}_{s}$,

$$
q(0, x ; t, y) \mathbb{P}\left(A \mid Y_{t}^{0, x}=y\right)=\mathbb{E}\left[q\left(s, Y_{s}^{0, x} ; t, y\right) \mathbf{1}_{A}\right] \text {, a.e., } y \in \mathbb{R}^{d} .
$$

From Theorem 1.3.3 in [27], we have for any $f \in C_{b}^{\infty}\left(\mathbb{R}^{d} ; \mathbb{R}\right)$,

$$
\int_{\mathbb{R}^{d}} f(y) \mathbb{P}\left(A \mid Y_{t}^{0, x}=y\right) q(0, x ; t, y) \mathrm{d} y=\int_{\mathbb{R}^{d}} f(y) \mathbb{P}\left(A \cap\left\{Y_{t}^{0, x} \in \mathrm{d} y\right\}\right) .
$$

Using the Markov property of $Y^{0, x}$, for any $B \in \mathcal{B}\left(\mathbb{R}^{d}\right)$,

$$
\mathbb{P}\left(A \cap\left\{Y_{t}^{0, x} \in B\right\}\right)=\mathbb{E}\left[\mathbf{1}_{A} \mathbb{P}\left(Y_{t}^{0, x} \in B \mid \mathcal{F}_{s}\right)\right]=\int_{B} \mathbb{E}\left[\mathbf{1}_{A} q\left(s, Y_{s}^{0, x} ; t, y\right)\right] \mathrm{d} y .
$$

Hence, we have

$$
\int_{\mathbb{R}^{d}} f(y) \mathbb{P}\left(A \mid Y_{t}^{0, x}=y\right) q(0, x ; t, y) \mathrm{d} y=\int_{\mathbb{R}^{d}} f(y) \mathbb{E}\left[\mathbf{1}_{A} q\left(s ; Y_{s}^{0, x} ; t, y\right)\right] \mathrm{d} y .
$$

This concludes (29).

Applying Lemma 3.9. Fatou's lemma and (28), we have for any $r \in \mathbb{R}$,

$$
\begin{aligned}
& q(0, x ; t, y) \mathbb{E}\left[Z_{t}\left(1, Y^{0, x}\right)^{r} \mid Y_{t}^{0, x}=y\right] \leq q(0, x ; t, y) \liminf _{s \rightarrow 0} \mathbb{E}\left[Z_{t-s}\left(1, Y^{0, x}\right)^{r} \mid Y_{t}^{0, x}=y\right] \\
& \leq q(0, x ; t, y) \sup _{0 \leq s<t} \mathbb{E}\left[Z_{s}\left(1, Y^{0, x}\right)^{r} \mid Y_{t}^{0, x}=y\right]=\sup _{0 \leq s<t} \mathbb{E}\left[q\left(s, Y_{s}^{0, x} ; t, y\right) Z_{s}\left(1, Y^{0, x}\right)^{r}\right]
\end{aligned}
$$




$$
\begin{aligned}
& \leq \widehat{C}_{+} g_{\widehat{c}_{+} t}(x, y)+C_{r, p_{1}} \sup _{0 \leq s \leq t} \mathbb{E}\left[Z_{s}\left(1, Y^{0, x}\right)^{r p_{2}}\right]^{1 / p_{2}} \max _{i=1,2} \mathbb{E}\left[\left|b\left(s, Y^{0, x}\right)\right|^{i p_{3}}\right]^{1 / p_{3}} g_{p_{1} \widehat{c}_{+} t}(x, y) \\
& \leq \frac{\widehat{C}_{+}+C_{r, p_{1}} \sup _{0 \leq s \leq t} \mathbb{E}\left[Z_{s}\left(1, Y^{0, x}\right)^{r p_{2}}\right]^{1 / p_{2}} \max _{i=1,2} \mathbb{E}\left[\left|b\left(s, Y^{0, x}\right)\right|^{i p_{3}}\right]^{1 / p_{3}}}{\left(2 \widehat{c}_{+} t\right)^{d / 2}}<\infty,
\end{aligned}
$$

thus, $Z_{t}\left(1, Y^{x}\right)^{r}$ is $L^{1}$ integrable with respect to the expectation $\mathbb{E}\left[\cdot \mid Y_{t}^{0, x}=y\right]$ for any $r \in \mathbb{R}$. Hence (30) with $r=1$, we have

$$
p_{t}(x, y) \leq \widehat{C}_{+} g_{\widehat{c}_{+} t}(x, y)+C_{1, p_{1}} \sup _{0 \leq s \leq t} \mathbb{E}\left[Z_{s}\left(1, Y^{0, x}\right)^{p_{2}}\right]^{1 / p_{2}} \max _{i=1,2} \mathbb{E}\left[\left|b\left(s, Y^{0, x}\right)\right|^{i p_{3}}\right]^{1 / p_{3}} g_{p_{1} \widehat{c}_{+} t}(x, y),
$$

a.e., $y \in \mathbb{R}^{d}$. Moreover, the Gaussian lower bound (8) for $q(0, x ; t, y)$, and estimations (27), (31) with $r=-1$, we obtain

$$
\begin{aligned}
p_{t}(x, y) & \geq \frac{\left(2 \pi \widehat{c}_{+} t\right)^{d / 2}}{\widehat{C}_{+}+C_{-1, p_{1}} \sup _{0 \leq s \leq t} \mathbb{E}\left[Z_{s}\left(1, Y^{0, x}\right)^{-p_{2}}\right]^{1 / p_{2}} \max _{i=1,2} \mathbb{E}\left[\left|b\left(s, Y^{0, x}\right)\right|^{i p_{3}}\right]^{1 / p_{3}}} \frac{\widehat{C}_{-}^{2} \exp \left(-\frac{|y-x|^{2}}{\widehat{c}_{-} t}\right)}{\left(2 \pi \widehat{c}_{-} t\right)^{d}} \\
& =\frac{\widehat{c}_{+}^{d / 2} \widehat{c}_{-}^{-d / 2} \widehat{C}_{-}^{2}}{\widehat{C}_{+}+C_{-1, p_{1}} \sup _{0 \leq s \leq t} \mathbb{E}\left[Z_{s}\left(1, Y^{0, x}\right)^{-p_{2}}\right]^{1 / p_{2}} \max _{i=1,2} \mathbb{E}\left[\left|b\left(s, Y^{0, x}\right)\right|^{i p_{3}}\right]^{1 / p_{3}}} g_{2^{-1} \widehat{c}_{-} t}(x, y),
\end{aligned}
$$

a.e., $y \in \mathbb{R}^{d}$. Therefore, we conclude the statement (ii). The continuity of $p_{t}(x, \cdot)$, Lemma 2.4 and Lemma 3.6 with $r= \pm p_{2}$ implies the statement (iii).

\subsection{SDEs with bounded and path-dependent drift}

The parametrix method is a useful tool for studying a fundamental solution of parabolic type partial differential equations. In this subsection, we apply the parametrix method to provide another representation formula for a pdf of solution of SDE with bounded and path-dependent drift.

Let $\widetilde{X}^{s, x}=\left(\widetilde{X}_{t}^{s, x}\right)_{t \in[s, T]}$ be a solution of the following Markovian SDE of the form

$$
\widetilde{X}_{t}^{s, x}=x+\int_{s}^{t} \widetilde{b}\left(r, \widetilde{X}_{r}^{s, x}\right) \mathrm{d} r+\int_{s}^{t} \sigma\left(r, \widetilde{X}_{r}^{s, x}\right) \mathrm{d} W_{r},
$$

where $\widetilde{b}:[0, T] \times \mathbb{R}^{d} \rightarrow \mathbb{R}^{d}$ is a bounded and measurable. Under Assumption 2.1 on $\sigma$, by using the same way as a proof of Theorem 3.1, a pdf of $\widetilde{X}_{t}^{s, x}$, denoted by $\widetilde{p}(s, x ; t, \cdot)$ satisfies

$$
\begin{aligned}
\widetilde{p}(s, x ; t, y) & =q(s, x ; t, y)+\int_{s}^{t} \mathbb{E}\left[\left\langle\nabla_{x} q\left(r, \widetilde{X}_{r}^{s, x} ; t, y\right), \widetilde{b}\left(r, \widetilde{X}_{r}^{s, x}\right)\right\rangle\right] \mathrm{d} r \\
& =q(s, x ; t, y)+\int_{s}^{t} \mathrm{~d} r \int_{\mathbb{R}^{d}} \mathrm{~d} z\left\langle\nabla_{x} q(r, z ; t, y), \widetilde{b}(r, z)\right\rangle \widetilde{p}(s, x ; r, z),
\end{aligned}
$$

which is an analogue of the parametrix method (see, page 4, (2.8) in [15] or (4.4) in [39]). Moreover, we have the following "formal" expansion holds

$$
\widetilde{p}(s, x ; t, y)=\sum_{n=0}^{\infty} q \otimes \widetilde{H}^{\otimes n}(s, x ; t, y)
$$


where $\widetilde{H}(r, x ; t, y):=\left\langle\nabla_{x} q(r, x ; t, y), \widetilde{b}(r, x)\right\rangle$ and the space and time convolution operator $\otimes$ is defined by

$$
f \otimes g(s, x ; t, y):=\int_{s}^{t} \mathrm{~d} r \int_{\mathbb{R}^{d}} \mathrm{~d} z f(s, x ; r, z) g(r, z ; t, y),
$$

and we denote $f^{\otimes 1}=f, f^{\otimes k}=f^{\otimes(k-1)} \otimes f$ and $f \otimes g^{\otimes 0}=f$.

Remark 3.10. Deck and Kruse [11 shows that if the drift $b$ is of Hölder growing condition: $\sup _{0 \leq t<T}|b(t, x)| \leq K\left(1+|x|^{\beta}\right)$ with $\beta<\alpha \leq 1$, a similar expansion converges absolutely and uniformly for $(t, x, y) \in(0, T] \times \mathbb{R}^{d} \times \mathbb{R}^{d}$.

In order to provide another representation for $p_{t}(x, \cdot)$, we first show that if $\widetilde{b}$ is bounded measurable, then the expansion (32) converges absolutely and uniformly in $x, y \in \mathbb{R}^{d}$, and $\partial_{x_{i}} \widetilde{p}(s, x ; t, y)$ exists for all $i=1, \ldots, d$.

We denote $|\widetilde{H}|(s, z ; t, y):=|\widetilde{H}(s, z ; t, y)|$.

Lemma 3.11. Suppose Assumption 2.1] holds and the drift coefficient $\widetilde{b}$ is bounded and measurable. Then it holds that

$$
|\widetilde{H}|^{\otimes n}(s, z ; t, y) \leq \frac{\left(\sqrt{d}\|\widetilde{b}\|_{\infty} \widehat{C}_{+}\right)^{n}(t-s)^{(n-2) / 2} \Gamma(1 / 2)^{n}}{\Gamma(n / 2)} g_{\widehat{c}_{+}(t-s)}(z, y), n \in \mathbb{N},
$$

and there exist $C_{+}$and $c_{+}$such that for each $k \in\{0,1\}$ and $i=1 \ldots, d$,

$$
\begin{aligned}
\sum_{n=0}^{\infty} \sup _{x, y \in \mathbb{R}}\left|\partial_{x_{i}}^{k} q\right| \otimes|\widetilde{H}|^{\otimes n}(s, x ; t, y) & \leq \frac{C_{+}}{(t-s)^{k / 2}} g_{c_{+}(t-s)}(x, y), \\
\partial_{x_{i}}^{k} \widetilde{p}(s, x ; t, y) & =\sum_{n=0}^{\infty}\left(\partial_{i}^{k} q\right) \otimes \widetilde{H}^{\otimes n}(s, x ; t, y),
\end{aligned}
$$

for all $0 \leq s<t \leq T$ and $x, y \in \mathbb{R}^{d}$.

Proof. We first show (33). For $n=1$, from (9), it holds that

$$
|\widetilde{H}|(s, z ; t, y)=|\widetilde{H}(s, z ; t, y)| \leq \frac{\sqrt{d}\|\widetilde{b}\|_{\infty} \widehat{C}_{+}}{\sqrt{t-s}} g_{\widehat{c}_{+}(t-s)}(z, y) .
$$

We assume that (33) holds for $n-1 \geq 1$, then from Chapman-Kolmogorov equation, change of variable $u=t_{1}-s$ and Lemma 5.1 with $m=1, t_{0}=t-s, a=1 / 2$ and $b=(n-3) / 2>-1$, we have

$$
\begin{aligned}
|\widetilde{H}|^{\otimes n}(s, z ; t, y) & =\int_{s}^{t} \mathrm{~d} t_{1} \int_{\mathbb{R}^{d}} \mathrm{~d} z_{1}|\widetilde{H}|^{\otimes(n-1)}\left(s, z ; t_{1}, z_{1}\right)|\widetilde{H}|\left(t_{1}, z_{1} ; t, y\right) \\
& \leq \frac{\left(\sqrt{d}\|\widetilde{b}\|_{\infty} \widehat{C}_{+}\right)^{n} \Gamma(1 / 2)^{n-1}}{\Gamma((n-1) / 2)} \int_{s}^{t} \mathrm{~d} t_{1} \frac{\left(t_{1}-s\right)^{(n-1-2) / 2}}{\left(t-t_{1}\right)^{1 / 2}} \int_{\mathbb{R}^{d}} \mathrm{~d} z_{1} g_{\widehat{c}_{+}\left(t_{1}-s\right)}\left(z_{1}, z\right) g_{\widehat{c}_{+}\left(t-t_{1}\right)}\left(y, z_{1}\right) \\
& =\frac{\left(\sqrt{d}\|\widetilde{b}\|_{\infty} \widehat{C}_{+}\right)^{n} \Gamma(1 / 2)^{n-1}}{\Gamma((n-1) / 2)} g_{\widehat{c}_{+}(t-s)}(z, y) \int_{0}^{t-s} \frac{u^{(n-3) / 2}}{(t-s-u)^{1 / 2}} \mathrm{~d} u
\end{aligned}
$$




$$
=\frac{\left(\sqrt{d}\|\widetilde{b}\|_{\infty} \widehat{C}_{+}\right)^{n}(t-s)^{(n-2) / 2} \Gamma(1 / 2)^{n}}{\Gamma(n / 2)} g_{\widehat{c}_{+}(t-s)}(z, y) .
$$

Hence (33) holds for every $n \in \mathbb{N}$.

Now we consider the expansion (35) and upper bound (34). By using (9) and (33), ChapmanKolmogorov equation, change of variable $u=t-t_{1}$ and Lemma 5.1 with $m=1, t_{0}=t-s, a=k / 2$ and $b=(n-2) / 2>-1$, we have for each $n \in \mathbb{N}, k \in\{0,1\}$ and $i=1 \ldots, d$,

$$
\begin{aligned}
\left|\partial_{x_{i}}^{k} q\right| \otimes|\widetilde{H}|^{\otimes n}(s, x ; t, y) & =\int_{s}^{t} \mathrm{~d} t_{1} \int_{\mathbb{R}^{d}} \mathrm{~d} z_{1}\left|\partial_{x_{i}}^{k} q\left(s, x ; t_{1}, z_{1}\right)\right||\widetilde{H}|^{\otimes n}\left(t_{1}, z_{1} ; t, y\right) \\
& \leq \frac{\left(\sqrt{d}\|\widetilde{b}\|_{\infty}\right)^{n} \widehat{C}_{+}^{n+1} \Gamma(1 / 2)^{n}}{\Gamma(n / 2)} \int_{s}^{t} \frac{\left(t-t_{1}\right)^{(n-2) / 2}}{\left(t_{1}-s\right)^{k / 2}} \mathrm{~d} t_{1} \cdot g_{\widehat{c}_{+}(t-s)}(x, y) \\
& =\frac{\left(\sqrt{d}\|\widetilde{b}\|_{\infty}\right)^{n} \widehat{C}_{+}^{n+1} \Gamma(1 / 2)^{n}}{\Gamma(n / 2)} \int_{0}^{t-s} \frac{u^{(n-2) / 2}}{(t-s-u)^{k / 2}} \mathrm{~d} u \cdot g_{\widehat{c}_{+}(t-s)}(x, y) \\
& =\frac{\left(\sqrt{d}\|\widetilde{b}\|_{\infty}\right)^{n} \widehat{C}_{+}^{n+1}(t-s)^{(n-k) / 2} \Gamma(1 / 2)^{n} \Gamma(1-k / 2)}{\Gamma((n+2-k) / 2)} g_{\widehat{c}_{+}(t-s)}(x, y) \\
& \leq \frac{\left(\sqrt{d}\|\widetilde{b}\|_{\infty}\right)^{n} \widehat{C}_{+}^{n+1} T^{n / 2} \Gamma(1 / 2)^{n} \Gamma(1-k / 2)}{\Gamma((n+2-k) / 2)} \frac{g_{\widehat{c}_{+}(t-s)}(x, y)}{(t-s)^{k / 2}} .
\end{aligned}
$$

Hence we have

$$
\sum_{n=0}^{\infty} \sup _{x, y \in \mathbb{R}}\left|\partial_{x_{i}}^{k} q\right| \otimes|\widetilde{H}|^{\otimes n}(s, x ; t, y)<\infty,
$$

which concludes the statement.

We obtain the following representation on $p_{t}(x, y)$.

Theorem 3.12. Suppose Assumption 2.1 holds and $b, \widetilde{b}$ are bounded. Then for any $(t, x, y) \in$ $(0, T] \times \mathbb{R}^{d} \times \mathbb{R}^{d}$, it holds that

$$
p_{t}(x, y)=\widetilde{p}(0, x ; t, y)+\int_{0}^{t} \mathbb{E}\left[\left\langle\nabla_{x} \widetilde{p}\left(s, X_{s}^{x} ; t, y\right), b\left(s, X^{x}\right)-\widetilde{b}\left(s, X_{s}^{x}\right)\right\rangle\right] \mathrm{d} s .
$$

Proof. We define $\Delta(0, x ; t, y):=p_{t}(x, y)-\widetilde{p}(0, x ; t, y)$ and

$$
\Lambda(0, x ; t, y):=\int_{0}^{t} \mathbb{E}\left[\left\langle\nabla_{x} \widetilde{p}\left(s, X_{s}^{x} ; t, y\right), b\left(s, X^{x}\right)-\widetilde{b}\left(s, X_{s}^{x}\right)\right\rangle\right] \mathrm{d} s .
$$

Then we show $\Delta(0, x ; t, y)=\Lambda(0, x ; t, y)$ for all $(t, x, y) \in(0, T] \times \mathbb{R}^{d} \times \mathbb{R}^{d}$.

We first compute $\Delta(0, x ; t, y)$. It follows from Theorem 3.1 that

$$
\widetilde{p}(0, x ; t, y)=q(0, x ; t, y)+\int_{0}^{t} \mathrm{~d} s \int_{\mathbb{R}^{d}} \mathrm{~d} z\left\langle\nabla_{x} q(s, z ; t, y), \widetilde{b}(s, z)\right\rangle \widetilde{p}(0, x ; s, z) .
$$


Hence $\Delta(0, x ; t, y)$ satisfies the following linear equation,

$$
\Delta(0, x ; t, y)=\bar{\Delta}(0, x ; t, y)+\Delta \otimes \widetilde{H}(0, x ; t, y),
$$

where the space and time convolution operator $\otimes$ is defined above and

$$
\bar{\Delta}(0, x ; t, y):=\int_{0}^{t} \mathbb{E}\left[\left\langle\nabla_{x} q\left(s, X_{s}^{x} ; t, y\right), b\left(s, X^{x}\right)-\widetilde{b}\left(s, X_{s}^{x}\right)\right\rangle\right] \mathrm{d} s .
$$

Since (38) is a linear equation, we have for any $N \in \mathbb{N}$,

$$
\begin{aligned}
\bar{\Delta}(0, x ; t, y) & =\bar{\Delta}(0, x ; t, y)+\bar{\Delta} \otimes \widetilde{H}(0, x ; t, y)+\Delta \otimes \widetilde{H}^{\otimes 2}(0, x ; t, y) \\
& =\sum_{n=0}^{N-1} \bar{\Delta} \otimes \widetilde{H}^{\otimes n}(0, x ; t, y)+\Delta \otimes \widetilde{H}^{\otimes N}(0, x ; t, y) .
\end{aligned}
$$

Now we estimate the upper bound of $\left|\Delta \otimes \widetilde{H}^{\otimes N}(0, x ; t, y)\right|$. By using (33), the Gaussian upper bound (19) for $p_{t}(x, y)$ and $\widetilde{p}(0, x ; t, y)$ and Chapman-Kolmogorov equation, we have

$$
\begin{aligned}
\left|\Delta \otimes \widetilde{H}^{\otimes N}(0, x ; t, y)\right| & \leq \int_{0}^{t} \mathrm{~d} s \int_{\mathbb{R}^{d}} \mathrm{~d} z\left\{p_{t}(x, Y)+\widehat{p}(0, x ; t, y)\right\}|\widetilde{H}|^{\otimes N}(s, z ; t, y) \\
& \leq \frac{2 C_{+} \widehat{C}_{+}^{N} T^{N / 2} \Gamma(1 / 2)^{N}}{\Gamma(N / 2)} g_{c_{+} t}(x, y) \rightarrow 0,
\end{aligned}
$$

as $N \rightarrow \infty$. Hence, we obtain that $\Delta(0, x ; t, y)$ satisfies the expansion 1

$$
\Delta(0, x ; t, y)=\sum_{n=0}^{\infty} \bar{\Delta} \otimes \widetilde{H}^{\otimes n}(0, x ; t, y) .
$$

Now we prove $\Lambda(0, x ; t, y)=\Delta(0, x ; t, y)$. Recall that $\partial_{x_{i}} \widetilde{p}(s, x ; t, y)$ satisfies the expansion (35). In order to use Fubini's Theorem, we prove that the following integral is finite

$$
\begin{aligned}
& |\Lambda|(0, x ; t, y) \\
& :=\sum_{n=0}^{\infty} \sum_{i=1}^{d} \int_{0}^{t} \mathrm{~d} s \int_{s}^{t} \mathrm{~d} r \int_{\mathbb{R}^{d}} \mathrm{~d} w \mathbb{E}\left[\left|\left(\partial_{i} q\right)\left(s, X_{s}^{x} ; r, w\right)\right|\left|b^{i}\left(s, X^{x}\right)-\widetilde{b}^{i}\left(s, X_{s}^{x}\right)\right|\right]|\widetilde{H}|^{\otimes n}(r, w ; t, y) .
\end{aligned}
$$

Since $b$ and $\widetilde{b}$ are bounded, by using (36) and Gaussian upper bound (19) for $p_{s}(x, \cdot)$, there exists $C>0$ such that

$$
\begin{aligned}
& |\Lambda|(0, x ; t, y) \\
& \leq\left(\|b\|_{\infty} \vee\|\widetilde{b}\|_{\infty}\right) \sum_{n=0}^{\infty} \sum_{i=1}^{d} \int_{0}^{t} \mathrm{~d} s \int_{s}^{t} \mathrm{~d} r \int_{\mathbb{R}^{d}} \mathrm{~d} w \int_{\mathbb{R}^{d}} \mathrm{~d} v\left|\left(\partial_{i} q\right)(s, v ; r, w) \| \widetilde{H}\right|^{\otimes n}(r, w ; t, y) p_{s}(x, v)
\end{aligned}
$$

\footnotetext{
${ }^{1}$ The procedure to obtain this expansion is called the parametrix method, and $\bar{\Delta}(0, x ; t, y)$ is called the parametrix (see, page $4,(2.8)$ in [15] or (4.4) in [39]).
} 


$$
\leq \sum_{n=0}^{\infty} \frac{C^{n}}{\Gamma((n+1) / 2)} \int_{0}^{t} \mathrm{~d} s \frac{1}{\sqrt{t-s}} \int_{\mathbb{R}^{d}} \mathrm{~d} v g_{\widehat{c}_{+}(t-s)}(v, y) g_{\widehat{c}_{+} s}(x, v)<\infty .
$$

Therefore, using Fubini's Theorem, we have

$$
\begin{aligned}
& \Lambda(0, x ; t, y) \\
& =\sum_{n=0}^{\infty} \sum_{i=1}^{d} \int_{0}^{t} \mathrm{~d} s \int_{s}^{t} \mathrm{~d} r \int_{\mathbb{R}^{d}} \mathrm{~d} w \mathbb{E}\left[\left(\partial_{i} q\right)\left(s, X_{s}^{x} ; r, w\right)\left\{b^{i}\left(s, X^{x}\right)-\widetilde{b}^{i}\left(s, X_{s}^{x}\right)\right\}\right] \widetilde{H}^{\otimes n}(r, w ; t, y) \\
& =\sum_{n=0}^{\infty} \int_{0}^{t} \mathrm{~d} r \int_{\mathbb{R}^{d}} \mathrm{~d} w \int_{0}^{r} \mathrm{~d} s \mathbb{E}\left[\left\langle\nabla_{x} q\left(s, X_{s}^{x} ; r, w\right), b\left(s, X^{x}\right)-\widetilde{b}\left(s, X_{s}^{x}\right)\right\rangle\right] \widetilde{H}^{\otimes n}(r, w ; t, y) \\
& =\sum_{n=0}^{\infty} \int_{0}^{t} \mathrm{~d} r \int_{\mathbb{R}^{d}} \mathrm{~d} w \bar{\Delta}(0, x ; r, w) \widetilde{H}^{\otimes n}(r, w ; t, y)=\sum_{n=0}^{\infty} \bar{\Delta} \otimes \widetilde{H}^{\otimes n}(0, x ; t, y)=\Delta(0, x ; t, y),
\end{aligned}
$$

which concludes the proof.

\subsection{Sharp bounds for a pdf of Brownian motion with bounded drift}

Inspired by [54, 55], we consider a sharp two-sided bound for a Brownian motion with pathdependent and bounded drift coefficient of the form

$$
X_{t}^{x}=x+\int_{0}^{t} b\left(s, X^{x}\right) \mathrm{d} s+W_{t}, x \in \mathbb{R}^{d}, t \in[0, T],
$$

by using representation (37) and bang-bang diffusion processes.

We define a $d$-dimensional bang-bang diffusion process $Y^{x, \alpha, \beta}=\left(Y_{t}^{x, \alpha, \beta}\right)_{t \in[0, T]}$ with parameter $\alpha=\left(\alpha_{1}, \ldots, \alpha_{d}\right)^{\top}, \beta=\left(\beta_{1}, \ldots, \beta_{d}\right)^{\top} \in \mathbb{R}^{d}$, which satisfies the following SDE

$$
Y_{t}^{x, \alpha, \beta}=x+\int_{0}^{t} \beta \operatorname{sgn}\left(\alpha-Y_{s}^{x, \alpha, \beta}\right) \mathrm{d} s+W_{t},
$$

where $\beta \operatorname{sgn}(x):=\left(\beta_{1} \operatorname{sgn}\left(x_{1}\right), \ldots, \beta_{d} \operatorname{sgn}\left(x_{d}\right)\right)^{\top}$, for each $x \in \mathbb{R}^{d}$. Then it follows from Theorem 2 in [54] (see also (6.5.14) in [28]) that for any $t \in(0, T], Y_{t}^{x, \alpha, \beta}$ admits a pdf, denoted by $q_{t}^{\alpha, \beta}(x, \cdot)$ which satisfies

$$
q_{t}^{\alpha, \beta}(x, \alpha)=\prod_{i=1}^{d} \frac{2}{\sqrt{2 \pi t}} \int_{\left|x_{i}-\alpha_{i}\right| / \sqrt{t}}^{\infty} z_{i} \exp \left(-\frac{\left(z_{i}-\beta_{i} \sqrt{t}\right)^{2}}{2}\right) \mathrm{d} z_{i} .
$$

Theorem 3.13. Suppose Assumption 2.1 holds and the drift coefficient $b$ is bounded. Then a pdf of a solution of (39), denoted by $p_{t}(x, \cdot)$ satisfies the following two-sided estimates: for any $(t, x, y) \in[0, T] \times \mathbb{R}^{d} \times \mathbb{R}^{d}$,

$$
q_{t}^{y,-\|b\|_{\infty}}(x, y) \leq p_{t}(x, y) \leq q_{t}^{y,\|b\|_{\infty}}(x, y) .
$$


Proof. Let $x, y \in \mathbb{R}^{d}$ be fixed. Using Theorem 3.12 with $\widetilde{p}=q^{y, \pm\|b\|_{\infty}}$, we have

$$
p_{t}(x, y)-q_{t}^{y, \pm\|b\|_{\infty}}(x, y)=\int_{0}^{t} \mathbb{E}\left[\left\langle\nabla_{x} q_{t-s}^{y, \pm\|b\|_{\infty}}\left(X_{s}^{x}, y\right), b\left(s, X^{x}\right)-\left( \pm\|b\|_{\infty}\right) \operatorname{sgn}\left(y-X_{s}^{x}\right)\right\rangle\right] \mathrm{d} s .
$$

On the other hand, it holds that for any $s \in[0, t), z \in \mathbb{R}^{d}$ and $w \in C\left([0, \infty) ; \mathbb{R}^{d}\right)$,

$$
\begin{gathered}
\partial_{z_{i}} q_{t-s}^{y,\|b\|_{\infty}}(z, y)\left(b^{i}(s, w)-\|b\|_{\infty} \operatorname{sgn}\left(y_{i}-z_{i}\right)\right) \leq 0, \\
\partial_{z_{i}} q_{t-s}^{y,-\|b\|_{\infty}}(z, y)\left(b^{i}(s, w)+\|b\|_{\infty} \operatorname{sgn}\left(y_{i}-z_{i}\right)\right) \geq 0,
\end{gathered}
$$

thus we conclude the statement.

\subsection{Comparison property of pdfs}

In this subsection, we consider a comparison property of pdfs. Let $X^{x}$ and $\widehat{X}^{x}$ be a solution of path-dependent SDE (11) with drift coefficient $b$ and $\widehat{b}$, respectively. We denote by $p_{t}(x, \cdot)$ and $\widehat{p}_{t}(x, \cdot)$ pdf of $X_{t}^{x}$ and $\widehat{X}_{t}^{x}$ for $t \in(0, T]$, respectively. Then we have the following comparison property of $p_{t}(x, \cdot)$ and $\widehat{p}_{t}(x, \cdot)$.

Theorem 3.14. Suppose Assumption 2.1 and 3.2 hold for $b, \widehat{b}$ and $\sigma$. Let $p_{1}, p_{2}, p_{3}>1$ with $p_{1} \in\left(1, \frac{d}{d-1}\right)$ and $1 / p_{1}+1 / p_{2}+1 / p_{3}=1$.

(i) There exists $C_{+} \equiv C_{+}\left(p_{1}\right)>0$ such that for any $(t, x) \in(0, T] \times \mathbb{R}^{d}$ and a.e. $y \in \mathbb{R}^{d}$, it holds that

$$
\begin{aligned}
& \left|p_{t}(x, y)-\widehat{p}_{t}(x, y)\right| \\
& \leq C_{+} \sup _{0 \leq s \leq t}\left\{\mathbb{E}\left[Z_{s}\left(1, Y^{0, x}\right)^{2 p_{2}}\right]^{\frac{1}{2 p_{2}}}+\mathbb{E}\left[\widehat{Z}_{s}\left(1, Y^{0, x}\right)^{2 p_{2}}\right]^{\frac{1}{2 p_{2}}}\right\} \mathbb{E}\left[\widehat{b}\left(s, Y^{0, x}\right)^{2 p_{2}}\right]^{\frac{1}{2 p_{2}}} \\
& \quad \times \mathbb{E}\left[\left|b\left(s, Y^{0, x}\right)+\widehat{b}\left(s, Y^{0, x}\right)\right|^{2 p_{3}}\right]^{\frac{1}{2 p_{3}}} \mathbb{E}\left[\left|b\left(s, Y^{0, x}\right)-\widehat{b}\left(s, Y^{0, x}\right)\right|^{2 p_{3}}\right]^{\frac{1}{2 p_{3}}} g_{p_{1} \widehat{c}_{+} t}(x, y) .
\end{aligned}
$$

(ii) Recall that $t_{p_{2}}$ is defined by (18). There exist $C_{+}>0$ and $c_{+}>0$ such that for any $(t, x) \in$ $(0, T] \times \mathbb{R}^{d}$ and a.e. $y \in \mathbb{R}^{d}$, it holds that if $t \in\left(0, t_{2 p_{2}}\right]$, then

$$
\begin{aligned}
& \left|p_{t}(x, y)-\widehat{p}_{t}(x, y)\right| \\
& \leq C_{+} \exp \left(c_{+}\left(1+|x|^{2}\right) t\right)(1+|x|)^{2} \sup _{0 \leq s \leq t} \mathbb{E}\left[\left|b\left(s, Y^{0, x}\right)-\widehat{b}\left(s, Y^{0, x}\right)\right|^{2 p_{3}}\right]^{\frac{1}{2 p_{3}}} g_{p_{1} \widehat{c}_{+} t}(x, y),
\end{aligned}
$$

and if $t \in\left(t_{2 p_{2}}, T\right]$, then

$$
\begin{aligned}
& \left|p_{t}(x, y)-\widehat{p}_{t}(x, y)\right| \\
& \leq C_{+} \exp \left(\frac{|x|^{2}}{16 \widehat{c}_{+} p_{2} T}\right)(1+|x|)^{2} \sup _{0 \leq s \leq t} \mathbb{E}\left[\left|b\left(s, Y^{0, x}\right)-\widehat{b}\left(s, Y^{0, x}\right)\right|^{2 p_{3}}\right]^{\frac{1}{2 p_{3}}} g_{p_{1} \widehat{c}_{+} t}(x, y) .
\end{aligned}
$$


Proof. By using Theorem 3.1 and (10), we have

$$
\left|p_{t}(x, y)-\widehat{p}_{t}(x, y)\right| \leq I_{t}^{(1)}(x, y)+I_{t}^{(2)}(x, y),
$$

where $I_{t}^{(1)}(x, y)$ and $I_{t}^{(2)}(x, y)$ are defined by

$$
\begin{aligned}
& I_{t}^{(1)}(x, y):=\int_{0}^{t} \mathbb{E}\left[\left|\nabla_{x} q\left(s, X_{s}^{0, x} ; t, y\right)\right|\left|b\left(s, X^{0, x}\right)-\widehat{b}\left(s, X^{0, x}\right)\right|\right] \mathrm{d} s, \\
& I_{t}^{(2)}(x, y):=\int_{0}^{t} \mathbb{E}\left[\left|\nabla_{x} q\left(s, Y_{s}^{0, x} ; t, y\right)\right|\left|Z_{s}\left(1, Y^{0, x}\right)-\widehat{Z}_{s}\left(1, Y^{0, x}\right)\right|\left|\widehat{b}\left(s, Y_{s}^{0, x}\right)\right|\right] \mathrm{d} s,
\end{aligned}
$$

and for $q \in \mathbb{R}, \widehat{Z}\left(q, Y^{0, x}\right)=\left(\widehat{Z}_{t}\left(q, Y^{0, x}\right)\right)_{t \in[0, T]}$ is a martingale defined by

$$
\begin{aligned}
\widehat{Z}_{t}\left(q, Y^{0, x}\right) & :=\exp \left(\sum_{j=1}^{d} \int_{0}^{t} q \widehat{\mu}^{j}\left(s, Y^{0, x}\right) \mathrm{d} W_{s}^{j}-\frac{1}{2} \int_{0}^{t}\left|q \widehat{\mu}\left(s, Y^{0, x}\right)\right|^{2} \mathrm{~d} s\right), \\
\widehat{\mu}(t, w) & :=\sigma\left(t, w_{t}\right)^{-1} \widehat{b}(t, w),(t, w) \in[0, T] \times C\left([0, T] ; \mathbb{R}^{d}\right) .
\end{aligned}
$$

By using the inequality (24) and Lemma 3.8, we have

$$
\begin{aligned}
& I_{t}^{(1)}(x, y) \\
& \leq \int_{0}^{t} \frac{\sqrt{d} \widehat{C}_{+}}{(t-s)^{1 / 2}} \mathbb{E}\left[\left|g_{\widehat{c}_{+} t}\left(Y_{s}^{0, x}, y\right)\right|^{p_{1}}\right]^{1 / p_{1}} \mathbb{E}\left[\left|Z_{s}\left(1, Y^{0, x}\right)\right|^{p_{2}}\right]^{1 / p_{2}} \mathbb{E}\left[\left|b\left(s, Y_{s}^{0, x}\right)-\widehat{b}\left(s, Y_{s}^{0, x}\right)\right|^{p_{3}}\right]^{1 / p_{3}} \mathrm{~d} s \\
& \leq C_{p_{1}} \sup _{0 \leq s \leq t} \mathbb{E}\left[Z_{s}\left(1, Y^{0, x}\right)^{p_{2}}\right]^{1 / p_{2}} \mathbb{E}\left[\left|b\left(s, Y^{0, x}\right)-\widehat{b}\left(s, Y^{0, x}\right)\right|^{p_{3}}\right]^{1 / p_{3}} g_{\widehat{c}_{+} t}(x, y) \int_{0}^{t} \frac{1}{(t-s)^{\frac{d\left(p_{1}-1\right)}{2 p_{1}}+\frac{1}{2}}} \mathrm{~d} s \\
& \leq C_{p_{1}}^{\prime} \sup _{0 \leq s \leq t} \mathbb{E}\left[Z_{s}\left(1, Y^{0, x}\right)^{p_{2}}\right]^{1 / p_{2}} \mathbb{E}\left[\left|b\left(s, Y^{0, x}\right)-\widehat{b}\left(s, Y^{0, x}\right)\right|^{p_{3}}\right]^{1 / p_{3}} g_{\widehat{c}_{+} t}(x, y),
\end{aligned}
$$

for some $C_{p_{1}}, C_{p_{1}}^{\prime}>0$. For the second term of (41), by using Hölder's inequality, the elementary estimate $\left|e^{x}-e^{y}\right| \leq\left(e^{x}+e^{y}\right)|x-y|$, Minkowski's inequality and Lemma 3.8, we have

$$
\begin{aligned}
& I_{t}^{(2)}(x, y) \\
& \leq \int_{0}^{t} \mathbb{E}\left[\left|\nabla_{x} q\left(s, Y_{s}^{0, x} ; t, y\right)\right|^{p_{1}}\right]^{1 / p_{1}} \mathbb{E}\left[\left|Z_{s}\left(1, Y^{0, x}\right)+\widehat{Z}_{s}\left(1, Y^{0, x}\right)\right|^{2 p_{2}}\right]^{1 / 2 p_{2}} \mathbb{E}\left[\left|\widehat{b}\left(s, Y^{0, x}\right)\right|^{2 p_{2}}\right]^{1 / 2 p_{2}} \\
& \quad \times \mathbb{E}\left[\left.\left|\sum_{j=1}^{d} \int_{0}^{s} \mu^{j}\left(u, Y^{0, x}\right)-\widehat{\mu}^{j}\left(u, Y^{0, x}\right) \mathrm{d} W_{u}^{j}-\frac{1}{2} \int_{0}^{s}\right| \mu\left(u, Y^{0, x}\right)\right|^{2}-\left.\left|\widehat{\mu}\left(u, Y^{0, x}\right)\right|^{2} \mathrm{~d} u\right|^{p_{3}}\right]^{1 / p_{3}} \mathrm{~d} s \\
& \leq C_{p_{1}} g_{\widehat{c}_{+} t}(x, y) \sup _{0 \leq s \leq t}\left\{\mathbb{E}\left[Z_{s}\left(1, Y^{0, x}\right)^{2 p_{2}}\right]^{1 / 2 p_{2}}+\mathbb{E}\left[\widehat{Z}_{s}\left(1, Y^{0, x}\right)^{2 p_{2}}\right]^{1 / 2 p_{2}}\right\} \mathbb{E}\left[\left|\widehat{b}\left(s, Y_{s}^{0, x}\right)\right|^{2 p_{2}}\right]^{1 / 2 p_{2}} \\
& \quad \times\left\{\mathbb{E}\left[\left|\sum_{j=1}^{d} \int_{0}^{s} \mu^{j}\left(u, Y^{0, x}\right)-\widehat{\mu}^{j}\left(u, Y^{0, x}\right) \mathrm{d} W_{u}^{j}\right|^{p_{3}}\right]^{1 / p_{3}}+\mathbb{E}\left[\left.\left|\int_{0}^{s}\right| \mu\left(u, Y^{0, x}\right)\right|^{2}-\left.\left|\widehat{\mu}\left(u, Y^{0, x}\right)\right|^{2} \mathrm{~d} u\right|^{p_{3}}\right]^{1 / p_{3}}\right\} .
\end{aligned}
$$


From Burkholder-Davis-Gundy's inequality, Schwarz's inequality and Assumption 2.1, we obtain

$$
\begin{aligned}
& I_{t}^{(2)}(x, y) \\
& \leq C_{p_{1}} g_{\widehat{c}_{+} t}(x, y) \sup _{0 \leq s \leq t}\left\{\mathbb{E}\left[Z_{s}\left(1, Y^{0, x}\right)^{2 p_{2}}\right]^{1 / 2 p_{2}}+\mathbb{E}\left[\widehat{Z}_{s}\left(1, Y^{0, x}\right)^{2 p_{2}}\right]^{1 / 2 p_{2}}\right\} \mathbb{E}\left[\widehat{b}\left(s, Y^{0, x}\right)^{2 p_{2}}\right]^{1 / 2 p_{2}} \\
& \quad \times \mathbb{E}\left[\left|b\left(s, Y^{0, x}\right)+\widehat{b}\left(s, Y^{0, x}\right)\right|^{2 p_{3}}\right]^{2 p_{3}} \mathbb{E}\left[\left|b\left(s, Y^{0, x}\right)-\widehat{b}\left(s, Y^{0, x}\right)\right|^{2 p_{3}}\right]^{1 / 2 p_{3}},
\end{aligned}
$$

for some $C_{p_{1}}>0$. Hence we conclude (40). Lemma 2.4 and Lemma 3.6 imply the statement (ii).

\subsection{Application to an Euler-Maruyama type scheme}

In this subsection, we consider an Euler-Maruyama type scheme for Brownian motion with pathdependent drift coefficient of the form

$$
\mathrm{d} X_{t}^{x}=\nu\left(A_{t}\left(X^{x}\right)\right) \mathrm{d} t+\sigma \mathrm{d} W_{t}, t \in[0, T], X_{0}=x \in \mathbb{R}^{d},
$$

where $\nu: \mathbb{S}:=[0, \infty) \times \mathbb{R}^{d} \times[0, \infty) \times\left(\mathbb{R}^{d}\right)^{\mathbb{N}} \times \mathbb{R}^{\ell} \rightarrow \mathbb{R}^{d}$ and $A_{t}: C\left([0, \infty) ; \mathbb{R}^{d}\right) \rightarrow \mathbb{S}$ is defined by (44), that is,

$$
A_{t}(w)=\left(t, w_{t}, \max _{0 \leq s \leq t} \zeta\left(s, w_{s}\right),\left\{w_{\tau_{i}(t)}\right\}_{i \in \mathbb{N}}, \int_{0}^{t} c\left(s, w_{s}\right) \mathrm{d} s,\right) \in \mathbb{S}, w \in C\left([0, \infty) ; \mathbb{R}^{d}\right),
$$

for some measurable functions $\zeta:[0, \infty) \times \mathbb{R}^{d} \rightarrow[0, \infty)$ and $c:[0, T] \times \mathbb{R}^{d} \rightarrow \mathbb{R}^{\ell}$.

Let us define an Euler-Maruyama type scheme $X^{(x, n, m)}=\left(X_{t}^{x, n, m}\right)_{t \in[0, T]}$ for SDE (42) for $n, m \in \mathbb{N}$ as follows:

$$
\mathrm{d} X_{t}^{(x, n, m)}=\nu\left(A_{t}^{(n, m)}\left(X^{(x, n, m)}\right)\right) \mathrm{d} t+\sigma \mathrm{d} W_{t}, t \in[0, T], X_{0}^{(x, n, m)}=x \in \mathbb{R}^{d},
$$

where $A_{t}^{(n, m)}(w)$ for $w \in C\left([0, T] ; \mathbb{R}^{d}\right)$ is defined by

$A_{t}^{(n, m)}(w):=\left(\eta_{n}(t), w_{\eta_{n}(t)}, \max _{0 \leq s \leq t} \zeta\left(\eta_{n}(s), w_{\eta_{n}(s)}\right),\left\{\widehat{w}_{\eta_{n}\left(\tau_{i}(t)\right)}^{(m)}\right\}_{i \in \mathbb{N}}, \int_{0}^{\eta_{n}(t)} c\left(\eta_{n}(s), w_{\eta_{n}(s)}\right) \mathrm{d} s,\right) \in \mathbb{S}$,

where $\eta_{n}(t)=k T / n$, if $t \in[k T / n,(k+1) T / n)$ and $\widehat{w}_{\eta_{n}\left(\tau_{i}(t)\right)}^{(m)}$ is defined by

$$
\widehat{w}_{\eta_{n}\left(\tau_{i}(t)\right)}^{(m)}:= \begin{cases}w_{\eta_{n}\left(\tau_{i}(t)\right)} & \text { if, } i \in\{1, \ldots, m\} \\ 0 & \text { if, } i \in\{m+1, m+2, \ldots\},\end{cases}
$$

Assumption 3.15. (i) $\nu$ is measurable and satisfies that there exists $\theta:=\left\{\theta_{i}\right\}_{i \in \mathbb{N}} \in[0, \infty)^{\mathbb{N}}$ with $\|\theta\|_{\ell_{1}}=\sum_{i \in \mathbb{N}} \theta_{i}<\infty$, and for any $\delta, t>0$, there exists $K_{t}(\delta)>0$ such that $K_{t}(\delta)$ is increasing with respect to $t$ and for any $\chi=\left(t, w, z,\left\{u_{i}\right\}_{i \in \mathbb{N}}, v\right) \in \mathbb{S}$,

$$
|\nu(\chi)| \leq \delta\|\chi\|_{\theta}+K_{t}(\delta),
$$

where $\|\chi\|_{\theta}^{2}:=t^{2}+|w|^{2}+|z|^{2}+\sum_{i \in \mathbb{N}} \theta_{i}\left|u_{i}\right|^{2}+|v|^{2}$. 
(ii) $\nu$ is Hölder continuous on $\mathbb{S}$, that is, there exist $\beta \in(0,1]$ and $\|\nu\|_{\beta}>0$ such that for any $\chi=\left(t, w, z,\left\{u_{i}\right\}_{i \in \mathbb{N}}, v\right), \chi^{\prime}=\left(t^{\prime}, w^{\prime}, z^{\prime},\left\{u_{i}^{\prime}\right\}_{i \in \mathbb{N}}, v^{\prime}\right) \in \mathbb{S}$,

$$
\left|\nu(\chi)-\nu\left(\chi^{\prime}\right)\right| \leq\|\nu\|_{\beta}\left(\left|t-t^{\prime}\right|^{\beta / 2}+\left|w-w^{\prime}\right|^{\beta}+\left|z-z^{\prime}\right|^{\beta}+\sum_{i \in \mathbb{N}} \theta_{i}\left|u_{i}-u_{i}^{\prime}\right|^{\beta}+\left|v-v^{\prime}\right|^{\beta}\right) .
$$

(iii) The functions $\zeta$ and $c$ are $\gamma$-Hölder continuous in space and $\gamma / 2$-Hölder continuous in time with $\gamma \in(0,1]$, that is,

$$
\begin{aligned}
\|\zeta\|_{\gamma} & :=\sup _{t \in[0, \infty), x \neq y} \frac{|\zeta(t, x)-\zeta(t, y)|}{|x-y|^{\gamma}}+\sup _{x \in \mathbb{R}^{d}, t \neq s} \frac{|\zeta(t, x)-\zeta(s, x)|}{|t-s|^{\gamma / 2}}<\infty, \\
\|c\|_{\gamma} & :=\sup _{t \in[0, \infty), x \neq y} \frac{|c(t, x)-c(t, y)|}{|x-y|^{\gamma}}+\sup _{x \in \mathbb{R}^{d}, t \neq s} \frac{|c(t, x)-c(s, x)|}{|t-s|^{\gamma / 2}}<\infty .
\end{aligned}
$$

(iv) $\sigma$ is uniformly elliptic matrix, that is, there exists a such that for any $\xi \in \mathbb{R}^{d}$,

$$
\underline{a}|\xi|^{2} \leq\left\langle\sigma \sigma^{\top} \xi, \xi\right\rangle \text {. }
$$

Under Assumption 3.15 the drift coefficients $\nu \circ A$. and $\nu \circ A^{(n, m)}$ satisfy Assumption 2.1 and 3.2. Indeed, since $\zeta$ and $c$ are of linear growth, there exists $C>0$ such that $\left\|A_{t}(w)\right\|_{\theta} \vee$ $\left\|A_{t}^{(n, m)}(w)\right\|_{\theta} \leq C\left(1+\left(1+\|\theta\|_{\ell_{1}}\right) w_{t}^{*}\right)$. Therefore, from Theorem 3.1 there exist pdfs $p_{t}(x, \cdot)$ and $p_{t}^{(n, m)}(x, \cdot)$ of $X_{t}^{x}$ and $X_{t}^{(x, n, m)}$, respectively such that for all $y \in \mathbb{R}^{d}$,

$$
\begin{aligned}
p_{t}(x, y) & =g_{t \sigma}(x, y)+\int_{0}^{t} \mathbb{E}\left[\left\langle\nabla_{x} g_{(t-s) \sigma}\left(X_{s}^{x}, y\right), \nu\left(A_{s}\left(X^{x}\right)\right)\right\rangle\right] \mathrm{d} s \\
p_{t}^{(n, m)}(x, y) & =g_{t \sigma}(x, y)+\int_{0}^{t} \mathbb{E}\left[\left\langle\nabla_{x} g_{(t-s) \sigma}\left(X_{s}^{(x, n, m)}, y\right), \nu\left(A_{s}^{(n, m)}\left(X^{(x, n, m)}\right)\right)\right\rangle\right] \mathrm{d} s .
\end{aligned}
$$

Under Assumption 3.15, we have the following error estimate.

Lemma 3.16. Suppose Assumption 3.15 holds. Then for any $p>1$, there exists $C_{p}>0$ such that

$$
\sup _{0 \leq s \leq t} \mathbb{E}\left[\left|\nu\left(A_{s}(x+\sigma W)\right)-\nu\left(A_{s}^{(n, m)}(x+\sigma W)\right)\right|^{p}\right]^{1 / p} \leq C_{p}\left\{\left(\frac{\log n}{n}\right)^{\beta \gamma / 2}+\sum_{i=m+1}^{\infty} \theta_{i}\right\} .
$$

Proof. It suffices to show the statement for $p>1 / \beta$. Since $\nu$ is $\beta$-Hölder continuous, we have for any $s \in[0, t]$,

$$
\begin{aligned}
& \frac{\left|\nu\left(A_{s}(x+\sigma W)\right)-\nu\left(A_{s}^{(n, m)}(x+\sigma W)\right)\right|^{p}}{6^{p-1}\|\nu\|_{\beta}^{p}} \\
& \leq\left|s-\eta_{n}(s)\right|^{p \beta}+|\sigma|^{p \beta}\left|W_{s}-W_{\eta_{n}(s)}\right|^{p \beta}+\left|\max _{0 \leq u \leq s} \zeta\left(u, x+\sigma W_{u}\right)-\max _{0 \leq u \leq s} \zeta\left(\eta_{n}(u), x+\sigma W_{\eta_{n}(u)}\right)\right|^{p \beta}
\end{aligned}
$$




$$
\begin{aligned}
& +|\sigma|^{p \beta}\left(\sum_{i=1}^{m} \theta_{i}\left|W_{\tau_{i}(s)}-W_{\eta_{n}\left(\tau_{i}(s)\right)}\right|^{\beta}+\sum_{i=m+1}^{\infty} \theta_{i}\left|x+W_{\tau_{i}(s)}\right|^{\beta}\right)^{p}+\left|\int_{\eta_{n}(s)}^{s} c\left(u, x+\sigma W_{u}\right) \mathrm{d} u\right|^{p \beta} \\
& +t^{p \beta-1} \int_{0}^{\eta_{n}(s)}\left|c\left(u, x+\sigma W_{u}\right)-c\left(\eta_{n}(u), x+\sigma W_{\eta_{n}(u)}\right)\right|^{p \beta} \mathrm{d} u \\
& \leq \frac{T^{p \beta / 2}}{n^{p \beta / 2}}+|\sigma|^{p \beta}\left|W_{s}-W_{\eta_{n}(s)}\right|^{p \beta}+2^{p \beta-1}\|\zeta\|_{\gamma}^{p \beta}\left\{\frac{T^{p \beta \gamma / 2}}{n^{p \beta \gamma / 2}}+|\sigma|^{p \beta \gamma} \max _{0 \leq u \leq s}\left|W_{u}-W_{\eta_{n}(u)}\right|^{p \beta \gamma}\right\} \\
& +|\sigma|^{p \beta}\left(\|\theta\|_{\ell_{1}} \max _{0 \leq u \leq s}\left|W_{u}-W_{\eta_{n}(u)}\right|^{\beta}+\max _{0 \leq u \leq s}\left|x+W_{u}\right|^{\beta} \sum_{i=m+1}^{\infty} \theta_{i}\right)^{p}+\frac{T^{p \beta}}{n^{p \gamma}} \sup _{0 \leq s \leq T}\left|c\left(s, x+\sigma W_{s}\right)\right|^{p \beta} \\
& +t^{p \gamma}\|c\|_{\gamma}^{p \beta \gamma}\left\{\frac{T^{p \beta \gamma / 2}}{n^{p \beta \gamma / 2}}+|\sigma|^{p \beta \gamma} \int_{0}^{T}\left|W_{u}-W_{\eta_{n}(u)}\right|^{p \beta \gamma} \mathrm{d} u\right\} .
\end{aligned}
$$

By using modulus continuity of Brownian motion (see, Theorem 2.9.25 in [28]), there exists $C_{p}>0$ such that

$$
\sup _{0 \leq s \leq t} \mathbb{E}\left[\left|\nu\left(A_{s}(x+\sigma W)\right)-\nu\left(A_{s}^{(n, m)}(x+\sigma W)\right)\right|^{p}\right]^{1 / p} \leq C_{p}\left\{\left(\frac{\log n}{n}\right)^{\beta \gamma / 2}+\sum_{i=m+1}^{\infty} \theta_{i}\right\},
$$

which concludes the proof.

As an conclusion of the comparison Theorem 3.14 and Lemma 3.16, we obtain the following error estimate.

Corollary 3.17. Suppose Assumption 3.15 holds. Assume that $f: \mathbb{R}^{d} \rightarrow \mathbb{R}$ is a measurable function satisfies the exponentially bounded, that is, there exist $K>0$ such that for all $x \in \mathbb{R}^{d}$, $|f(x)| \leq K \exp (K|x|)$. Then for any $p_{1}, p_{2}, p_{3}>1$ with $1 / p_{1}+1 / p_{2}+1 / p_{3}=1$ and $p_{1} \in(1, d /(d-$ $1)$ ), there exist $C_{+}>0$ and $c_{+}>0$ such that for any $(t, x, y) \in[0, T] \times \mathbb{R}^{d} \times \mathbb{R}^{d}$, if $t \in\left(0, t_{2 p_{2}}\right]$, then

$$
\begin{aligned}
\left|p_{t}(x, y)-p_{t}^{(n, m)}(x, y)\right| & \leq C_{+} \exp \left(c_{+}\left(1+|x|^{2}\right) t\right)(1+|x|)^{2} g_{p_{1} c_{+} t}(x, y)\left\{\left(\frac{\log n}{n}\right)^{\beta \gamma / 2}+\sum_{i=m+1}^{\infty} \theta_{i}\right\}, \\
\left|\mathbb{E}\left[f\left(X_{T}^{x}\right)\right]-\mathbb{E}\left[f\left(X_{T}^{(x, n, m)}\right)\right]\right| & \leq C_{+} \exp \left(c_{+}\left(1+|x|^{2}\right) t\right)(1+|x|)^{2}\left\{\left(\frac{\log n}{n}\right)^{\beta \gamma / 2}+\sum_{i=m+1}^{\infty} \theta_{i}\right\},
\end{aligned}
$$

and if $t \in\left(t_{2 p_{2}}, T\right]$,

$$
\begin{aligned}
\left|p_{t}(x, y)-p_{t}^{(n, m)}(x, y)\right| & \leq C_{+} \exp \left(\frac{|x|^{2}}{16 \widehat{c}_{+} p_{2} T}\right)(1+|x|)^{2} g_{p_{1} \widehat{c} t}(x, y)\left\{\left(\frac{\log n}{n}\right)^{\beta \gamma / 2}+\sum_{i=m+1}^{\infty} \theta_{i}\right\}, \\
\left|\mathbb{E}\left[f\left(X_{T}^{x}\right)\right]-\mathbb{E}\left[f\left(X_{T}^{(x, n, m)}\right)\right]\right| & \leq C_{+} \exp \left(\frac{|x|^{2}}{16 \widehat{c}_{+} p_{2} T}\right)(1+|x|)^{2}\left\{\left(\frac{\log n}{n}\right)^{\beta \gamma / 2}+\sum_{i=m+1}^{\infty} \theta_{i}\right\},
\end{aligned}
$$

where $t_{2 p_{2}}$ is defined by (18). 
Remark 3.18. The main idea of the proof for Corollary 3.17 (and Theorem 3.14) is to use Maruyama-Girsanov transform. This idea is inspired by Mackevičius 41] who study the weak rate of convergence of the Euler-Maruyama scheme for the SDE $\mathrm{d} X_{t}=b\left(X_{t}\right) \mathrm{d} t+\sigma \mathrm{d} W_{t}$ under Lipschitz condition on $b$. However, we would like to point out that the proof in 41] contains several gaps (see, for instance Lemma 2 in [41], see also Remark 3.3. in [50]).

\subsection{Markovian SDEs with unbounded drift}

In this section, we consider the parametrix expansion similar to (32) on a pdf of a solution of the following Markov type SDE of the form

$$
X_{t}^{x}=x+\int_{0}^{t} b\left(X_{s}^{x}\right) \mathrm{d} s+\int_{0}^{t} \sigma\left(X_{s}^{x}\right) \mathrm{d} W_{s}, t \geq 0, x \in \mathbb{R}^{d} .
$$

Let us consider a frozen process $X^{s, x, z}=\left(X_{t}^{s, x, z}\right)_{t \in[s, T]}$ for $x, z \in \mathbb{R}^{d}$ defined by

$$
\widetilde{X}_{t}^{s, x, z}:=x+\sigma(z)\left(W_{t}-W_{s}\right) .
$$

We denote $p^{z}(s, x ; t, \cdot)$ a pdf of $\widetilde{X}_{t}^{s, x, z}$ for $t \in(0, T]$ and $x, z \in \mathbb{R}^{d}$.

If the drift coefficients $b$ is bounded measurable, and the diffusion coefficient $\sigma$ satisfies Assumption 2.1(ii) and (iii), then by the same way as section 4.1 in [39] and approximation arguments (see Remark 4.1 in [39]), it holds that

$$
p_{t}(x, y)=\sum_{n=0}^{\infty} \bar{p} \otimes H^{\otimes n}(0, x ; t, y),
$$

where $\bar{p}(s, x ; t, y):=p^{y}(s, x ; t, y)$ and

$$
H(s, x ; t, y):=\left\langle\nabla_{x} \bar{p}(s, x ; t, y), b(x)\right\rangle+\sum_{i, j=1}^{d} \frac{a_{i, j}(x)-a_{i, j}(y)}{2} \partial_{x_{i} x_{j}}^{2} \bar{p}(s, x ; t, y) .
$$

Remark 3.19. In [39], the authors define a frozen process by $\widetilde{X}_{t}^{s, x, z}:=x+b(z)(t-s)+\sigma(z)\left(W_{t}-\right.$ $W_{s}$ ). However, even if one define (45), the parametrix expansion (46) can be shown by the same way as in 39.

Assumption 3.20. (i) the drift coefficient $b$ is of linear growth, that is, there exists $K>0$ such that for any $x \in \mathbb{R}^{d}$,

$$
|b(x)| \leq K(1+|x|) .
$$

(ii) $b$ is of sub-linear growth, that is, for any $\delta>0$, there exists $K(\delta)>0$ such that for all $x \in \mathbb{R}^{d}$

$$
|b(x)| \leq \delta|x|+K(\delta) .
$$

Under the above conditions, the parametrix expansion (46) still holds. 
Theorem 3.21. Suppose that Assumption 2.1] (ii), (iii) and Assumption [3.20 hold. Then the series $\sum_{n=0}^{\infty} \bar{p} \otimes H^{\otimes n}(0, x ; t, y)$ converges absolutely and uniformly for $(t, y) \in(0, T] \times \mathbb{R}^{d}$, and a pdf of $X_{t}^{x}$ denoted by $p_{t}(x, y)$ satisfies the expansion

$$
p_{t}(x, y)=\sum_{n=0}^{\infty} \bar{p} \otimes H^{\otimes n}(0, x ; t, y) .
$$

In order to provide the parametrix expansion for a pdf of a solution of SDE (44), under unbounded drift coefficients we first show that if $b$ satisfies Assumption 2.1(ii), (iii) and Assumption 3.20. the expansion of right hand side in (46) convergences absolutely and uniformly in $x, y \in$ $\mathbb{R}^{d}$. Then by taking bounded measurable approximation of $b$, we show the convergences of the parametrix expansion (47) by using comparison property (see, Theorem 3.14). below.

We denote $|H|(s, z ; t, y):=|H(s, z ; t, y)|$, and the following classical estimate will be used

Lemma 3.22. Let $A$ be a $d \times d$ matrix and suppose that there exists $\underline{A}$ and $\bar{A}$ such that for all $\xi \in \mathbb{R}^{d}, \underline{A}|\xi|^{2} \leq\langle a \xi, \xi\rangle \leq \bar{A}|\xi|^{2}$. Then for $\alpha \in(0,1]$, there exists $C$ such that for all $(t, x, y) \in$ $(0, T] \times \mathbb{R}^{d} \times \mathbb{R}^{d}$,

$$
|y-x|^{\alpha}\left|H_{t A}^{i, j}(y-x)\right| g_{t A}(x, y) \leq \frac{C}{t^{\left(1-\frac{\alpha}{2}\right)}} g_{2 t \bar{A}}(x, y),
$$

where $H_{A}^{i, j}(y):=\left(A^{-1} y\right)^{i}\left(A^{-1} y\right)^{j}-\left(A^{-1}\right)_{i, j}$.

The proof of Lemma 3.22 follows by using the classical estimation $\sup _{x \in \mathbb{R}^{d}}|x|^{q} e^{-|x|}<\infty$ for any $q \geq 0$.

Lemma 3.23. Suppose that Assumption 2.1 (ii), (iii) and Assumption 3.20 (i) hold. Then there exists $C_{+}$and $c_{+}$such that for any $n \in \mathbb{N} \cup\{0\}$ and $(t, x, y) \in(0, T] \times \mathbb{R}^{d} \times \mathbb{R}^{d}$,

$$
\begin{aligned}
\bar{p} \otimes|H|^{\otimes n}(0, x ; t, y) & \leq \frac{\left(C_{+}(1+|x|) t^{\alpha / 2} \Gamma(\alpha / 2)\right)^{n}}{\Gamma(1+n \alpha / 2)} g_{\widehat{c}_{+} t}(x, y), \\
\sum_{n=0}^{\infty} \bar{p} \otimes|H|^{\otimes n}(0, x ; t, y) & <\infty .
\end{aligned}
$$

Proof. We first show (48). For $n=0$, it is obvious from the definition of $\bar{p}$ and Assumption 2.1 (ii). For $n=1$, we have

$$
\begin{aligned}
\bar{p} \otimes|H|(0, x ; t, y) & =\int_{0}^{t} \int_{\mathbb{R}^{d}} \bar{p}\left(0, x ; t_{1}, y_{1}\right)\left|H\left(t_{1}, y_{1} ; t, y\right)\right| \mathrm{d} y_{1} \mathrm{~d} t_{1} \\
& \leq \bar{p} \otimes\left|H^{b}\right|(0, x ; t, y)+\bar{p} \otimes\left|H^{a}\right|(0, x ; t, y),
\end{aligned}
$$

where $\left|H^{b}\right|$ and $\left|H^{a}\right|$ are defined by

$$
\left|H^{b}\right|(s, z ; t, y)=\left|\left\langle\nabla_{x} \bar{p}(s, z ; t, y), b(z)\right\rangle\right|,\left|H^{a}\right|(s, z ; t, y)=\left|\sum_{i, j=1}^{d} \frac{a_{i, j}(z)-a_{i, j}(y)}{2} \partial_{z_{i} z_{j}}^{2} \bar{p}(s, z ; t, y)\right| .
$$


From the Gaussian upper bound (8) for $\bar{p}$, we obtain

$$
\begin{aligned}
\bar{p} \otimes\left|H^{b}\right|(0, x ; t, y) & \leq \widehat{C}_{+} \int_{0}^{t} \int_{\mathbb{R}^{d}} g_{\widehat{c}_{+} s}(x, z)\left|\left\langle\nabla_{x} \bar{p}(s, z ; t, y), b(s, z)\right\rangle\right| \mathrm{d} z \mathrm{~d} s \\
& =\widehat{C}_{+} \int_{0}^{t} \mathbb{E}\left[\left|\left\langle\nabla_{x} \bar{p}\left(s, \sqrt{\widehat{c}_{+}} W_{s}+x ; t, y\right), b\left(s, \sqrt{\widehat{c}_{+}} W_{s}+x\right)\right\rangle\right|\right] \mathrm{d} s .
\end{aligned}
$$

It follows from Hölder's inequality, the Gaussian upper bound (9) for $\nabla_{x} \bar{p}$, Lemma 2.4 and analogy of Lemma 3.8 (ii) that for $p_{1}, p_{2}>1$ with $p_{1} \in\left(1, \frac{d}{d-1}\right)$ and $1 / p_{1}+1 / p_{2}=1$,

$$
\begin{aligned}
\bar{p} \otimes\left|H^{b}\right|(0, x ; t, y) & \leq \widehat{C}_{+} \int_{0}^{t} \mathbb{E}\left[\left|\nabla_{x} \bar{p}\left(s, \sqrt{\widehat{c}_{+}} W_{s}+x ; t, y\right)\right|\left|b\left(t_{1}, \sqrt{\widehat{c}_{+}} W_{s}+x\right)\right|\right] \mathrm{d} s \\
& \leq \widehat{C}_{+} \int_{0}^{t} \mathbb{E}\left[\left|\nabla_{x} \bar{p}\left(s, \sqrt{\widehat{c}_{+}} W_{s}+x ; t, y\right)\right|^{p_{1}}\right]^{1 / p_{1}} \mathbb{E}\left[\left|b\left(s, \sqrt{\widehat{c}_{+}} W_{s}+x\right)\right|^{p_{2}}\right]^{1 / p_{2}} \mathrm{~d} s \\
& \leq C(1+|x|) \int_{0}^{t} \frac{1}{(t-s)^{1 / 2}} \mathbb{E}\left[\left|g_{\widehat{c}_{+}(t-s)}\left(\sqrt{\widehat{c}_{+}} W_{s}+x, y\right)\right|^{p_{1}}\right]^{1 / p_{1}} \mathrm{~d} s \\
& \leq C^{\prime}(1+|x|) g_{\widehat{c}_{+}(t-s)}(x, y) \int_{0}^{t} \frac{1}{(t-s)^{1 / 2+d\left(p_{1}-1\right) / 2 p_{1}}} \mathrm{~d} s,
\end{aligned}
$$

for some $C, C^{\prime}>0$. By using Assumption 2.1 (ii), Lemma 3.22 and Chapman-Kolmogorov equation, we have

$$
\begin{aligned}
\bar{p} \otimes\left|H^{a}\right|(0, x ; t, y) & \leq \frac{\|a\|_{\alpha}}{2} \int_{0}^{t} \int_{\mathbb{R}^{d}} \bar{p}(0, x ; s, z)|y-z|^{\alpha}\left|\partial_{z_{i} z_{j}}^{2} \bar{p}(s, x ; t, y)\right| \mathrm{d} z \mathrm{~d} s \\
& =\frac{\|a\|_{\alpha}}{2} \int_{0}^{t} \int_{\mathbb{R}^{d}} \bar{p}(0, x ; s, z)|y-z|^{\alpha}\left|H_{(t-s) a(y)}^{i, j}(y-z)\right| \bar{p}(s, z ; t, y) \mathrm{d} z \mathrm{~d} s \\
& \leq C \int_{0}^{t} \int_{\mathbb{R}^{d}} \frac{1}{(t-s)^{1-\alpha / 2}} g_{\widehat{c}_{+} s}(x, z) g_{2 \widehat{c}_{+}(t-s)}(z, y) \mathrm{d} z \mathrm{~d} s \\
& \leq C^{\prime} g_{c_{+} t}(x, y) \int_{0}^{t} \frac{1}{(t-s)^{1-\alpha / 2}} \mathrm{~d} s
\end{aligned}
$$

for some $C, C^{\prime}>0$ and $c_{+}>0$. By choosing $p_{1}=\frac{d}{d-(1-\alpha)}$, (48) holds for $n=1$. We assume that (48) holds for $n-1$. Then we have

$$
\begin{aligned}
\bar{p} \otimes|H|^{\otimes n}(0, x ; t, y) & =\int_{0}^{t} \int_{\mathbb{R}^{d}} \bar{p} \otimes|H|^{\otimes n-1}(0, x ; s, z)|H(s, z ; t, y)| \mathrm{d} z \mathrm{~d} s \\
& \leq \frac{\left(C_{+}(1+|x|) \Gamma(\alpha / 2)\right)^{n-1}}{\Gamma(1+(n-1) \alpha / 2)} \int_{0}^{t} \int_{\mathbb{R}^{d}} s^{(n-1) \alpha / 2} g_{c_{+} s}(x, z)|H(s, z ; t, y)| \mathrm{d} z \mathrm{~d} s .
\end{aligned}
$$

Hence from the same arguments for $n=1$, it holds that

$$
\begin{aligned}
\bar{p} \otimes|H|^{\otimes n}(0, x ; t, y) & \leq \frac{\left(C_{+}(1+|x|)\right)^{n} \Gamma(\alpha / 2)^{n-1}}{\Gamma(1+(n-1) \alpha / 2)} g_{\widehat{c}_{+} t}(x, y) \int_{0}^{t} \frac{s^{(n-1) \alpha / 2}}{(t-s)^{1-\alpha / 2}} \mathrm{~d} s \\
& =\frac{\left(C_{+}(1+|x|) t^{\alpha / 2} \Gamma(\alpha / 2)\right)^{n}}{\Gamma(1+n \alpha / 2)} g_{\widehat{c}_{+} t}(x, y) .
\end{aligned}
$$


Hence (48) holds for every $n \in \mathbb{N} \cup\{0\}$. By using (48), we obtain (49).

Now we define an approximation $b_{N}$ of $b$ by

$$
b_{N}(x)= \begin{cases}b(x) & \text { if }|x| \leq N \\ b(N x /|x|) & \text { if }|x|>N\end{cases}
$$

Then from Assumption 2.1 (ii), (iii) and Assumption 3.20, $b_{N}$ satisfies the following conditions

(i) $b_{N}$ is bounded, that is, $\left|b_{N}(x)\right| \leq K(1+N)$ for any $x \in \mathbb{R}^{d}$.

(ii) For any $x \in \mathbb{R}^{d}, b_{N}(x) \rightarrow b(x)$ as $N \rightarrow+\infty$.

(iii) $b_{N}$ is of linear growth uniformly in $N \in \mathbb{N}$, that is, there exists $K>0$ such that for any $x \in \mathbb{R}^{d}$,

$$
\sup _{N \in \mathbb{N}}\left|b_{N}(x)\right| \leq K(1+|x|) .
$$

(iv) $b_{N}$ is of sub-linear growth uniformly in $N \in \mathbb{N}$, that is, for any $\delta>0$, there exists $K(\delta)>0$ such that for any $x \in \mathbb{R}^{d}$

$$
\sup _{N \in \mathbb{N}}\left|b_{N}(x)\right| \leq \delta|x|+K(\delta) .
$$

Proof of Theorem 3.21. We consider the following SDE with drift coefficient $b_{N}$

$$
X_{t}^{N, x}=x+\int_{0}^{t} b_{N}\left(X_{s}^{N, x}\right) \mathrm{d} s+\int_{0}^{t} \sigma\left(X_{s}^{N, x}\right) \mathrm{d} W_{s}, t \geq 0, x \in \mathbb{R}^{d} .
$$

Then $X_{t}^{N, x}$ admits a pdf, denoted by $p_{t}^{N}(x, \cdot)$, and from (46) the following parametrix expansion holds

$$
p_{t}^{N}(x, y)=\sum_{n=0}^{\infty} \bar{p} \otimes H_{N}^{\otimes n}(s, x ; t, y)
$$

where $H_{N}$ is defined by

$$
H_{N}(s, x ; t, y):=\left\langle\nabla_{x} \bar{p}(s, x ; t, y), b_{N}(x)\right\rangle+\sum_{i, j=1}^{d} \frac{a_{i, j}(x)-a_{i, j}(y)}{2} \partial_{x_{i} x_{j}}^{2} \bar{p}(s, x ; t, y) .
$$

Moreover, since $b_{N}$ is of linear growth uniformly in $N \in \mathbb{N}$, and from Lemma 3.23, we have the following estimation uniformly in $N \in \mathbb{N}$

$$
\sum_{n=0}^{\infty} \bar{p} \otimes\left|\sup _{N \in \mathbb{N}} H_{N}\right|^{\otimes n}(s, x ; t, y)<\infty,
$$

for all $0 \leq s<t \leq T$ and $x, y \in \mathbb{R}^{d}$. By using comparison Theorem 3.14 and Lemma 3.23, (49) and dominated convergence theorem, we have

$$
p_{t}(x, y)=\lim _{N \rightarrow+\infty} p_{t}^{N}(x, y)=\lim _{N \rightarrow+\infty} \sum_{n=0}^{\infty} \bar{p} \otimes H_{N}^{\otimes n}(s, x ; t, y)=\sum_{n=0}^{\infty} \bar{p} \otimes H^{\otimes n}(s, x ; t, y) .
$$

for all $x \in \mathbb{R}$ and a.e. $y \in \mathbb{R}^{d}$. Hence we conclude the statement (47). 


\subsection{Application to unbiased simulation scheme}

In this subsection, we introduce a probabilistic representation of a pdf of a solution of Markovian SDE (44), in order to provide an unbiased simulation scheme.

We introduce the define of counting process.

Definition 3.24. Let $R_{t}:=\sum_{n=1}^{\infty} \mathbf{1}\left(\tau_{n} \leq t\right)$ where $\left(\tau_{n}-\tau_{n-1}\right)_{n \in \mathbb{N}}$ with $\tau_{0}=0$ are independent and identically distributed random variables with $p d f \zeta$. Then we call $R=\left(R_{t}\right)_{t \geq 0}$ the counting process with $\pi:=\left(\tau_{n}\right)_{n \in \mathbb{N}}$ and $\zeta$.

Example 3.1. Let $\zeta(t):=\lambda e^{-\lambda t} \mathbf{1}_{[0, \infty)}(t)$. Then $R=\left(R_{t}\right)_{t \geq 0}$ is a Poisson process with parameter $\lambda>0$. Another choice of $\zeta$ is $\zeta(t):=\frac{A}{t^{\beta}} \mathbf{1}_{[0,2 T]}(t)$ where $A:=(1-\beta) /(2 T)^{1-\beta}$ and $\beta \in(0,1)$. For more on this, see [1].

The following lemma plays a crucial role in our argument.

Lemma 3.25 (Lemma 7.3 in [31]). Let $R=\left(R_{t}\right)_{t \geq 0}$ be a counting process with jumps times $\pi:=\left(\tau_{n}\right)_{n \in \mathbb{N}}$ and $\zeta$. Then for any $t>0, n \in \mathbb{N}$ and any bounded measurable function $V_{n}: \mathbb{R}^{n} \rightarrow \mathbb{R}$,

$$
\begin{aligned}
& \mathbb{E}\left[\mathbf{1}_{\left\{R_{t}=n\right\}} V_{n}\left(\tau_{1}, \ldots, \tau_{n}\right)\right] \\
& =\int_{0}^{t} \mathrm{~d} s_{n} \int_{0}^{s_{n}} \mathrm{~d} s_{n-1} \cdots \int_{0}^{s_{2}} \mathrm{~d} s_{1} V_{n}\left(s_{1}, \ldots, s_{n}\right)\left(1-F_{\zeta}\left(t-s_{n}\right)\right) \prod_{i=0}^{n-1} \zeta\left(s_{i+1}-s_{i}\right),
\end{aligned}
$$

where $F_{\zeta}(x):=\int_{-\infty}^{x} \zeta(y) d y$ and $s_{0}=0$.

We define $\phi_{t}^{y}(x):=\bar{p}(0, x ; t, y)$ and

$$
\widehat{\theta}_{t}(x, y):=-\sum_{i=1}^{d} b_{i}(x) H_{t a(y)}^{i}(y-x)+\sum_{i, j=1}^{d} \frac{a_{i, j}(x)-a_{i, j}(y)}{2} H_{t a(y)}^{i, j}(y-x) .
$$

Then it holds that $H(s, x ; t, y)=\widehat{\theta}_{t-s}(x, y) \phi_{t-s}^{y}(x)$. By using this fact, we obtain the following representation for a pdf, which provide an unbiased simulation scheme for an expectation $\mathbb{E}\left[f\left(X_{t}^{x}\right)\right]$.

Corollary 3.26. Suppose that Assumption 2.1 (ii), (iii) and Assumption 3.20 hold. Let $R=$ $\left(R_{t}\right)_{t \geq 0}$ be a counting process with $\pi:=\left(\tau_{n}\right)_{n \in \mathbb{N}}$ and $\zeta$, which is independent from $W$. For any $t \in(0, T]$, a pdf $p_{t}(x, \cdot)$ of $X_{t}^{x}$ satisfies the following probabilistic representation

$$
p_{t}(x, y)=\mathbb{E}\left[\frac{\bar{p}\left(t-\tau_{t}, x, X_{\tau_{t}}^{*, \pi}(y)\right)}{1-F_{\zeta}\left(t-\tau_{t}\right)} \Gamma_{t}(y)\right]
$$

where $\tau_{t}:=\tau_{R_{t}}, F_{\zeta}(t):=\int_{0}^{t} \zeta(s) \mathrm{d} s$ and

$$
\Gamma_{t}(y):=\mathbf{1}_{\left\{R_{t}=0\right\}}+\prod_{j=0}^{R_{t}-1} \frac{\widehat{\theta}_{\tau_{j+1}-\tau_{j}}\left(X_{\tau_{j+1}^{*}}^{*, \pi}(y), X_{\tau_{j}}^{*, \pi}(y)\right)}{\zeta\left(\tau_{j+1}-\tau_{j}\right)} \mathbf{1}_{\left\{R_{t} \geq 1\right\}},
$$


and $X^{*, \pi}(y)$ is the Euler-Maruyama scheme with $X_{0}^{*, \pi}(y)=y$ and a random partition $\pi$ which drift coefficient is zero and diffusion coefficient is $\sigma$, that is, $X_{0}^{*, \pi}(y):=y$ and for $j \geq 1$,

$$
X_{\tau_{j}}^{*, \pi}(y):=X_{\tau_{j-1}}^{*, \pi}(y)+\sigma\left(X_{\tau_{j-1}}^{*, \pi}(y)\right)\left(W_{\tau_{j}}-W_{\tau_{j-1}}\right) .
$$

Moreover, for a random variable $Z$ with a pdf $g$, which is independent from $R$ and $W$, and for any measurable function $f: \mathbb{R}^{d} \rightarrow \mathbb{R}$ with $\mathbb{E}\left[\left|f\left(X_{t}^{x}\right)\right|\right]<\infty$, it holds that

$$
\mathbb{E}\left[f\left(X_{t}^{x}\right)\right]=\mathbb{E}\left[\frac{f(Z)}{g(Z)} \frac{\bar{p}\left(t-\tau_{t}, x, X_{\tau_{t}}^{*, \pi}(Z)\right)}{1-F_{\zeta}\left(t-\tau_{t}\right)} \Gamma_{t}(Z)\right] .
$$

Since we have the expansion (47) for unbounded drift coefficient $b$, we can prove Corollary 3.26 by a similar argument to the proof of Theorem 5.7, Proposition 7.2 in [4, Theorem 7.4 in 31] and Theorem 3.2 in 34. For the convenience of the reader, we will give a proof below.

Proof of Corollary 3.26. It follows from (49) that the series $\sum_{n=0}^{\infty} \bar{p} \otimes H^{\otimes n}(0, x ; t, y)$ converges absolutely and uniformly for $(t, y) \in(0, T] \times \mathbb{R}^{d}$, thus using Fubini's theorem and the equation $H(s, x ; t, y)=\widehat{\theta}_{t-s}(x, y) \phi_{t-s}^{y}(x)$, we have for each $n \in \mathbb{N} \cup\{0\}$,

$$
\begin{aligned}
& \bar{p} \otimes H^{\otimes n}(0, x ; t, y)=\int_{0}^{t_{0}} \mathrm{~d} t_{1} \cdots \int_{0}^{t_{n-1}} \mathrm{~d} t_{n} \int_{\mathbb{R}^{n}} \mathrm{~d} y_{1} \cdots \mathrm{d} y_{n} \prod_{i=0}^{n-1} \widehat{\theta}_{t_{i}-t_{i+1}}\left(y_{i+1}, y_{i}\right) \phi_{t_{i}-t_{i+1}}^{y_{i}}\left(y_{i+1}\right) \phi_{t_{n}}^{y_{n}}(x), \\
& =\int_{0}^{t} \mathrm{~d} s_{n} \int_{0}^{s_{n}} \mathrm{~d} s_{n-1} \cdots \int_{0}^{s_{2}} \mathrm{~d} s_{1} \int_{\mathbb{R}^{n}} \mathrm{~d} y_{1} \cdots \mathrm{d} y_{n} \prod_{i=0}^{n-1} \widehat{\theta}_{s_{i+1}-s_{i}}\left(y_{i+1}, y_{i}\right) \phi_{s_{i+1}-t_{i}}^{y_{i}}\left(y_{i+1}\right) \phi_{t-s_{n}}^{y_{n}}(x),
\end{aligned}
$$

where $\left(t_{0}, y_{n+1}\right):=(t, y)$ and in the last equality we use the change of variables $s_{n}=t_{0}-t_{n}$. For any partition $\pi_{0}=\left(s_{i}\right)_{i \in \mathbb{N}}$ with $0=: s_{0} \leq s_{1}<\cdots<s_{n}<\cdots<\infty$, we define a Markov chain $X^{*, \pi_{0}}(y)$ as follows: $X_{0}^{*, s_{0}}(y):=y$ and $\mathbb{P}\left(X_{s_{i}}^{*, \pi_{0}}(y) \in \mathrm{d} y_{i+1} \mid X_{s_{i-1}}^{*, \pi_{0}}(y)=y_{i}\right)=\varphi_{s_{i}-s_{i-1}}^{y_{i}}\left(y_{i+1}\right) \mathrm{d} y_{i+1}$. Then, by using the Markov property of stochastic process $y+\sigma(y) W_{t}$ whose density is $\phi_{t}^{y}$, the parametrix expansion (47) and Lemma 3.25 we conclude the statement.

\subsection{Hölder continuity of pdf}

In this subsection, by using a regularity of $q(s, x ; t, y)$, we prove that if the diffusion matrix is smooth, then a pdf $p_{t}(x, \cdot)$ of a solution of SDE (1) is Hölder continuous.

Theorem 3.27. Suppose Assumption 2.1 and 3.2 hold, and $\sigma(t, \cdot) \in C_{b}^{2}\left(\mathbb{R}^{d} ; \mathbb{R}^{d \times d}\right)$ for all $t \in[0, T]$. Let $p_{t}(x, \cdot)$ be a continuous version of a pdf of a solution $X_{t}^{x}$ to $S D E$ (1), for $t \in(0, T]$ and $x \in \mathbb{R}^{d}$. Assume $\gamma \in(0,1)$ and $p_{1}, p_{2}, p_{3}>1$ with $p_{1} \in\left(1, \frac{d}{d-(1-\gamma)}\right), 1 / p_{1}+1 / p_{2}+1 / p_{3}=1$.

(i) There exists $C_{\gamma, p_{1}}>0$ and $c_{p_{1}}>0$ such that, for all $(t, x, y) \in(0, T] \times \mathbb{R}^{d} \times \mathbb{R}^{d}$

$$
\begin{aligned}
& \left|p_{t}(x, y)-p_{t}\left(x, y^{\prime}\right)\right| \\
& \leq C_{\gamma, p_{1}} \sup _{0 \leq s \leq t} \mathbb{E}\left[Z_{t}\left(1, Y^{0, x}\right)^{p_{2}}\right]^{1 / p_{2}} \mathbb{E}\left[\left|b\left(s, Y^{0, x}\right)\right|^{p_{3}}\right]^{1 / p_{3}} \frac{\left|y-y^{\prime}\right|^{\gamma}}{t^{\gamma / 2}}\left\{g_{c_{p_{1}} t}(x, y)+g_{c_{p_{1}} t}\left(x, y^{\prime}\right)\right\} .
\end{aligned}
$$


(ii) Recall that $t_{r}$ is defined in (18). There exists $C_{\gamma, p_{1}}>0$ and $c_{p_{1}}>0$ such that, for all $(t, x, y) \in(0, T] \times \mathbb{R}^{d} \times \mathbb{R}^{d}$, it holds that if $t \in\left(0, t_{p_{1}}\right]$,

$$
\begin{aligned}
& \left|p_{t}(x, y)-p_{t}\left(x, y^{\prime}\right)\right| \\
& \leq C_{\gamma, p_{1}}(1+|x|) \exp \left(c_{p_{1}}\left(1+|x|^{2}\right) t\right) \frac{\left|y-y^{\prime}\right|^{\gamma}}{t^{\gamma / 2}}\left\{g_{c_{p_{1}} t}(x, y)+g_{c_{p_{1}} t}\left(x, y^{\prime}\right)\right\},
\end{aligned}
$$

and if $t \in\left(t_{p_{1}}, T\right]$, then

$$
\begin{aligned}
& \left|p_{t}(x, y)-p_{t}\left(x, y^{\prime}\right)\right| \\
& \leq C_{\gamma, p_{1}}(1+|x|) \exp \left(\frac{|x|^{2}}{8 p_{2} \widehat{c}_{+} T}\right) \frac{\left|y-y^{\prime}\right|^{\gamma}}{t^{\gamma / 2}}\left\{g_{c_{p_{1}} t}(x, y)+g_{c_{p_{1}} t}\left(x, y^{\prime}\right)\right\} .
\end{aligned}
$$

Remark 3.28. Theorem 3.27 implies that the regularity of a pdf of a solution to SDE (1D) does not depend on the regularity of drift coefficient.

From Theorem 3.4 (i), the representation (11) is continuous. In order to prove Theorem 3.27 we need to consider the Hölder continuity of the pdf $q(0, x, t ; \cdot)$ and its derivative.

Lemma 3.29. Suppose Assumption 2.1 and 3.2 hold, and $\sigma(t, \cdot) \in C_{b}^{2}\left(\mathbb{R}^{d} ; \mathbb{R}^{d \times d}\right)$ for all $t \in[0, T]$. Then there exist $\widetilde{C}_{+}>0$ and $\widetilde{c}_{+}>\widehat{c}_{+}>0$ such that for any $\gamma \in(0,1), y, y^{\prime} \in \mathbb{R}^{d}, 0 \leq s<t \leq T$ and $i=1, \ldots, d$,

$$
\begin{aligned}
\left|q(s, x ; t, y)-q\left(s, x ; t, y^{\prime}\right)\right| & \leq \frac{\widetilde{C}_{+}\left|y-y^{\prime}\right|^{\gamma}}{t^{\gamma / 2}}\left\{g_{\widetilde{c}_{+}(t-s)}(x, y)+g_{\widetilde{c}_{+}(t-s)}\left(x, y^{\prime}\right)\right\}, \\
\left|\partial_{x_{i}} q(s, x ; t, y)-\partial_{x_{i}} q\left(s, x ; t, y^{\prime}\right)\right| & \leq \frac{\widetilde{C}_{+}\left|y-y^{\prime}\right|^{\gamma}}{(t-s)^{1-(1-\gamma) / 2}}\left\{g_{\widetilde{c}_{+}(t-s)}(x, y)+g_{\widetilde{c}_{+}(t-s)}\left(x, y^{\prime}\right)\right\} .
\end{aligned}
$$

Proof. We only prove (51). The proof of (50) is similar. Note that if $\sigma(t, \cdot) \in C_{b}^{2}$, then it holds that there exist $\widehat{C}_{+}>0$ and $\widehat{c}_{+}>0$ such that for all $t \in(0, T], x, y \in \mathbb{R}^{d}$ and $i, j=1, \ldots, d$,

$$
\left|\partial_{x_{i}} q(s, x ; t, y)\right| \leq \frac{\widehat{C}_{+}}{(t-s)^{1 / 2}} g_{\widehat{c}_{+}(t-s)}(x, y) \text { and }\left|\partial_{x_{i}} \partial_{y_{j}} q(s, x ; t, y)\right| \leq \frac{\widehat{C}_{+}}{t-s} g_{\widehat{c}_{+}(t-s)}(x, y),
$$

(see, Chapter 9, section 6, Theorem 7 in [15]).

We first assume $\left|y-y^{\prime}\right|^{2}<t-s$. Then by the mean-value theorem and (52), we have

$$
\begin{aligned}
\left|\partial_{x_{i}} q(s, x ; t, y)-\partial_{x_{i}} q\left(s, x ; t, y^{\prime}\right)\right| & =\left|\int_{0}^{1}\left\langle y-y^{\prime}, \nabla_{y} \partial_{x_{i}} q(s, x ; t, \zeta(\theta))\right\rangle \mathrm{d} \theta\right| \\
& \leq \frac{\sqrt{d} \widehat{C}_{+}\left|y-y^{\prime}\right|}{t} \int_{0}^{1} g_{\widehat{c}_{+}(t-s)}(x, \zeta(\theta)) \mathrm{d} \theta,
\end{aligned}
$$

where $\zeta(\theta)=\theta y+(1-\theta) y^{\prime}$. Since $\left|y-y^{\prime}\right|^{2}<t-s$, by using $\left|x_{1}-x_{2}\right|^{2} \geq \frac{1}{2}\left|x_{1}\right|^{2}-\left|x_{2}\right|^{2}$ for any $x_{1}, x_{2} \in \mathbb{R}^{d}$, we have

$-\frac{|\zeta(\theta)-x|^{2}}{\widehat{c}_{+}(t-s)} \leq-\frac{|y-x|^{2}}{2 \widehat{c}_{+}(t-s)}+\frac{(1-\theta)\left|y-y^{\prime}\right|^{2}}{\widehat{c}_{+}(t-s)} \leq-\frac{|y-x|^{2}}{2 \widehat{c}_{+}(t-s)}+\frac{1}{\widehat{c}_{+}}, \quad-\frac{|\zeta(\theta)-x|^{2}}{\widehat{c}_{+}(t-s)} \leq-\frac{\left|y^{\prime}-x\right|^{2}}{2 \widehat{c}_{+}(t-s)}+\frac{1}{\widehat{c}_{+}}$. 
Hence we have

$$
\begin{aligned}
& \left|\partial_{x_{i}} q(s, x ; t, y)-\partial_{x_{i}} q\left(s, x ; t, y^{\prime}\right)\right| \\
& \leq \frac{C^{\prime}\left|y-y^{\prime}\right|^{\gamma}}{(t-s)^{1-(1-\gamma) / 2}} \frac{\left(|y-x|^{1-\gamma}+\left|y^{\prime}-x\right|^{1-\gamma}\right)}{(t-s)^{(1-\gamma) / 2}} \exp \left(-\frac{|y-x|^{2}}{16 \widehat{c}_{+}(t-s)}\right) \exp \left(-\frac{\left|y^{\prime}-x\right|^{2}}{16 \widehat{c}_{+}(t-s)}\right) \\
& \quad \times\left\{g_{4 \widehat{c}_{+}(t-s)}(x, y)+g_{4 \widehat{c}_{+}(t-s)}\left(x, y^{\prime}\right)\right\} \\
& \leq \frac{C^{\prime \prime}\left|y-y^{\prime}\right|^{\gamma}}{(t-s)^{1-(1-\gamma) / 2}}\left\{g_{4 \widehat{c}_{+}(t-s)}(x, y)+g_{4 \widehat{c}_{+}(t-s)}\left(x, y^{\prime}\right)\right\},
\end{aligned}
$$

for some $C^{\prime}, C^{\prime \prime}$. This concludes (51) for $\left|y-y^{\prime}\right|^{2}<t-s$.

If $\left|y-y^{\prime}\right|^{2} \geq t$, by using (52), we have

$$
\begin{aligned}
& \left|\partial_{x_{i}} q(s, x ; t, y)-\partial_{x_{i}} q\left(s, x ; t, y^{\prime}\right)\right| \leq\left|\partial_{x_{i}} q(s, x ; t, y)\right|+\left|\partial_{x_{i}} q\left(s, x ; t, y^{\prime}\right)\right| \\
& \leq \frac{2 \widehat{C}_{+}(t-s)^{\gamma / 2}}{(t-s)^{1-(1-\gamma) / 2}}\left\{g_{\widehat{c}_{+}(t-s)}(x, y)+g_{\widehat{c}_{+}(t-s)}\left(x, y^{\prime}\right)\right\} \leq \frac{2 \widehat{C}_{+}\left|y-y^{\prime}\right|^{\gamma}}{(t-s)^{1-(1-\gamma) / 2}}\left\{g_{\widehat{c}_{+}(t-s)}(x, y)+g_{\widehat{c}_{+}(t-s)}\left(x, y^{\prime}\right)\right\},
\end{aligned}
$$

which concludes (51) for $\left|y-y^{\prime}\right|^{2} \geq t-s$.

Proof of Theorem 3.27. From Theorem 3.1 and Lemma 3.29, it is sufficient to estimate

$$
\left|\int_{0}^{t} \mathbb{E}\left[\left\langle\nabla_{x} q\left(x, X_{s}^{x} ; t, y\right)-\nabla_{x} q\left(s, X_{s}^{x} ; t, y^{\prime}\right), b\left(s, X^{x}\right)\right\rangle\right] \mathrm{d} s\right| .
$$

By using Theorem [2.3. Hölder's inequality, Jensen's inequality, Lemma 2.4. Lemma 3.29 and (8), for any $p_{1}, p_{2}, p_{3}>1$ with $1 / p_{1}+1 / p_{2}+1 / p_{3}=1$, (53) is bounded by

$$
\begin{aligned}
& \int_{0}^{t} \mathbb{E}\left[\left|\nabla_{x} q\left(s, Y_{s}^{0, x} ; t, y\right)-\nabla_{x} q\left(s, Y_{s}^{0, x} ; t, y^{\prime}\right)\right|^{p_{1}}\right]^{1 / p_{1}} \mathbb{E}\left[Z_{s}\left(1, Y^{0, x}\right)^{p_{2}}\right]^{1 / p_{2}} \mathbb{E}\left[\left|b\left(s, Y^{0, x}\right)\right|^{p_{3}}\right]^{1 / p_{3}} \mathrm{~d} s \\
& \leq \sup _{0 \leq s \leq t} \mathbb{E}\left[Z_{t}\left(1, Y^{0, x}\right)^{p_{2}}\right]^{1 / p_{2}} \mathbb{E}\left[\left|b\left(s, Y^{0, x}\right)\right|^{p_{3}}\right]^{1 / p_{3}} \\
& \quad \times \int_{0}^{t}\left(\int_{\mathbb{R}^{d}}\left|\nabla_{x} q(s, z ; t, y)-\nabla_{x} q\left(s, z ; t, y^{\prime}\right)\right|^{p_{1}} q(0, x ; s, z) \mathrm{d} z\right)^{1 / p_{1}} \mathrm{~d} s \\
& \leq C \sup _{0 \leq s \leq t} \mathbb{E}\left[Z_{t}\left(1, Y^{0, x}\right)^{p_{2}}\right]^{1 / p_{2}} \mathbb{E}\left[\left|b\left(s, Y^{0, x}\right)\right|^{p_{3}}\right]^{1 / p_{3}} \\
& \quad \times \int_{0}^{t}\left(\int_{\mathbb{R}^{d}} \frac{\left|y-y^{\prime}\right|^{\gamma p_{1}}\left\{g_{\widetilde{c}_{+}(t-s) / p_{1}}(z, y)+g_{\widetilde{c}_{+}(t-s) / p_{1}}\left(z, y^{\prime}\right)\right\}}{(t-s)^{p_{1}(1-(1-\gamma) / 2)+\left(p_{1}-1\right) d / 2}} g_{\widetilde{c}_{+} s}(x, z) \mathrm{d} z\right)^{1 / p_{1}} \mathrm{~d} s,
\end{aligned}
$$

for some $C>0$. Since $1 / p_{1}<1, g_{c(t-s) / p_{1}}(z, y) \leq C_{p_{1}} g_{c(t-s)}(z, y)$ for some $C_{p_{1}}>0$ and since $\gamma \in(0,1)$, it holds that

$$
\rho:=(1-(1-\gamma) / 2)+\frac{\left(p_{1}-1\right) d}{2 p_{1}}<1 \quad \Leftrightarrow \quad 1<p_{1}<\frac{d}{d-(1-\gamma)} .
$$


Therefore, by using Chapman-Kolmogorov equation, (54) is estimated by

$$
\begin{aligned}
& C^{\prime} \sup _{0 \leq s \leq t} \mathbb{E}\left[Z_{t}\left(1, Y^{0, x}\right)^{p_{2}}\right]^{1 / p_{2}} \mathbb{E}\left[\left|b\left(s, Y^{0, x}\right)\right|^{p_{3}}\right]^{1 / p_{3}} \frac{C_{p_{1}}^{1 / p_{1}} t^{1-\rho}}{1-\rho}\left|y-y^{\prime}\right|^{\gamma}\left\{g_{\widetilde{c}_{+} t}(x, y)+g_{\widetilde{c}_{+} t}\left(x, y^{\prime}\right)\right\}^{1 / p_{1}} \\
& \leq C^{\prime \prime} \sup _{0 \leq s \leq t} \mathbb{E}\left[Z_{t}\left(1, Y^{0, x}\right)^{p_{2}}\right]^{1 / p_{2}} \mathbb{E}\left[\left|b\left(s, Y^{0, x}\right)\right|^{p_{3}}\right]^{1 / p_{3}} \frac{t^{1-\rho+\frac{d}{2}\left(1-\frac{1}{p_{1}}\right)}}{1-\rho}\left|y-y^{\prime}\right|^{\gamma}\left\{g_{\widetilde{c}_{+} p_{1} t}(x, y)+g_{\widetilde{c}_{+} p_{1} t}\left(x, y^{\prime}\right)\right\},
\end{aligned}
$$

for some $C^{\prime}, C^{\prime \prime}>0$. Since $t^{1-\rho+\frac{d}{2}\left(1-\frac{1}{p_{1}}\right)}=t^{(1-\gamma) / 2} \leq T t^{-\gamma / 2}$, we conclude the statement (i). Lemma 2.4 and Lemma 3.6 with $r=p_{2}$ imply the statement (ii).

\section{One-dimensional SDEs with super-linear growth coeffi- cients}

In this section, inspired by [25, 58, 59], we use a "one-step" tamed Euler-Maruyama approximation, in order to prove the existence of a pdf for a solution of one-dimensional Markovian SDEs

$$
X_{t}=x+\int_{0}^{t} b\left(s, X_{s}\right) \mathrm{d} s+\int_{0}^{t} \sigma\left(X_{s}\right) \mathrm{d} W_{s},(t, x) \in[0, T] \times \mathbb{R},
$$

under the assumption that the coefficients $b:[0, T] \times \mathbb{R} \rightarrow \mathbb{R}$ and $\sigma: \mathbb{R} \rightarrow \mathbb{R}$ satisfies the following conditions.

Assumption 4.1. We suppose that the coefficients $b:[0, T] \times \mathbb{R} \rightarrow \mathbb{R}$ and $\sigma: \mathbb{R} \rightarrow \mathbb{R}$ are measurable and satisfy the following conditions:

(i) (Khasminskii and one-sided Lipschitz condition) There exist $K>0, p_{0}>2$ and $p_{1}>2$ such that for each $x, y \in \mathbb{R}$ and $s \in[0, T]$,

$$
\begin{aligned}
2 x b(s, x)+\left(p_{0}-1\right)|\sigma(x)|^{2} & \leq K\left(1+|x|^{2}\right), \\
2(x-y)(b(s, x)-b(s, y))+\left(p_{1}-1\right)|\sigma(x)-\sigma(y)|^{2} & \leq K|x-y|^{2} .
\end{aligned}
$$

(ii) (locally Lipschitz continuous) There exist $K>0$ and $\ell \in\left(0, \frac{p_{0}-2}{4}\right]$ such that for each $x, y \in \mathbb{R}$ and $s \in[0, T]$,

$$
\begin{aligned}
|b(s, x)-b(s, y)| & \leq K\left(1+|x|^{\ell}+|y|^{\ell}\right)|x-y|, \\
|b(s, x)| & \leq K\left(1+|x|^{\ell+1}\right) .
\end{aligned}
$$

Example 4.1. Let $b(s, x) \equiv b(x):=\lambda x(\mu-|x|)$ and $\sigma(x):=\xi|x|^{3 / 2}$ for some $\lambda, \mu, \xi>0$, then the SDE (55) is called Heston-3/2 volatility model (see, e.g. [18]) and for any $p_{0} \leq \frac{2 \lambda+|\xi|^{2}}{|\xi|^{2}}$ and $p_{1} \leq \frac{\lambda+|\xi|^{2}}{|\xi|^{2}}$, and $x, y \in \mathbb{R}$, it holds that

$$
\begin{aligned}
2 x b(x)+\left(p_{0}-1\right)|\sigma(x)|^{2} & \leq 2 \lambda \mu\left(1+|x|^{2}\right), \\
2(x-y)(b(x)-b(y))+\left(p_{1}-1\right)|\sigma(x)-\sigma(y)|^{2} & \leq 2 \lambda \mu|x-y|^{2}, \\
|b(x)-b(y)| & \leq \lambda(\mu \wedge 1)(1+|x|+|y|)|x-y|,
\end{aligned}
$$

(see, Appendix in [59]). 
Remark 4.2. (i) From Assumption 4.1, we have there exist $K_{0}, K_{1}, K_{2}>0$ such that for any $x, y \in \mathbb{R}$ and $s \in[0, T]$,

$$
\begin{aligned}
\left(p_{0}-1\right)|\sigma(x)|^{2} & \leq K\left(1+|x|^{2}\right)-2 x b(s, x) \leq K+2 K|x|+K|x|^{2}+2 K|x|^{\ell+2} \\
& \leq\left(p_{0}-1\right) K_{0}\left(1+|x|^{\ell+2}\right),
\end{aligned}
$$

and

$$
\begin{aligned}
\left(p_{1}-1\right)|\sigma(x)-\sigma(y)|^{2} & \leq K|x-y|^{2}-2(x-y)(b(s, x)-b(s, y)) \\
& \leq K\left(3+2|x|^{\ell}+2|y|^{\ell}\right)|x-y|^{2} \\
& \leq\left(p_{1}-1\right) K_{1}\left(1+|x|^{\ell}+|y|^{\ell}\right)|x-y|^{2} \\
& \leq\left(p_{1}-1\right) K_{2}\left(1+|x|^{2 \ell}+|y|^{2 \ell}\right)|x-y|^{2} .
\end{aligned}
$$

(ii) If the coefficients $b$ and $\sigma$ are local Lipschitz continuous in space, then there exists a unique strong solution to the equation (55]), (e.g., Theorem 5.2.5 in [28]).

Under the above assumptions, we prove the existence of a pdf.

Theorem 4.3. Suppose that Assumption 4.1 holds. Then for any $t \in(0, T], X_{t}$ admits a pdf on the set $\{x \in \mathbb{R} ; \sigma(x) \neq 0\}$.

We derive a several lemmas for proving the above theorem. The following lemma shows that the Assumption 4.1 (ii) implies that the solution of SDE (55) has a moment and satisfies a Kolmogorov type condition.

Lemma 4.4. Suppose that Assumption 4.1 holds. Then, for any $p \in\left[2, p_{0}\right]$, there exists $C_{p}>0$ such that

$$
\sup _{t \in[0, T]} \mathbb{E}\left[\left|X_{t}\right|^{p}\right] \leq C_{p} .
$$

Moreover, there exists $C>0$ such that for any $t, s \in[0, T]$ with $s<t$, we have

$$
\mathbb{E}\left[\left|X_{t}-X_{s}\right|^{2}\right] \leq C(t-s) .
$$

Proof. It suffices to prove the statement for $p=p_{0}$. Let $t \in[0, T]$ and $\tau_{N}:=\inf \left\{t \geq 0 ;\left|X_{t}\right| \geq N\right\}$ for $N \in \mathbb{N}$. By using Itô's formula for $|x|^{p_{0}}$ and Assumption 4.1] (i), we have

$$
\begin{aligned}
\left|X_{t \wedge \tau_{N}}\right|^{p_{0}} & =|x|^{p_{0}}+\int_{0}^{t \wedge \tau_{N}}\left\{p_{0} X_{s}^{p_{0}-1} b\left(s, X_{s}\right)+\frac{p_{0}\left(p_{0}-1\right)}{2} X_{s}^{p_{0}-2} \sigma\left(X_{s}\right)^{2}\right\} \mathrm{d} s+\int_{0}^{t \wedge \tau_{N}} p_{0} X_{s}^{p_{0}-1} \sigma\left(X_{s}\right) \mathrm{d} W_{s} \\
& \leq|x|^{p_{0}}+\frac{p_{0}}{2} \int_{0}^{t \wedge \tau_{N}} X_{s}^{p_{0}-2}\left\{2 X_{s} b\left(s, X_{s}\right)+\left(p_{0}-1\right) \sigma\left(X_{s}\right)^{2}\right\} \mathrm{d} s+\int_{0}^{t \wedge \tau_{N}} p_{0} X_{s}^{p_{0}-1} \sigma\left(X_{s}\right) \mathrm{d} W_{s} \\
& \leq|x|^{p_{0}}+\frac{p_{0} K}{2} \int_{0}^{t \wedge \tau_{N}} X_{s}^{p_{0}-2}\left(1+X_{s}^{2}\right) \mathrm{d} s+\int_{0}^{t \wedge \tau_{N}} p_{0} X_{s}^{p_{0}-1} \sigma\left(X_{s}\right) \mathrm{d} W_{s} .
\end{aligned}
$$

By taking expectation, there exists $C>0$ such that we have

$$
\mathbb{E}\left[\left|X_{t \wedge \tau_{N} \mid}\right|^{p_{0}}\right] \leq C+C \int_{0}^{t} \mathbb{E}\left[\left|X_{s \wedge \tau_{N}}\right|^{p_{0}}\right] \mathrm{d} s,
$$


and thus Gronwall's inequality implies

$$
\mathbb{E}\left[\left|X_{t \wedge \tau_{N}}\right|^{p_{0}}\right] \leq C e^{C T} .
$$

By letting $N \rightarrow \infty$, we concludes (58).

From Assumption 4.1 (ii), (56) and (58), we have

$$
\int_{s}^{t} \mathbb{E}\left[\left|b\left(u, X_{u}\right)\right|^{2}\right] \mathrm{d} u+\int_{s}^{t} \mathbb{E}\left[\left|\sigma\left(X_{u}\right)\right|^{2}\right] \mathrm{d} u \leq C(t-s),
$$

for some $C>0$. Therefore, by using Jensen's inequality and Itô's isometry, we conclude (59)).

Now inspired by [14] and [25, 58, 59], we consider a one-step tamed Euler-Maruyama scheme. For a fixed $t \in(0, T]$ and $\varepsilon \in(0,1)$, we define a one-step tamed Euler-Maruyama scheme $X^{(\varepsilon)}=$ $\left(X_{u}^{(\varepsilon)}\right)_{u \in[0, t]}$ by

$$
\begin{aligned}
X_{u}^{(\varepsilon)} & := \begin{cases}X_{u}, & \text { if } u \in[0, t-\varepsilon], \\
X_{t-\varepsilon}+\int_{t-\varepsilon}^{u} b_{\varepsilon}\left(s, X_{t-\varepsilon}\right) \mathrm{d} s+\int_{t-\varepsilon}^{u} \sigma_{\varepsilon}\left(X_{t-\varepsilon}\right) \mathrm{d} W_{s}, & \text { if } u \in(t-\varepsilon, t]\end{cases} \\
& =x+\int_{0}^{u} \bar{b}_{s}^{(\varepsilon)} \mathrm{d} s+\int_{0}^{u} \bar{\sigma}_{s}^{(\varepsilon)} \mathrm{d} W_{s},
\end{aligned}
$$

where

$$
b_{\varepsilon}(s, x):=\frac{b(s, x)}{1+\varepsilon^{1 / 2}|x|^{\ell}}, \quad \sigma_{\varepsilon}(x):=\frac{\sigma(x)}{1+\varepsilon^{1 / 2}|x|^{\ell / 2}},
$$

and

$$
\bar{b}_{s}^{(\varepsilon)}:=\left\{\begin{array}{lll}
b\left(s, X_{s}\right) & \text { if } s \in[0, t-\varepsilon], \\
b_{\varepsilon}\left(s, X_{t-\varepsilon}\right) & \text { if } s \in(t-\varepsilon, t],
\end{array} \bar{\sigma}_{s}^{(\varepsilon)}:= \begin{cases}\sigma\left(X_{s}\right) & \text { if } s \in[0, t-\varepsilon], \\
\sigma_{\varepsilon}\left(X_{t-\varepsilon}\right) & \text { if } s \in(t-\varepsilon, t] .\end{cases}\right.
$$

Then it holds that for any $x \in \mathbb{R}, s \in[0, T]$ and $\varepsilon \in(0,1)$,

$$
\begin{aligned}
\left|b_{\varepsilon}(s, x)\right| & \leq\left\{K \varepsilon^{-1 / 2}(1+|x|)\right\} \wedge|b(s, x)|, \\
\left|\sigma_{\varepsilon}(x)\right|^{2} & \leq\left\{K_{0} \varepsilon^{-1}\left(1+|x|^{2}\right)\right\} \wedge|\sigma(x)|^{2},
\end{aligned}
$$

and

$$
\begin{aligned}
\left|b(s, x)-b_{\varepsilon}(s, x)\right| & =\frac{|b(s, x)||x|^{\ell} \varepsilon^{1 / 2}}{1+\varepsilon^{1 / 2}|x|^{\ell}} \leq K\left(1+|x|^{\ell+1}\right)|x|^{\ell} \varepsilon^{1 / 2}, \\
\left|\sigma(x)-\sigma_{\varepsilon}(x)\right|^{2} & =\frac{|\sigma(x)|^{2}|x|^{\ell} \varepsilon}{\left(1+\varepsilon^{1 / 2}|x|^{\ell / 2}\right)^{2}} \leq K_{0}\left(1+|x|^{\ell+2}\right)|x|^{\ell} \varepsilon .
\end{aligned}
$$

Indeed, it holds from Assumption 4.1 (ii) and the inequality $(1+|x|)\left(1+|x|^{\ell}\right) \geq 1+|x|^{\ell+1}$ that

$$
\left|b_{\varepsilon}(s, x)\right| \leq \frac{K \varepsilon^{-1 / 2}\left(1+|x|^{\ell+1}\right)}{1+|x|^{\ell}} \leq K \varepsilon^{-1 / 2}(1+|x|),
$$


which implies (60). By the similar way, , it holds from (56) and the inequality $\left(1+|x|^{l / 2}\right)^{2}\left(1+|x|^{2}\right) \geq$ $1+|x|^{l+2}$ that

$$
\left|\sigma_{\varepsilon}(s, x)\right|^{2} \leq \frac{K_{0} \varepsilon^{-1}\left(1+|x|^{l+2}\right)}{\left(1+|x|^{l / 2}\right)^{2}} \leq K_{0} \varepsilon^{-1}\left(1+|x|^{2}\right),
$$

which implies (61). (62) and (63) are followed from Assumption 4.1 (ii) and (566), respectively.

Remark 4.5. As mentioned in introduction, Romito [57] studied the existence and Besov regularity of the probability density function of a solution of SDEs with locally bounded drift, and locally Hölder continuous, elliptic diffusion coefficient. Therefore, the statement of Theorem 4.3 isincluded in their results (see, Theorem 4.1 in [57]). In [57], Romito use a localization argument and one-step Euler-Maruyama scheme. On the other hand, in our paper, we use directly one-step tamed Euler scheme, so the approach of proof is different.

By using Lemma 4.4, $X^{(\varepsilon)}$ has a moment and some error estimate.

Lemma 4.6. Suppose that Assumption 4.1 holds. Then, for any $p \in\left[2, p_{0}\right]$, there exists $C_{p}>0$ such that

$$
\sup _{\varepsilon \in(0,1)} \sup _{u \in[0, t]} \mathbb{E}\left[\left|X_{u}^{(\varepsilon)}\right|^{p}\right] \leq C_{p}
$$

Moreover, for any $p \in\left[1, \frac{2 p_{0}}{\ell+2}\right]$, there exists $C_{p}>0$ such that

$$
\sup _{u \in[t-\varepsilon, t]} \mathbb{E}\left[\left|X_{u}^{(\varepsilon)}-X_{t-\varepsilon}^{(\varepsilon)}\right|^{p}\right] \leq C_{p} \varepsilon^{p / 2} .
$$

Proof. If $u \in[0, t-\varepsilon]$, then $X_{u}^{(\varepsilon)}=X_{u}$ thus, we have the (64) from Lemma 4.4

Now we assume that $u \in[t-\varepsilon, t]$, then by using (60) and (61) with $\left|\sigma_{\varepsilon}(x)\right| \leq|\sigma(x)|$, since $\sigma\left(X_{t-\varepsilon}\right)$ and $W_{u}-W_{t-\varepsilon}$ are independent,

$$
\begin{aligned}
\mathbb{E}\left[\left|X_{u}^{(\varepsilon)}\right|^{p}\right] \leq & 3^{p-1} \mathbb{E}\left[\left|X_{t-\varepsilon}\right|^{p}+\varepsilon^{p-1} \int_{t-\varepsilon}^{t}\left|b_{\varepsilon}\left(s, X_{t-\varepsilon}\right)\right|^{p} \mathrm{~d} s+\left|\sigma_{\varepsilon}\left(X_{t-\varepsilon}\right)\right|^{p}\left|W_{u}-W_{t-\varepsilon}\right|^{p}\right] \\
\leq & 3^{p-1} \mathbb{E}\left[\left|X_{t-\varepsilon}\right|^{p}\right]+3^{p-1} K^{p} \varepsilon^{p / 2} \mathbb{E}\left[\left(1+\left|X_{t-\varepsilon}\right|\right)^{p}\right] \\
& +3^{p-1} K_{0}^{p / 2} \varepsilon^{-p / 2} \mathbb{E}\left[\left(1+\left|X_{t-\varepsilon}\right|^{2}\right)^{p / 2}\right] \mathbb{E}\left[\left|W_{u}-W_{t-\varepsilon}\right|^{p}\right] .
\end{aligned}
$$

Since $\mathbb{E}\left[\left|W_{u}-W_{t-\varepsilon}\right|^{p}\right] \leq C \varepsilon^{p / 2}$ for some $C>0$, thus we obtain (64).

Now we prove (65). By using (56), (60) and (61) since $\sigma\left(X_{t-\varepsilon}\right)$ and $W_{u}-W_{t-\varepsilon}$ are independent, we have

$$
\begin{aligned}
\mathbb{E}\left[\left|X_{u}^{(\varepsilon)}-X_{t-\varepsilon}^{(\varepsilon)}\right|^{p}\right] & \leq 2^{p-1} \varepsilon^{p-1} \int_{t-\varepsilon}^{t} \mathbb{E}\left[\left|b_{\varepsilon}\left(s, X_{t-\varepsilon}\right)\right|^{p}\right] \mathrm{d} s+2^{p-1} \mathbb{E}\left[\left|\sigma\left(X_{t-\varepsilon}\right)\right|^{p}\right] \mathbb{E}\left[\left|W_{u}-W_{t-\varepsilon}\right|^{p}\right] \\
& \leq 2^{p-1} K^{p} \varepsilon^{p / 2} \mathbb{E}\left[\left(1+\left|X_{t-\varepsilon}\right|\right)^{p}\right]+2^{3 p / 2-2} K_{0}^{p / 2} \mathbb{E}\left[1+\left|X_{t-\varepsilon}\right|^{\frac{p(\ell+2)}{2}}\right] \mathbb{E}\left[\left|W_{u}-W_{t-\varepsilon}\right|^{p}\right] .
\end{aligned}
$$

The assumption $p \in\left[1, \frac{2 p_{0}}{\ell+2}\right]$ implies $\frac{p(\ell+2)}{2} \leq p_{0}$, thus from Lemma 4.4 we conclude (65). 
Remark 4.7. Note that in the inequality (66), for the drift coefficient $b_{\varepsilon}$, if we use the estimate $\left|b_{\varepsilon}(x)\right| \leq|b(x)|$, we need a stronger assumption $p \in\left[1, \frac{p_{0}}{\ell+1}\right]$.

The following lemma plays a crucial role in our argument.

Lemma 4.8. Suppose that Assumption 4.1 holds. Then there exists $C>0$ such that

$$
\mathbb{E}\left[\left|X_{t}-X_{t}^{(\varepsilon)}\right|^{2}\right] \leq C \varepsilon^{2} .
$$

Proof. By Itô's formula, we have for each $u \in[0, t]$

$$
\begin{aligned}
& \left|X_{u}-X_{u}^{(\varepsilon)}\right|^{2} \\
& =\int_{0}^{u}\left\{2\left(X_{s}-X_{s}^{(\varepsilon)}\right)\left(b\left(s, X_{s}\right)-\bar{b}_{s}^{(\varepsilon)}\right)+\left|\sigma\left(X_{s}\right)-\bar{\sigma}_{s}^{(\varepsilon)}\right|^{2}\right\} \mathrm{d} s+\int_{0}^{u}\left(X_{s}-X_{s}^{(\varepsilon)}\right)\left(\sigma\left(X_{s}\right)-\bar{\sigma}_{s}^{(\varepsilon)}\right) \mathrm{d} W_{s} \\
& =: I_{u}^{(\varepsilon)}+M_{u}^{(\varepsilon)} .
\end{aligned}
$$

Let $\tau_{N, \varepsilon}:=\inf \left\{s \geq 0 ;\left|X_{s}\right| \geq N\right\} \wedge \inf \left\{s \geq 0 ;\left|X_{s}^{(\varepsilon)}\right| \geq N\right\}$ for $N \in \mathbb{N}$. If $u \in[0, t-\varepsilon]$, then $I_{u}^{(\varepsilon)}=$ 0 . For $u \in[t-\varepsilon, t]$, by using an elementally inequality $\left|a_{1}+a_{2}+a_{3}\right|^{2} \leq(1+\rho) a_{1}^{2}+2\left(1+\rho^{-1}\right)\left(a_{2}^{2}+a_{3}^{2}\right)$, for all $\rho>0, a_{1}, a_{2}, a_{3} \geq 0$, we have

$$
\begin{aligned}
I_{u}^{(\varepsilon)}= & \int_{t-\varepsilon}^{u}\left\{2\left(X_{s}-X_{s}^{(\varepsilon)}\right)\left(b\left(s, X_{s}\right)-b_{\varepsilon}\left(s, X_{t-\varepsilon}\right)\right)+\left|\sigma\left(X_{s}\right)-\sigma_{\varepsilon}\left(X_{t-\varepsilon}\right)\right|^{2}\right\} \mathrm{d} s \\
\leq & \int_{t-\varepsilon}^{u}\left\{2\left(X_{s}-X_{s}^{(\varepsilon)}\right)\left(b\left(s, X_{s}\right)-b\left(s, X_{s}^{(\varepsilon)}\right)\right)+(1+\rho)\left|\sigma\left(X_{s}\right)-\sigma\left(X_{s}^{(\varepsilon)}\right)\right|^{2}\right\} \mathrm{d} s \\
& +\int_{t-\varepsilon}^{u}\left\{2\left(X_{s}-X_{s}^{(\varepsilon)}\right)\left(b\left(s, X_{s}^{(\varepsilon)}\right)-b\left(s, X_{t-\varepsilon}^{(\varepsilon)}\right)+2\left(1+\rho^{-1}\right)\left|\sigma\left(X_{s}^{(\varepsilon)}\right)-\sigma\left(X_{t-\varepsilon}^{(\varepsilon)}\right)\right|^{2}\right\} \mathrm{d} s\right. \\
& +\int_{t-\varepsilon}^{u}\left\{2\left(X_{s}-X_{s}^{(\varepsilon)}\right)\left(b\left(s, X_{t-\varepsilon}^{(\varepsilon)}\right)-b_{\varepsilon}\left(s, X_{t-\varepsilon}^{(\varepsilon)}\right)\right)+2\left(1+\rho^{-1}\right)\left|\sigma\left(X_{t-\varepsilon}^{(\varepsilon)}\right)-\sigma_{\varepsilon}\left(X_{t-\varepsilon}^{(\varepsilon)}\right)\right|^{2}\right\} \mathrm{d} s .
\end{aligned}
$$

We define $\rho:=\left(p_{1}-2\right) / 2>0$, then since $p_{1}>2,1+\rho<p_{1}-1$. Therefore, from Assumption 4.1 (i) and Young's inequality, there exists $C>0$ such that

$$
I_{u}^{(\varepsilon)} \leq(K+2) \int_{0}^{u}\left|X_{s}-X_{s}^{(\varepsilon)}\right|^{2} \mathrm{~d} s+C J_{u}^{(\varepsilon)}+C K_{u}^{(\varepsilon)},
$$

where for $u \in[t-\varepsilon, t]$,

$$
\begin{aligned}
J_{u}^{(\varepsilon)} & :=\int_{t-\varepsilon}^{u}\left\{\left|b\left(s, X_{s}^{(\varepsilon)}\right)-b\left(s, X_{t-\varepsilon}^{(\varepsilon)}\right)\right|^{2}+\left|\sigma\left(X_{s}^{(\varepsilon)}\right)-\sigma\left(X_{t-\varepsilon}^{(\varepsilon)}\right)\right|^{2}\right\} \mathrm{d} s \\
K_{u}^{(\varepsilon)} & :=\int_{t-\varepsilon}^{u}\left\{\left|b\left(s, X_{t-\varepsilon}^{(\varepsilon)}\right)-b_{\varepsilon}\left(s, X_{t-\varepsilon}^{(\varepsilon)}\right)\right|^{2}+\left|\sigma\left(X_{t-\varepsilon}^{(\varepsilon)}\right)-\sigma_{\varepsilon}\left(X_{t-\varepsilon}^{(\varepsilon)}\right)\right|^{2}\right\} \mathrm{d} s .
\end{aligned}
$$

Therefore, we have for any $u \in[0, t]$

$$
\mathbb{E}\left[\left|X_{u \wedge \tau_{N, \varepsilon}}-X_{u \wedge \tau_{N, \varepsilon}}^{(\varepsilon)}\right|^{2}\right] \leq(K+2) \int_{0}^{u} \mathbb{E}\left[\left|X_{s \wedge \tau_{N, \varepsilon}}-X_{s \wedge \tau_{N, \varepsilon}}^{(\varepsilon)}\right|^{2}\right] \mathrm{d} s+C \mathbb{E}\left[J_{t}^{(\varepsilon)}+K_{t}^{(\varepsilon)}\right] .
$$


Hence, by Gronwall's inequality, and then by taking $N \rightarrow \infty$, we have

$$
\mathbb{E}\left[\left|X_{t}-X_{t}^{(\varepsilon)}\right|^{2}\right] \leq e^{(K+2) T} \mathbb{E}\left[J_{t}^{(\varepsilon)}+K_{t}^{(\varepsilon)}\right] .
$$

By Assumption 4.1 (ii), (57) and Hölder's inequality, we have for any $p, q>1$ with $1 / p+1 / q=1$,

$$
\begin{aligned}
\mathbb{E}\left[J_{t}^{(\varepsilon)}\right] & \leq\left(3 K^{2}+K_{2}\right) \int_{t-\varepsilon}^{t} \mathbb{E}\left[\left(1+\left|X_{s}^{(\varepsilon)}\right|^{2 \ell}+\left|X_{t-\varepsilon}^{(\varepsilon)}\right|^{2 \ell}\right)\left|X_{s}^{(\varepsilon)}-X_{t-\varepsilon}^{(\varepsilon)}\right|^{2}\right] \mathrm{d} s \\
& \leq\left(3 K^{2}+K_{2}\right) \int_{t-\varepsilon}^{t} \mathbb{E}\left[\left(1+\left|X_{s}^{(\varepsilon)}\right|^{2 \ell}+\left|X_{t-\varepsilon}^{(\varepsilon)}\right|^{2 \ell}\right)^{p}\right]^{1 / p} \mathbb{E}\left[\left|X_{s}^{(\varepsilon)}-X_{t-\varepsilon}^{(\varepsilon)}\right|^{2 q}\right]^{1 / q} \mathrm{~d} s .
\end{aligned}
$$

Since $\ell \leq \frac{p_{0}-2}{4}$, by choosing $p=\frac{4 \ell+2}{3 \ell}$, then $q=\frac{p}{p-1}=\frac{4 \ell+2}{\ell+2}, 2 \ell p \leq \frac{2 p_{0}}{3}<p_{0}$ and $2 q \leq \frac{2 p_{0}}{\ell+2}$. Therefore, from Lemma 4.6, there exists $C>0$ such that

$$
\mathbb{E}\left[J_{t}^{(\varepsilon)}\right] \leq C \varepsilon^{2} .
$$

By Assumption 4.1 (ii), (62) and (63), we have

$$
\begin{aligned}
\mathbb{E}\left[K_{t}^{(\varepsilon)}\right] & \leq \varepsilon \int_{t-\varepsilon}^{t} \mathbb{E}\left[K^{2}\left(1+\left|X_{t-\varepsilon}^{(\varepsilon)}\right|^{\ell+1}\right)^{2}\left|X_{t-\varepsilon}^{(\varepsilon)}\right|^{2 \ell}+K_{0}\left(1+\left|X_{t-\varepsilon}^{(\varepsilon)}\right|^{\ell+2}\right)\left|X_{t-\varepsilon}^{(\varepsilon)}\right|^{\ell}\right] \mathrm{d} s \\
& =\varepsilon^{2} \mathbb{E}\left[K^{2}\left(1+\left|X_{t-\varepsilon}^{(\varepsilon)}\right|^{\ell+1}\right)^{2}\left|X_{t-\varepsilon}^{(\varepsilon)}\right|^{2 \ell}+K_{0}\left(1+\left|X_{t-\varepsilon}^{(\varepsilon)}\right|^{\ell+2}\right)\left|X_{t-\varepsilon}^{(\varepsilon)}\right|^{\ell}\right]
\end{aligned}
$$

Since $\ell \leq \frac{p_{0}-2}{4}, 2 \ell+2<4 \ell+2 \leq p_{0}$. Therefore, from Lemma 4.6, there exists $C>0$ such that

$$
\mathbb{E}\left[K_{t}^{(\varepsilon)}\right] \leq C \varepsilon^{2} .
$$

This concludes the statement.

Proof of Theorem 4.3. The proof is based on Theorem 2.1 in [14]. Let $\delta>0$. We define a function $f_{\delta}:[0, \infty) \rightarrow[0,1]$ such that

$$
f_{\delta}=0, \text { on }[0, \delta], \text { and } \sup _{x \neq y} \frac{\left|f_{\delta}(x)-f_{\delta}(y)\right|}{|x-y|} \leq 1 .
$$

We denote

$$
\mu_{X_{t}}(\mathrm{~d} x):=\mathbb{P}\left(X_{t} \in \mathrm{d} x\right), \mu_{\delta, X_{t}}(\mathrm{~d} x):=f_{\delta}(|\sigma(x)|) \mu_{X_{t}}(\mathrm{~d} x), \widehat{\mu}_{\delta, X_{t}}(\xi):=\int_{\mathbb{R}} e^{\sqrt{-1} \xi x} \mu_{\delta, X_{t}}(\mathrm{~d} x),
$$

for $t \in(0, T]$ and $\xi \in \mathbb{R}$. Then it follows from Lemma 1.1 and Lemma 1.2 in [14 that it is suffices to prove that $\widehat{\mu}_{\delta, X_{t}}$ satisfies

$$
\int_{\mathbb{R}}\left|\widehat{\mu}_{\delta, X_{t}}(\xi)\right|^{2} \mathrm{~d} \xi<\infty
$$

For any $\xi \in \mathbb{R}$, it holds that

$$
\left|\widehat{\mu}_{\delta, X_{t}}(\xi)\right|=\left|\int_{\mathbb{R}} e^{\sqrt{-1} \xi x} f_{\delta}(|\sigma(x)|) \mu_{X_{t}}(\mathrm{~d} x)\right|=\left|\mathbb{E}\left[e^{\sqrt{-1} \xi X_{t}} f_{\delta}\left(\left|\sigma\left(X_{t}\right)\right|\right)\right]\right|
$$




$$
\leq\left|\mathbb{E}\left[e^{\sqrt{-1} \xi X_{t}} f_{\delta}\left(\left|\sigma_{\varepsilon}\left(X_{t-\varepsilon}\right)\right|\right)\right]\right|+\mathbb{E}\left[\left|f_{\delta}\left(\left|\sigma\left(X_{t}\right)\right|\right)-f_{\delta}\left(\left|\sigma_{\varepsilon}\left(X_{t-\varepsilon}\right)\right|\right)\right|\right]
$$

Since $X_{t}=X_{t}^{(\varepsilon)}+\left(X_{t}-X_{t}^{(\varepsilon)}\right)$, by using the inequality $\left|e^{\sqrt{-1} \xi x}-e^{\sqrt{-1} \xi y}\right| \leq|\xi||x-y|,\left\|f_{\delta}\right\|_{\infty} \leq 1$ and Lipschitz continuity of $f_{\delta}$, we have

$$
\begin{aligned}
\left|\widehat{\mu}_{\delta, X_{t}}(\xi)\right| \leq & \left|\mathbb{E}\left[e^{\sqrt{-1} \xi X_{t}^{(\varepsilon)}} f_{\delta}\left(\left|\sigma_{\varepsilon}\left(X_{t-\varepsilon}\right)\right|\right)\right]\right|+|\xi| \mathbb{E}\left[\left|X_{t}-X_{t}^{(\varepsilon)}\right|\right] \\
& +\mathbb{E}\left[\left|\sigma\left(X_{t}\right)-\sigma\left(X_{t-\varepsilon}\right)\right|\right]+\mathbb{E}\left[\left|\sigma\left(X_{t-\varepsilon}\right)-\sigma_{\varepsilon}\left(X_{t-\varepsilon}\right)\right|\right]
\end{aligned}
$$

Note that

$$
\begin{aligned}
& \mathbb{E}\left[e^{\sqrt{-1} \xi X_{t}^{(\varepsilon)}} \mid \mathcal{F}_{t-\varepsilon}\right] \\
& =\exp \left(\sqrt{-1} \xi\left\{X_{t-\varepsilon}+\int_{t-\varepsilon}^{t} b_{\varepsilon}\left(s, X_{t-\varepsilon}\right) \mathrm{d} s\right\}\right) \mathbb{E}\left[\exp \left(\sqrt{-1} \xi \sigma_{\varepsilon}\left(X_{t-\varepsilon}\right)\left(W_{t}-W_{t-\varepsilon}\right)\right) \mid \mathcal{F}_{t-\varepsilon}\right] \\
& =\exp \left(\sqrt{-1} \xi\left\{X_{t-\varepsilon}+\int_{t-\varepsilon}^{t} b_{\varepsilon}\left(s, X_{t-\varepsilon}\right) \mathrm{d} s\right\}\right) \exp \left(-\frac{\varepsilon \sigma_{\varepsilon}\left(X_{t-\varepsilon}\right)^{2} \xi^{2}}{2}\right),
\end{aligned}
$$

thus, we obtain

$$
\left|\mathbb{E}\left[e^{\sqrt{-1} \xi X_{t}^{(\varepsilon)}} \mid \mathcal{F}_{t-\varepsilon}\right]\right|=\exp \left(-\frac{\varepsilon \sigma_{\varepsilon}\left(X_{t-\varepsilon}\right)^{2} \xi^{2}}{2}\right)
$$

Therefore, it holds that

$$
\begin{aligned}
& \left|\mathbb{E}\left[e^{\sqrt{-1} \xi X_{t}^{(\varepsilon)}} f_{\delta}\left(\left|\sigma_{\varepsilon}\left(X_{t-\varepsilon}\right)\right|\right)\right]\right| \leq \mathbb{E}\left[\left|\mathbb{E}\left[e^{\sqrt{-1} \xi X_{t}^{(\varepsilon)}} f_{\delta}\left(\left|\sigma_{\varepsilon}\left(X_{t-\varepsilon}\right)\right|\right) \mid \mathcal{F}_{t-\varepsilon}\right]\right|\right] \\
& =\mathbb{E}\left[f_{\delta}\left(\left|\sigma_{\varepsilon}\left(X_{t-\varepsilon}\right)\right|\right)\left|\mathbb{E}\left[e^{\sqrt{-1} \xi X_{t}^{(\varepsilon)}} \mid \mathcal{F}_{t-\varepsilon}\right]\right|\right]=\mathbb{E}\left[f_{\delta}\left(\left|\sigma_{\varepsilon}\left(X_{t-\varepsilon}\right)\right|\right) \exp \left(-\frac{\varepsilon \sigma_{\varepsilon}\left(X_{t-\varepsilon}\right)^{2} \xi^{2}}{2}\right)\right] \\
& =\mathbb{E}\left[f_{\delta}\left(\left|\sigma_{\varepsilon}\left(X_{t-\varepsilon}\right)\right|\right) \exp \left(-\frac{\varepsilon \sigma_{\varepsilon}\left(X_{t-\varepsilon}\right)^{2} \xi^{2}}{2}\right) \mathbf{1}_{\left\{\left|\sigma_{\varepsilon}\left(X_{t-\varepsilon}\right)\right| \geq \delta\right\}}\right] \leq \exp \left(-\frac{\varepsilon \delta^{2} \xi^{2}}{2}\right) .
\end{aligned}
$$

From Lemma 4.8, there exists $\bar{C}_{1}>0$ such that

$$
|\xi| \mathbb{E}\left[\left|X_{t}-X_{t}^{(\varepsilon)}\right|\right] \leq|\xi| \mathbb{E}\left[\left|X_{t}-X_{t}^{(\varepsilon)}\right|^{2}\right]^{1 / 2} \leq \bar{C}_{1}|\xi| \varepsilon .
$$

By using Schwarz's inequality, (57) and Lemma 4.3, there exists $\bar{C}_{2}>0$ such that

$$
\mathbb{E}\left[\left|\sigma\left(X_{t}\right)-\sigma\left(X_{t-\varepsilon}\right)\right|\right] \leq K_{0} \mathbb{E}\left[\left(1+\left|X_{t}\right|^{2 \ell}+\left|X_{t-\varepsilon}\right|^{2 \ell}\right)\right]^{1 / 2} \mathbb{E}\left[\left|X_{t}-X_{t-\varepsilon}\right|^{2}\right]^{1 / 2} \leq \bar{C}_{2} \varepsilon^{1 / 2} .
$$

By using Jensen's inequality, (63) and Lemma 4.4, there exists $\bar{C}_{3}>0$ such that

$$
\mathbb{E}\left[\left|\sigma\left(X_{t-\varepsilon}\right)-\sigma_{\varepsilon}\left(X_{t-\varepsilon}\right)\right|\right] \leq K_{0} \varepsilon^{1 / 2} \mathbb{E}\left[\left(1+\left|X_{t-\varepsilon}\right|^{\ell+2}\right)\left|X_{t-\varepsilon}\right|^{\ell}\right]^{1 / 2} \leq \bar{C}_{3} \varepsilon^{1 / 2} .
$$

From (67), 668), (69), (70) and (771) we obtain

$$
\left|\widehat{\mu}_{\delta, X_{t}}(\xi)\right| \leq \exp \left(-\frac{\varepsilon \delta^{2} \xi^{2}}{2}\right)+\bar{C}_{1}|\xi| \varepsilon+\left(\bar{C}_{2}+\bar{C}_{3}\right) \varepsilon^{1 / 2} .
$$


For $|\xi| \geq 2$, we choose $\varepsilon=(\log |\xi| /|\xi|)^{2} \in(0,1)$, then

$$
\left|\widehat{\mu}_{\delta, X_{t}}(\xi)\right| \leq \exp \left(-\frac{\delta^{2}(\log |\xi|)^{2}}{2}\right)+\bar{C}_{1} \frac{(\log |\xi|)^{2}}{|\xi|}+\left(\bar{C}_{2}+\bar{C}_{3}\right) \frac{\log |\xi|}{|\xi|},
$$

and thus

$$
\int_{\mathbb{R}}\left|\widehat{\mu}_{\delta, X_{t}}(\xi)\right|^{2} \mathrm{~d} \xi \leq 4+3 \int_{|\xi| \geq 2}\left\{\exp \left(-\delta^{2}(\log |\xi|)^{2}\right)+\frac{\bar{C}_{1}^{2}(\log |\xi|)^{4}}{|\xi|^{2}}+\frac{\left(\bar{C}_{2}+\bar{C}_{3}\right)^{2}(\log |\xi|)^{2}}{|\xi|^{2}}\right\} \mathrm{d} \xi<\infty
$$

which concludes the proof.

\section{Appendix}

\subsection{On some Beta type integral}

Lemma 5.1. Let $b>-1$ and $a \in[0,1)$. Then for any $t_{0}>0$,

$$
\int_{0}^{t_{0}} \mathrm{~d} t_{1} \cdots \int_{0}^{t_{m-1}} \mathrm{~d} t_{m} t_{m}^{b} \prod_{j=0}^{m-1}\left(t_{j}-t_{j+1}\right)^{-a}=\frac{t_{0}^{b+m(1-a)} \Gamma^{m}(1-a) \Gamma(1+b)}{\Gamma(1+b+m(1-a))} .
$$

Proof. Let $b>-1$ and $a \in[0,1)$. Using the change of variables $s=u t$, we have

$$
\int_{0}^{t} s^{b}(t-s)^{-a} \mathrm{~d} s=t^{b+1-a} \int_{0}^{1} u^{b}(1-u)^{-a} \mathrm{~d} u=t^{b+1-a} B(1+b, 1-a),
$$

where $B(x, y)=\int_{0}^{1} t^{x-1}(1-t)^{y-1}$ is the standard Beta function. Using this repeatedly, we obtain the statement.

\section{Acknowledgements}

The authors would like to thank an anonymous referee for his/her careful readings and comments. The first author was supported by JSPS KAKENHI Grant Number 17H06833 and 19K14552. The second author was supported by Sumitomo Mitsui Banking Corporation.

\section{References}

[1] Andersson, P. and Kohatsu-Higa, A. Unbiased simulation of stochastic differential equations using parametrix expansions. Bernoulli 23(3), 2028-2057, (2017).

[2] Aronson, D. G. Bounds for the fundamental solution of a parabolic equation. Bull. Amer. Math. Soc. 73, 890-896, (1967). 
[3] Bally, V. and Caramellino, L. Convergence and regularity of probability laws by using an interpolation method. Ann. Probab. 45(2), 1110-1159, (2017).

[4] Bally, V. and Kohatsu-Higa, A. A probabilistic interpretation of the parametrix method. Ann. Appl. Probab. 25(6), 3095-3138, (2015).

[5] Bally, V. and Talay, D. The law of the Euler scheme for stochastic differential equations: I. Convergence rate of the distribution function. Probab. Theory Relat. Fields. 104, 43-60, (1996).

[6] Baños, D. and Krühner, P. Optimal density bounds for marginals of Itô processes. Commun. Stoch. Anal. 10(2), 131-150, (2016).

[7] Baños, D. and Krühner, P. Hölder continuous densities of solutions of SDEs with measurable and path dependent drift coefficients. Stochastic Process. Appl. 127, 1785-1799, (2017).

[8] Bao, J. and Shao, J. Weak convergence of path-dependent SDEs with irregular coefficients. arXiv:1809.03088.

[9] Bass, R. F. Diffusions and elliptic operators. Springer Science and Business Media (1998).

[10] De Marco, S. Smoothness and asymptotic estimates of densities for SDEs with locally smooth coefficients and applications to square root-type diffusions. Ann. Appl. Probab., 21(4), 12821321, (2011).

[11] Deck, T. and Kruse, S. Parabolic differential equations with unbounded coefficients - a generalization of the parametrix method. Acta Appl. Math. 74, 71-91, (2002).

[12] Doumbia, M., Oudjane, N. and Warin, X. Unbiased Monte Carlo estimate of stochastic differential equations expectations. ESAIM Probab. Stat. 21, 56-87, (2017).

[13] Fabes, E. B. and Kenig, C. Examples of singular parabolic measures and singular transition probability densities. Duke Math. J. 48(4), 845-855, (1981).

[14] Fournier, N. and Printems, J. Absolute continuity of some one-dimensional processes. Bernoulli 16, 343-360, (2010).

[15] Friedman, A. Partial Differential Equations of Parabolic Type. Dover Publications Inc., (1964).

[16] Friedman, A. Stochastic differential equations and applications. Courier Corporation. Academic Press, New York, (1975).

[17] Frikha, N. and Li, L. Weak uniqueness and density estimates for sdes with coefficients depending on some path-functionals. arXiv:1707.01295,

[18] Goard, J. and Mazur, M. Stochastic volatility models and the pricing of VIX options. Math. Finance 23(3), 439-458, (2013).

[19] Gobet, E. and Labart, C. Sharp estimates for the convergence of the density of the Euler scheme in small time. Elect. Comm. in Probab., 13, 352-363, (2008). 
[20] Guyon, J. Euler schemes and tempered distributions. Stochastic. Process. Appl. 116, 887-904, (2006).

[21] Hayashi, M., Kohatsu-Higa, A. and Yûki, G. Local Hölder continuity property of the densities of solutions of SDEs with singular coefficients. J. Theor. Probab. 26, 1117-1134, (2013).

[22] Hayashi, M., Kohatsu-Higa, A. and Yûki, G. Hölder continuity property of the densities of SDEs with singular drift coefficients. Electorn. J. Probab. 19(77), 1-22, (2014).

[23] Henry-Labordere, P., Tan, X. and Touzi, N. Unbiased simulation of stochastic differential equations. Ann. Appl. Probab., 27(6), 3305-3341, (2017)

[24] Hutzenthalerm M., Jentzen, A. and Kloeden, P. E. Strong and weak divergence in finite time of Euler's method for stochastic differential equations with non-globally Lipschitz continuous coefficients. Proc. R. Soc. A 467, 1563-1576, (2011).

[25] Hutzenthalerm M., Jentzen, A. and Kloeden, P. E. Strong convergence of an explicit numerical method for SDEs with non-globally Lipschitz continuous coefficients. Ann. Appl. Probab., 22(4), 1611-1641, (2012).

[26] Ida, Y. and Imamura, Y. Towards the exact simulation using hyperbolic Brownian motion. Jpn. J. Ind. Appl. Math. 34(3), 833-843, (2017).

[27] Ikeda, N. and Watanabe, S. Stochastic differential equations and diffusion processes, second ed. volume 24 of North-Holland Mathematical Library, North-Holland Publishing Co., Amsterdam-New York; Kodansha, Ltd., Tokyo, (1981).

[28] Karatzas, I. and Shreve, S. E. Brownian motion and stochastic calculus. Second edition. Springer (1991).

[29] Kim, P. and Song, S. Two-sided estimates on the density of Brownian motion with singular drift. Illinois J. Math. 50(3), 635-688, (2006).

[30] Kloeden, P. and Platen, E. Numerical Solution of Stochastic Differential Equations. Springer (1995).

[31] Kohatsu-Higa, A., Taguchi, T. and Zhong, J. The Parametrix Method for Skew Diffusions. Potential Anal. 45, 299-329, (2016).

[32] Kohatsu-Higa, A. and Tanaka, A. A Malliavin calculus method to study densities of additive functionals of SDE's with irregular drifts. Ann. Inst. Henri Poincaré 48(3), 871-883, (2012).

[33] Kohatsu-Higa, A., Lejay, A. and Yasuda, K. On Weak Approximation of Stochastic Differential Equations with Discontinuous Drift Coefficient. J. Comput. Appl. Math., 326, 138-158, (2017).

[34] Kohatsu-Higa, A. and Yûki, G. Stochastic formulations of the parametrix method. to appear in ESAIM Probab. Stat.

[35] Konakov, V. and Mammen, E. Edgeworth type expansions for Euler schemes for stochastic differential equations. Monte Carlo Methods Appl. 8(3), 271-285, (2002). 
[36] Konakov, V., Kozhina, A. and Menozzi, S. Stability of densities for perturbed Diffusions and Markov Chains. ESAIM Probab. Stat. 21, 88-112, (2017).

[37] Kusuoka, S. and Stroock, D. W. Applications of the Malliavin calculus, I. Taniguchi Symposium on Stochastic Analysis, Katata 1982 (K. Itô editor) 271-306, North-Holland, Amsterdam (1983).

[38] Kusuoka, S. Continuity and Gaussian two-sided bounds of the density functions of the solutions to path-dependent stochastic differential equations via perturbation. Stochastic Process. Appl., 127, 359-384, (2017).

[39] Lemaire, V. and Menozzi, S. On some Non-Asymptotic Bounds for the Euler Scheme. Electron J. Probab. 15, 1645-1681, (2010).

[40] Leobacher, G. and Szölgyenyi, M. Convergence of the Euler-Maruyama method for multidimensional SDEs with discontinuous drift and degenerate diffusion coefficient. Numer. Math. 138(1), 219-239, (2018).

[41] Mackevičius, V. On the convergence rate of Euler scheme for SDE with Lipschitz drift and constant diffusion. Acta Appl. Math. 78, 301-310, (2003).

[42] Makhlouf, A. Existence and Gaussian bounds for the density of Brownian motion with random drift. Commun. Stoch. Anal. 10(2), 151-162, (2016).

[43] Maruyama, G. On the transition probability functions of the Markov process. Nat. Sci. Rep. Ochanomizu Univ. 5, 10-20, (1954).

[44] Maruyama, G. Continuous Markov processes and stochastic equations. Rend. Circ. Matem. Palermo 10, 48-90, (1955).

[45] McKean, H. P. and Singer, I. M. Curvature and the eigenvalues of the Laplacian. J. Differential Geom., 1, 43-69, (1967).

[46] Menoukeu Pamen, O. and Taguchi, D. Strong rate of convergence for the Euler-Maruyama approximation of SDEs with Hölder continuous drift coefficient. hastic Process. Appl. 127, $2542-2559,(2017)$.

[47] Mikulevicius, R. and Platen, E. Rate of convergence of the Euler approximation for diffusion processes. Math. Nachr., 151, 233-239, (1991).

[48] Nash, J. Continuity of solutions of parabolic and elliptec equations. Amer. J. Math. 80, 931954, (1958).

[49] Ngo, H-L. and Taguchi, D. Strong rate of convergence for the Euler-Maruyama approximation of stochastic differential equations with irregular coefficients. Math. Comp. 85(300), 17931819, (2016).

[50] Ngo, H-L. and Taguchi, D. Approximation for non-smooth functionals of stochastic differential equations with irregular drift. J. Math. Anal. Appl. 457(1), 361-388, (2018).

[51] Nualart, D. The Mallivain Calculus and Related Topics. Springer, (2006). 
[52] Olivera, C. and Tudor, C. A. Density for solutions to stochastic differential equations with unbounded drift. arXiv:1805.0671,

[53] Portenko, N. I. Generalized diffusion processes. Naukova Dumka, Kiev, 1982, English transl.: American Mathematical Society, Providence, R.I., (1990).

[54] Qian, Z. and Zheng, W. Sharp bounds for transition probability densities of a class of diffusions. C. R. Acad. Sci. Paris, Ser 335(11), 953-957, (2002).

[55] Qian, Z. and Zheng, W. Comparison theorem and estimates for transition probability densities of diffusion processes. Probab. Theory Relat. Fields. 127, 388-406, (2003).

[56] Qian, Z. and Zheng, W. A representation formula for transition probability densities of diffusions and applications. Stoch. Anal. Appl. 111, 57-76, (2004).

[57] Romito, M. A simple method for the existence of a density for stochastic evolutions with rough coefficients. Electorn. J. Probab. 23(113), 1-43, (2018).

[58] Sabanis, S. A note on tamed Euler approximations. Electron. Commun. Probab. 18(47), 1-10, (2013).

[59] Sabanis, S. Euler approximations with varying coefficients: the case of superlinearly growing diffusion coefficients. Ann. Appl. Probab., 25(4), 2083-2105, (2016).

[60] Sheu, S. J. Some estimates of the transition density of a nondegenerate diffusion Markov process. Ann. Probab., 19(2), 538-561, (1991).

[61] Shigekawa, I. Stochastic analysis, volume 224 of Translations of Mathematical Monographs. American Mathematical Society, Providence, RI, 2004. Translated from the 1998 Japanese original by the author, Iwanami Series in Modern Mathematics.

[62] Stroock, D. W. and Varadhan, S. R. S. Diffusion processes with continuous coefficients, I,II. Communications in Pure and Applied Mathematics, 22, 345-400, 479-530, (1969).

[63] Takeuchi, A. Joint distributions for stochastic functional differential equations. Stochastics 88(5), 711-736, (2016).

[64] Talay, D. and Tubaro, L. Expansion of the global error for numerical schemes solving stochastic differential equations. Stochastic Anal. Appl., 8, 94-120, (1990).

[65] Veretennikov, A. YU. On strong solution and explicit formulas for solutions of stochastic integral equations. Math. USSR Sb. 39, 387-403, (1981).

[66] Yan, B. L. The Euler scheme with irregular coefficients. Ann. Probab. 30(3), 1172-1194, (2002).

[67] Zvonkin, A. K. A transformation of the phase space of a diffusion process that removes the drift. Math. USSR Sbornik, 22, 129-148, (1974). 